\title{
Effect of branching on the delayed fluorescence and phosphorescence of simple borylated aryl amines
}

\author{
Sudhakar Pagidi, Neena K. Kalluvettukuzhy and Pakkirisamy Thilagar* \\ Department of Inorganic and Physical Chemistry, Indian Institute of Science, Bangalore - 560012, India \\ Ph. No. +91-080-2293 3353 Fax: 0091-80-23601552,*E-mail: thilagar@iisc.ac.in
}




\section{Experimental Section}

\section{Materials and methods}

All the chemicals were purchased from commercial suppliers and used as such unless otherwise mentioned. All reactions were carried under an atmosphere of purified Nitrogen using standard schlenk line techniques. THF was dried over sodium and distilled out under argon atmosphere.

The $400 \mathrm{MHz}{ }^{1} \mathrm{H}$ NMR, $100 \mathrm{MHz}{ }^{13} \mathrm{C}$ NMR were recorded on a Bruker Advance $400 \mathrm{MHz}$ NMR spectrometer. Solution ${ }^{1} \mathrm{H}$ NMR and ${ }^{13} \mathrm{C}$ NMR spectra were referenced internally to the solvent signals. Chemical shift multiplicities of ${ }^{1} \mathrm{H}$ NMR spectra are reported as singlet (s), broad singlet (brs), doublet $(\mathrm{d})$, triplet $(\mathrm{t})$, quartet $(\mathrm{q})$, and multiplet $(\mathrm{m}) .{ }^{11} \mathrm{~B}$ NMR spectra were recorded at $25^{\circ} \mathrm{C}$ on a JEOL $500 \mathrm{MHz}$ NMR Spectrometer operating at a frequency of 160.5 $\mathrm{MHz}$ for ${ }^{11} \mathrm{~B}$. ${ }^{11} \mathrm{~B}$ NMR chemical shift values were referenced to the external standard boron signal of $\mathrm{BF}_{3} \cdot \mathrm{Et}_{2} \mathrm{O}$. High resolution mass spectra (HRMS) were recorded on a Micromass Q-ToF High Resolution Mass Spectrometer by electrospray ionization (ESI) method.

Solutions of all the compounds for spectral measurements were prepared using spectrophotometric grade solvents, microbalance $( \pm 0.1 \mathrm{mg}$ precision $)$ and standard volumetric glass wares. Quartz cuvettes with sealing screw caps were used for the solution state spectral measurements. Electronic absorption spectra were recorded on a Perkin Elmer LAMBDA 750 UV/visible spectrophotometer. Luminescence measurements were performed using an Edinburgh Instruments FLS980 spectrometer equipped with a double monochromator for both excitation and emission, operating in right-angle geometry mode and the highly sensitive photomultiplier tube (RED PMT in Cooled Housing) positioned after a double emission monochromator. All spectra were fully corrected for the spectral response of the instrument. Temperature dependent ( $77 \mathrm{~K}$ to $298 \mathrm{~K}$ ) measurements were performed using an Oxford Instruments OPTISTAT DN2 cryostat controlled by an Oxford Instruments Mercury iTC temperature controller connected to the FLS980 spectrometer. Samples were allowed to equilibrate at each temperature before measurements were conducted. Time gated emission spectra were excitation source was a pulsed microsecond flash lamp $(\mu \mathrm{F} 1)$ with a pulse width of $1.1 \mu \mathrm{S}$. In delayed emission and decay measurements, the species having shorter excited state lifetime will be discarded by the time gated circuit attached to PMT detector, depending on the 
delay time. Fluorescence lifetimes were recorded using the time-correlated single photon counting (TCSPC) method using an Edinburgh Instruments FLS980 spectrometer described above. Measurements were made in right-angle geometry mode, and the emission was collected through a polarizer set to the magic angle. Solutions were excited by a pulsed LED at $330 \mathrm{~nm}$, at repetition rates of $10 \mathrm{MHz}$, as appropriate. The microsecond lifetimes were measured using the FLS980 spectrometer described above for steady-state measurements. The excitation source was a pulsed microsecond flash lamp $(\mu \mathrm{F} 1)$ with a pulse width of $1.1 \mu \mathrm{S}$. The quality of all decay fits was judged to be satisfactory, based on the calculated values of the reduced $\chi^{2}$ and Durbin Watson parameters and visual inspection of the weighted residuals. Absolute solid state quantum yields were measured using calibrated Horiba JOBIN YVON Integrating sphere. Solution state quantum yields were calculated with reference to anthracence $\left(\phi=0.27\right.$ in $\mathrm{EtOH}$ at $\left.27{ }^{\circ} \mathrm{C}\right)$ as standard. ${ }^{1}$

Single-crystal X-ray diffraction (SCXRD) studies were carried out with a Bruker SMART APEX diffractometer equipped with 4-axis goniometer. The data were integrated using SAINT, and an empirical absorption correction was applied with SADABS. ${ }^{2}$ The structures were solved by direct methods and refined by full matrix least-squares on $\mathrm{F}^{2}$ using SHELXTL software.

Density functional theory (DFT) calculations were done using B3LYP functional with 6$31 \mathrm{G}(\mathrm{d})$ basis set as incorporated in Gaussian 09 package for all the atoms, mixing the exact Hartree-Fock-type exchange with Becke's exchange functional and that proposed by Lee-YangParr for the correlation contribution. ${ }^{3}$ The molecular structures obtained from SCXRD measurements were taken as the input for the calculations. The optimized structures and the frontier molecular orbitals (FMOs) were viewed using Gaussview5.0. 


\section{Synthesis}
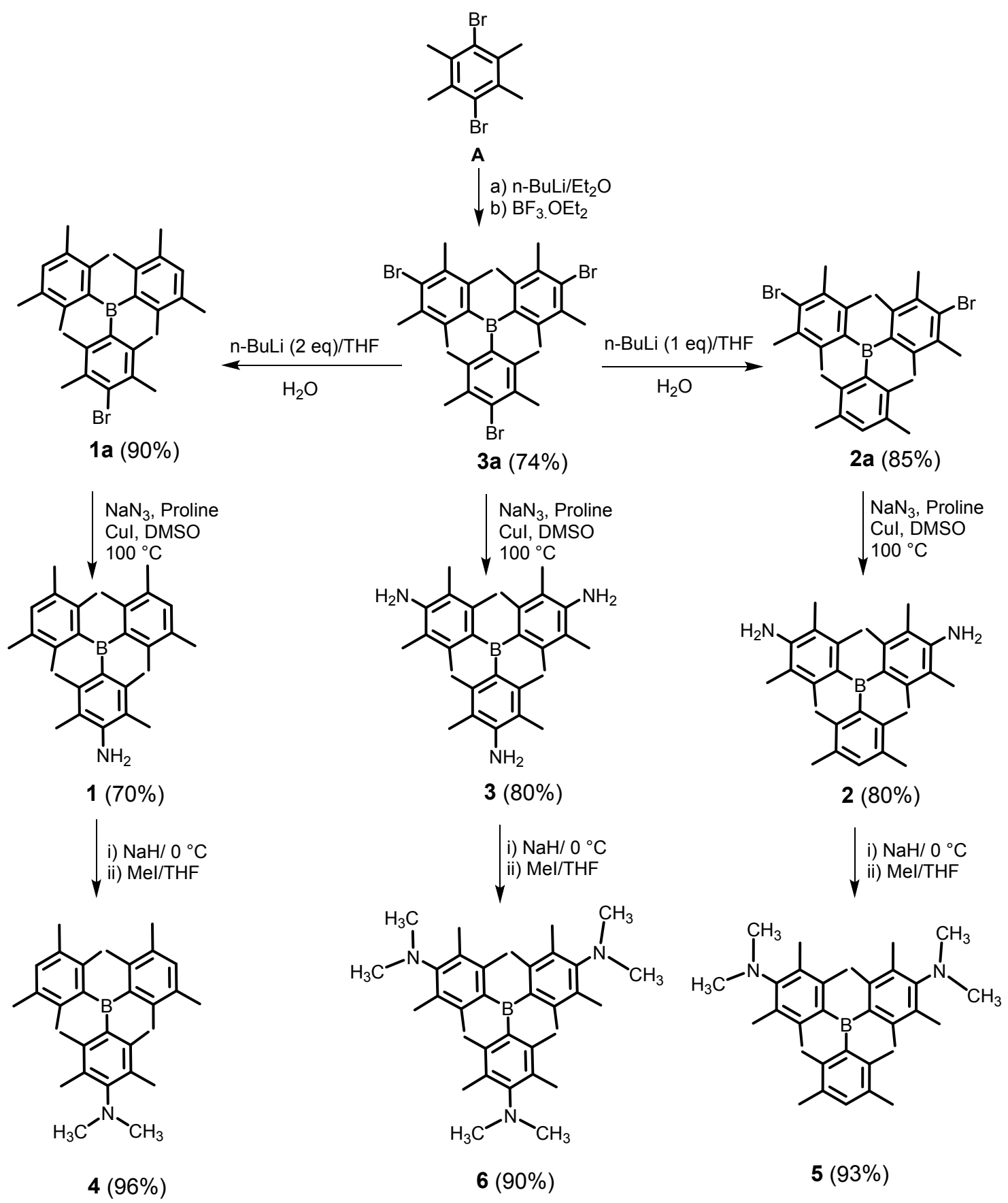

Scheme S1. Synthesis of compounds 1-6 (the yields are shown in parenthesis). 
1,4-dibromo-2,3,5,6-tetramethylbenzene (A). ${ }^{4}$ Iodine $(0.1 \mathrm{~g}, 0.4 \mathrm{mmol})$ was added to dichloromethane $(25 \mathrm{~mL})$ solution of 1,2,4,5-tetramethylbenzene $(2.5 \mathrm{~g}, 18 \mathrm{mmol})$ followed by the addition of a solution of $\mathrm{Br}_{2}(2.4 \mathrm{~mL}, 7.5 \mathrm{~g}, 47 \mathrm{mmol})$ in $20 \mathrm{~mL}$ of dichloromethane dropwise under stirring. The reaction mixture was heated under reflux for 1.5 hours and cooled to room temperature. $5 \mathrm{M}$ aq. $\mathrm{NaOH}(50 \mathrm{~mL})$ was added. The product was collected by filtration, washed with $\mathrm{H}_{2} \mathrm{O}$ and dried and recrystallized from dichloromethane to furnish 1,4-dibromo2,3,5,6-tetramethylbenzene. Yield: 93\%. ${ }^{1} \mathrm{H} \mathrm{NMR}\left(400 \mathrm{MHz}^{\mathrm{CDCl}}{ }_{3}, 25{ }^{\circ} \mathrm{C}\right): \delta(\mathrm{ppm}) 2.48 .{ }^{13} \mathrm{C}$ NMR (100 MHz $\left.\mathrm{CDCl}_{3}, 25^{\circ} \mathrm{C}\right): \delta(\mathrm{ppm}) 135.2,128.3,22.4$.

Tris(4-bromo-2,3,5,6-tetramethylphenyl)borane (3a). ${ }^{4}$ To a solution of 1,4-dibromo-2,3,5,6tetramethylbenzene (A) $(14.1 \mathrm{~g}, 48.3 \mathrm{mmol})$ in dry $\mathrm{Et}_{2} \mathrm{O}(400 \mathrm{~mL})$ was added dropwise hexane solution of $n-\mathrm{BuLi}(1.6 \mathrm{M}, 30 \mathrm{~mL}, 48.0 \mathrm{mmol})$ at $-78^{\circ} \mathrm{C}$. The reaction mixture was allowed to warm to $0{ }^{\circ} \mathrm{C}$ and stirred for $20 \mathrm{~min}$. To the reaction mixture was added $\mathrm{BF}_{3} . \mathrm{OEt}_{2}(2.0 \mathrm{~mL}, 15.8$ mmol) at $-78{ }^{\circ} \mathrm{C}$. The reaction mixture was warmed to room temperature over $1 \mathrm{~h}$ and stirring continued for $16 \mathrm{~h}$. The reaction was quenched with water and organics was extracted with $\mathrm{Et}_{2} \mathrm{O}$. The combined organic extract was washed with brine, dried over anhydrous $\mathrm{Na}_{2} \mathrm{SO}_{4}$ and concentrated under reduced pressure to give a colorless solid. The crude product was suspended in a mixture of $\mathrm{Et}_{2} \mathrm{O} / \mathrm{MeOH}$ in 1:1 ratio. The obtained precipitate was collected and washed with $\mathrm{MeOH}$ to give 3a $(7.62 \mathrm{~g}, 11.8 \mathrm{mmol})$ in $74 \%$ yield as a white solid: M.P. $292{ }^{\circ} \mathrm{C} .{ }^{1} \mathrm{H}$ NMR $(400$ $\left.\mathrm{MHz} \mathrm{CDCl}_{3}, 25^{\circ} \mathrm{C}\right): \delta(\mathrm{ppm}) 2.34(\mathrm{~s}, 18 \mathrm{H}), 1.99(\mathrm{~s}, 18 \mathrm{H}) .{ }^{13} \mathrm{C} \mathrm{NMR}\left(100 \mathrm{MHz} \mathrm{CDCl}_{3}, 25{ }^{\circ} \mathrm{C}\right): \delta$ (ppm) 147.9, 137.0, 133.8, 131.2, 21.4, 20.8.

(4-bromo-2,3,5,6-tetramethylphenyl)bis(2,3,5,6-tetramethylphenyl)borane (1a). $n$-BuLi (1.2 $\mathrm{mL}, 2.5 \mathrm{M}$ solution in hexanes, $3.4 \mathrm{mmol})$ was added drop wise to a THF (40 mL) solution of 3a ( $1 \mathrm{~g}, 1.5 \mathrm{mmol})$ at $-78{ }^{\circ} \mathrm{C}$ under stirring. After $1 \mathrm{~h}$ the reaction was quenched with water and warmed to room temperature over $3 \mathrm{~h}$. All volatiles were removed under reduced pressure and the crude product was purified by column chromatography over silica-gel with hexanes as eluent to obtain 1a as colourless solid. Yield: 90\%. ${ }^{1} \mathrm{H}$ NMR (400 MHz, $\left.\mathrm{CDCl}_{3}, 25{ }^{\circ} \mathrm{C}\right): \delta(\mathrm{ppm}) 6.93$ (s, 2H), 2.34 (s, 6H), 2.14 (s, 12H), 2.02 (s, 6H), 1.90 (s, 12H). ${ }^{13} \mathrm{C} \mathrm{NMR} \mathrm{(100} \mathrm{MHz,} \mathrm{CDCl} 3,25$ 
$\left.{ }^{\circ} \mathrm{C}\right): \delta(\mathrm{ppm}) 149.6,137.5,136.6,136.4,133.9,133.7,133.3,133.1,131.9,131.1,21.7,21.3$, 20.7, 19.6.

Bis(4-bromo-2,3,5,6-tetramethylphenyl)(2,3,5,6-tetramethylphenyl)borane (2a). To a solution of tris (4-bromo-2,3,5,6-tetramethylphenyl)borane ( $1 \mathrm{~g}, 1.5 \mathrm{mmol})$ in THF $(40 \mathrm{~mL})$ at $78{ }^{0} \mathrm{C}, n$-BuLi (2.5 M solution in hexanes, $1.5 \mathrm{mmol}$ ) was added drop wise under stirring conditions. The reaction was quenched with water after $1 \mathrm{~h}$ and the resultant reaction mixture was allowed to warm room temperature over $3 \mathrm{~h}$. All the volatiles were removed under reduced pressure and the product was purified by column chromatography over silica-gel with hexanes as eluent, to furnish a colourless solid. Yield: $85 \%$. ${ }^{1} \mathrm{H}$ NMR (400 MHz, $\mathrm{CDCl}_{3}, 25{ }^{\circ} \mathrm{C}$ ): $\delta$ (ppm) 6.94 (s, 1H), 2.34 (s, 12H), 2.14 (s, 6H), 2.01 (s, 12H), 1.89 (s, 6H). ${ }^{13} \mathrm{C}$ NMR (100 MHz, $\mathrm{CDCl}_{3}$, $\left.25^{\circ} \mathrm{C}\right): \delta$ (ppm) 148.6, 137.6, 137.4, 136.5, 134.1, 133.9, 133.6, 131.4, 21.7, 21.4, 20.7, 19.6.

Caution: The reaction with sodium azide is not recommended for higher scales.

4-(bis(2,3,5,6-tetramethylphenyl)boryl)-2,3,5,6-tetramethylaniline (1). A reaction flask containing $15 \mathrm{~mL}$ DMSO was charged with L-proline $(61 \mathrm{mg}, 0.5 \mathrm{mmol})$, sodium azide (58 mg, $0.9 \mathrm{mmol}), \mathrm{Cu}(\mathrm{I}) \mathrm{I}(77 \mathrm{mg}, 0.4 \mathrm{mmol})$ and $1 \mathrm{a}(200 \mathrm{mg}, 0.4 \mathrm{mmol})$. The reaction mixture was slowly heated to $100{ }^{\circ} \mathrm{C}$. During the course of the reaction, the solution color changed from colorless to dark green. The progress of the reaction was monitored by TLC. After consumption of all the starting materials; the mixture was passed through celite and the compound was purified by column chromatography over silica-gel using hexane-ethyl acetate $(1: 1)$ as eluent to obtain the title compound as pale green color solid. Yield: 70\%, M.P. 96-99 ${ }^{\circ} \mathrm{C} .{ }^{1} \mathrm{H}$ NMR (400 $\left.\mathrm{MHz} \mathrm{CDCl}_{3}, 25^{\circ} \mathrm{C}\right): \delta(\mathrm{ppm}) 6.87(\mathrm{~s}, 1 \mathrm{H}), 3.67(\mathrm{~s}, 4 \mathrm{H}), 2.12(\mathrm{~s}, 6 \mathrm{H}), 2.03(\mathrm{~s}, 12 \mathrm{H}), 1.93(\mathrm{~s}, 6 \mathrm{H})$, $1.90(\mathrm{~s}, 12 \mathrm{H}) ;{ }^{13} \mathrm{C} \mathrm{NMR}\left(100 \mathrm{MHz}, \mathrm{CDCl}_{3}, 25{ }^{\circ} \mathrm{C}\right): \delta(\mathrm{ppm}) 143.6,142.7,137.1,117.8,20.7$, 13.7. ${ }^{11} \mathrm{~B}$ NMR (376.5 MHz, $\left.\mathrm{CDCl}_{3}, 25{ }^{\circ} \mathrm{C}\right): \delta$ (ppm) 75.5. ESI-MS, (positive ion mode): calcd for $\mathrm{C}_{30} \mathrm{H}_{40} \mathrm{BN}$ 425.3524 Da, observed 426.3322 [M+H] $]^{+}$. Anal.Cald. for $\mathrm{C}_{30} \mathrm{H}_{40} \mathrm{BN}$ : C, 84.69; $\mathrm{H}$, 9.48; N, 3.29. Found C, 84.72; H, 9.46; N, 3.25.

4,4'-((2,3,5,6-tetramethylphenyl)boranediyl)bis(2,3,5,6-tetramethylaniline) (2). Compound 2 was prepared by following a similar procedure as that of $\mathbf{1}$. Quantities involved for preparation of 2 are as follows: L-proline (100 mg, $0.9 \mathrm{mmol})$, sodium azide (100 mg, $1.5 \mathrm{mmol}), \mathrm{Cu}(\mathrm{I}) \mathrm{I}$ (134 $\mathrm{mg}, 0.7 \mathrm{mmol})$ and $\mathbf{2 a}(200 \mathrm{mg}, 0.35 \mathrm{mmol})$. Appearance: yellow color solid. Yield: 80\%, M.P. 
196-198 ${ }^{\circ} \mathrm{C} .{ }^{1} \mathrm{H}$ NMR $\left(400 \mathrm{MHz} \mathrm{CDCl}_{3}, 25^{\circ} \mathrm{C}\right): \delta(\mathrm{ppm}) 6.87(\mathrm{~s}, 1 \mathrm{H}), 3.67(\mathrm{~s}, 4 \mathrm{H}), 2.12(\mathrm{~s}, 6 \mathrm{H})$, $2.03(\mathrm{~s}, 6 \mathrm{H}), 2.00(\mathrm{~s}, 6 \mathrm{H}), 1.93(\mathrm{~s}, 6 \mathrm{H}), 1.90(\mathrm{~s}, 6 \mathrm{H}) ;{ }^{13} \mathrm{C} \mathrm{NMR}\left(100 \mathrm{MHz}, \mathrm{CDCl}_{3}, 25{ }^{\circ} \mathrm{C}\right): \delta$ (ppm) 151.5, 143.6, 141.9, 137.2, 137.1, 136.5, 133.2, 132.1, 117.9, 20.7, 19.5, 13.7. ${ }^{11} \mathrm{~B}$ NMR (376.5 MHz, $\left.\mathrm{CDCl}_{3}, 25^{\circ} \mathrm{C}\right): \delta(\mathrm{ppm})$ 74.7. ESI-MS, (positive ion mode): calcd for $\mathrm{C}_{30} \mathrm{H}_{41} \mathrm{BN}_{2}$ 440.3363 Da, observed 441.3425 [M+H] $]^{+}$. Anal.Cald. for $\mathrm{C}_{30} \mathrm{H}_{41} \mathrm{BN}_{2} \mathrm{C}, 81.10 ; \mathrm{H}, 9.38 ; \mathrm{N}, 6.36$. Found C, 81.06; H, 9.35; N, 6.39.

4,4',4"-boranetriyltris(2,3,5,6-tetramethylaniline) (3). Compound 3 was prepared by following a similar procedure as that of $\mathbf{1}$. Quantities involved for preparation of $\mathbf{3}$ are as follows: L-proline (225 mg, $1.5 \mathrm{mmol}$ ), sodium azide (150 mg, $2.5 \mathrm{mmol}), \mathrm{Cu}(\mathrm{I}) \mathrm{I}$ (220 mg, 1.15 mmol) and 3a (250 mg, $0.38 \mathrm{mmol})$. Appearance: pale yellow color solid. Yield: 80\%, M.P. 244-246 ${ }^{\circ} \mathrm{C}$; ${ }^{1} \mathrm{H}$ NMR (400 $\left.\mathrm{MHz} \mathrm{CDCl}_{3}, 25{ }^{\circ} \mathrm{C}\right): \delta(\mathrm{ppm}) 3.64(\mathrm{~s}, 2 \mathrm{H}), 2.02$ (s, 6H), 1.95 (s, $12 \mathrm{H}) ;{ }^{13} \mathrm{C} \mathrm{NMR}\left(100 \mathrm{MHz}, \mathrm{CDCl}_{3}, 25{ }^{\circ} \mathrm{C}\right): \delta(\mathrm{ppm}) 143.2,142.7,137.1,117.8,20.7,13.7 .{ }^{11} \mathrm{~B}$ NMR (376.5 MHz, $\left.\mathrm{CDCl}_{3}, 25{ }^{\circ} \mathrm{C}\right): \delta$ (ppm) 74.4. ESI-MS, (positive ion mode): calcd for $\mathrm{C}_{30} \mathrm{H}_{42} \mathrm{BN}_{3}$ 455.3472 Da, observed 456.3550 Da [M+H] $]^{+}$. Anal.Cald. for $\mathrm{C}_{30} \mathrm{H}_{42} \mathrm{BN}_{3} \mathrm{C}, 79.11 ; \mathrm{H}$, 9.29; N, 9.23. Found C, 79.14; H, 9.26; N, 9.19.

4-(bis(2,3,5,6-tetramethylphenyl)boryl)- $N, N, 2,3,5,6$-hexamethylaniline (4). Sodium hydride (53 mg, $2.2 \mathrm{mmol}$ ) was added to THF solution of $1(425 \mathrm{mg}, 1 \mathrm{mmol})$ at $0{ }^{\circ} \mathrm{C}$ under stirring. After 30 minutes, $\mathrm{MeI}(140 \mu \mathrm{L}, 2.2 \mathrm{mmol})$ was added drop wise to the reaction mixture at $\sim<0$ ${ }^{\circ} \mathrm{C}$, the reaction mixture was warmed to room temperature and the stirring was continued for another $12 \mathrm{~h}$. The progress of the reaction was monitored by TLC. After complete consumption of reactants, the reaction mixture was quenched with saturated aqueous $\mathrm{NH}_{4} \mathrm{Cl}$ solution. The organic layer was extracted with ethyl acetate and dried over $\mathrm{Na}_{2} \mathrm{SO}_{4}$, and concentrated under reduced pressure. The crude product was purified by column chromatography over silica-gel using petroleum ether as eluent to obtain the title compound as colourless solid. Yield: 96\%. M.P. $98-113{ }^{\circ} \mathrm{C} .{ }^{1} \mathrm{H}$ NMR $\left(400 \mathrm{MHz} \mathrm{CDCl}_{3}, 25^{\circ} \mathrm{C}\right): \delta(\mathrm{ppm}) 6.90(\mathrm{~s}, 1 \mathrm{H}), 2.82(\mathrm{~d}, J=1.6 \mathrm{~Hz}$, $6 \mathrm{H}), 2.14(\mathrm{~d}, J=3.6 \mathrm{~Hz}, 12 \mathrm{H}), 2.08(\mathrm{~s}, 6 \mathrm{H}), 1.92(\mathrm{~d}, J=3.2 \mathrm{~Hz}, 12 \mathrm{H}), 1.89(\mathrm{~s}, 6 \mathrm{H}) ;{ }^{13} \mathrm{C}$ NMR $\left(100 \mathrm{MHz}, \mathrm{CDCl}_{3}, 25^{\circ} \mathrm{C}\right): \delta(\mathrm{ppm}) 151.1,150.2,137.2,136.5,136.4,133.4,132.9,132.8,43.4$, 20.7, 20.6, 19.5, 19.4, 15.7. ${ }^{11} \mathrm{~B} \operatorname{NMR}\left(376.5 \mathrm{MHz}, \mathrm{CDCl}_{3}, 25{ }^{\circ} \mathrm{C}\right): \delta(\mathrm{ppm})$ 77.6. ESI-MS, (positive ion mode): calcd for $\mathrm{C}_{32} \mathrm{H}_{44} \mathrm{BN} 453.3567$ Da, observed 454.3591 Da $[\mathrm{M}+\mathrm{H}]^{+}$. 
Anal.Cald. for $\mathrm{C}_{32} \mathrm{H}_{44} \mathrm{BN} \mathrm{C}, 84.75 ; \mathrm{H}, 9.78 ; \mathrm{N}, 3.09$. Found C, 84.72; H, 9.82; N, 3.12.

\section{4,4'-((2,3,5,6-tetramethylphenyl)boranediyl)bis $(N, N, 2,3,5,6$-hexamethylaniline $)$}

Compound 5 was prepared by following a similar procedure as that of 4. Quantities used for preparation of 5 are as follows: $\mathrm{NaH}(100 \mathrm{mg}, 4.4 \mathrm{mmol}), \mathbf{2}(440 \mathrm{mg}, 1 \mathrm{mmol})$ and $\mathrm{MeI}(270 \mu \mathrm{L}$, $4.4 \mathrm{mmol})$. Appearance: Dark yellow color solid. Yield: 93\%. M.P. 162-165 ${ }^{\circ} \mathrm{C} .{ }^{1} \mathrm{H}$ NMR (400 $\left.\mathrm{MHz} \mathrm{CDCl}_{3}, 25^{\circ} \mathrm{C}\right): \delta(\mathrm{ppm}) 6.89(\mathrm{~s}, 1 \mathrm{H}), 2.81(\mathrm{~s}, 12 \mathrm{H}), 2.13(\mathrm{~s}, 6 \mathrm{H}), 2.08(\mathrm{~d}, J=3.6 \mathrm{~Hz}, 12 \mathrm{H})$, $1.91(\mathrm{~s}, 6 \mathrm{H}), 1.89(\mathrm{~d}, J=3.2 \mathrm{~Hz}, 12 \mathrm{H}) ;{ }^{13} \mathrm{C} \mathrm{NMR}\left(100 \mathrm{MHz}, \mathrm{CDCl}_{3}, 25{ }^{\circ} \mathrm{C}\right): \delta(\mathrm{ppm}) 150.9$, 137.2, 137.1, 136.5, 133.3, 132.8, 132.7, 132.6, 43.4, 20.7, 20.6, 20.5, 19.4, 15.7, 15.6. ${ }^{11} \mathrm{~B}$ NMR (376.5 MHz, $\mathrm{CDCl}_{3}, 25^{\circ} \mathrm{C}$ ): $\delta$ (ppm) 76.21. ESI-MS, (positive ion mode): calcd for $\mathrm{C}_{34} \mathrm{H}_{49} \mathrm{BN}_{2}$ 496.3989 Da, observed 497.4005 Da $[\mathrm{M}+\mathrm{H}]^{+}$. Anal.Cald. for $\mathrm{C}_{34} \mathrm{H}_{49} \mathrm{BN}_{2} \mathrm{C}, 82.24 ; \mathrm{H}, 9.95 ; \mathrm{N}$, 5.64. Found C, 82.20; H, 9.92; N, 5.67.

4,4', $4^{\prime \prime}$-boranetriyltris( $N, N, 2,3,5,6$-hexamethylaniline) (6). Compound 6 was prepared by following a similar procedure as that of 4 . Quantities used for the preparation of 6 are as follows: $\mathrm{NaH}$ (160 mg, $6.6 \mathrm{mmol}), 3$ (455 mg, $1 \mathrm{mmol})$ and $\mathrm{MeI}(410 \mu \mathrm{L}, 6.6 \mathrm{mmol})$. Appearance: White color solid. Yield: 90\%. M.P. 256-260 ${ }^{\circ} \mathrm{C} .{ }^{1} \mathrm{H}$ NMR (400 $\left.\mathrm{MHz} \mathrm{CDCl}_{3}, 25{ }^{\circ} \mathrm{C}\right): \delta(\mathrm{ppm}) 2.81(\mathrm{~s}$, 18H), 2.07 (s, 18H), 1.89 (s, 18H); $\left.{ }^{13} \mathrm{C} \mathrm{NMR} \mathrm{(100} \mathrm{MHz,} \mathrm{CDCl}_{3}, 25{ }^{\circ} \mathrm{C}\right): \delta(\mathrm{ppm}) 150.8,147.5$, 137.2, 132.7, 43.4, 20.4, 15.6. ${ }^{11} \mathrm{~B}$ NMR (376.5 $\left.\mathrm{MHz} \mathrm{CDCl}_{3}, 25{ }^{\circ} \mathrm{C}\right): \delta(\mathrm{ppm})$ 75.9. ESI-MS, (positive ion mode): calcd for $\mathrm{C}_{36} \mathrm{H}_{54} \mathrm{BN}_{3} 539.4411 \mathrm{Da}$, observed $540.4575 \mathrm{Da}[\mathrm{M}+\mathrm{H}]^{+}$. Anal.Cald. for $\mathrm{C}_{36} \mathrm{H}_{54} \mathrm{BN}_{3}$ C, 80.12; H, 10.09; N, 7.79. Found: C, 80.10; H, 10.06; N, 7.81. 


\section{NMR Spectral characterization}

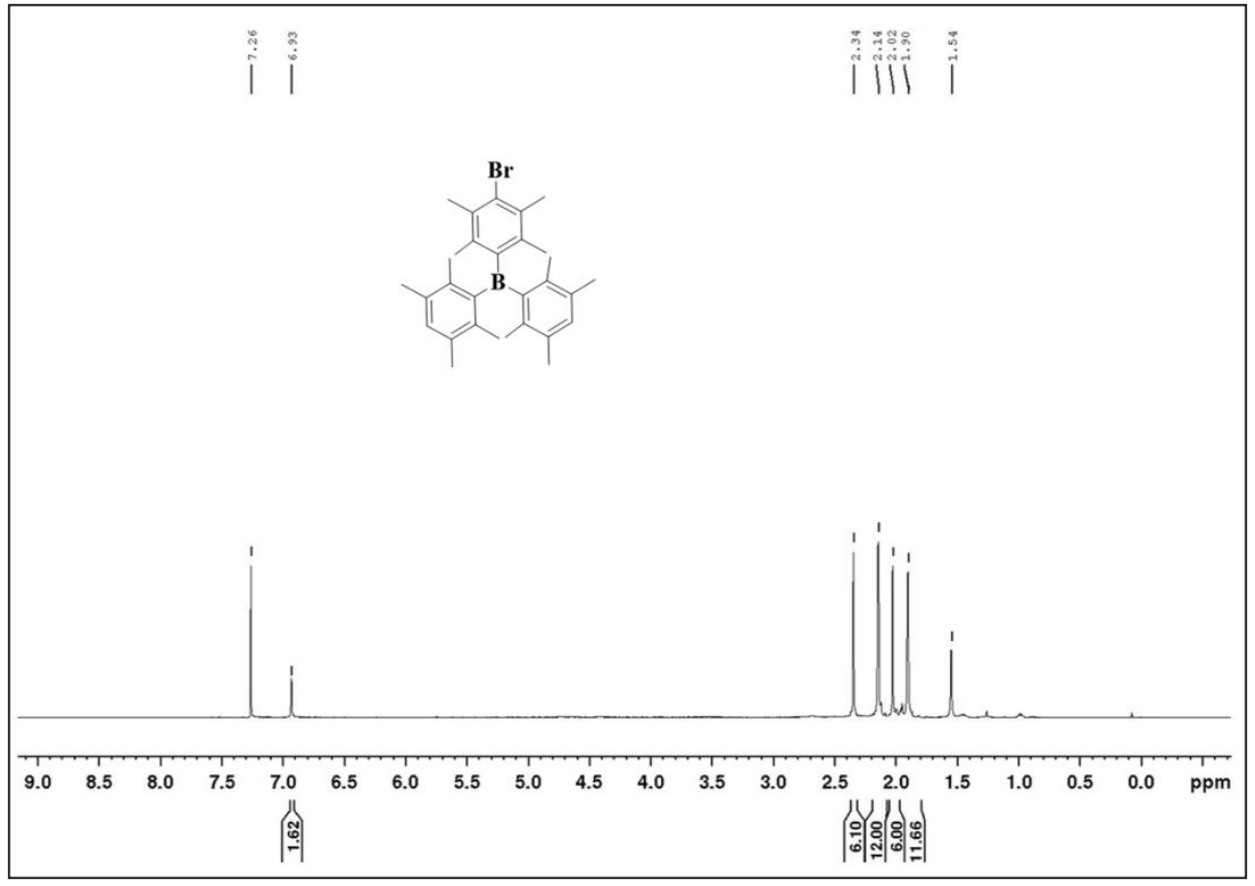

Figure S1. ${ }^{1} \mathrm{H}$ NMR spectrum of $\mathbf{1 a}$

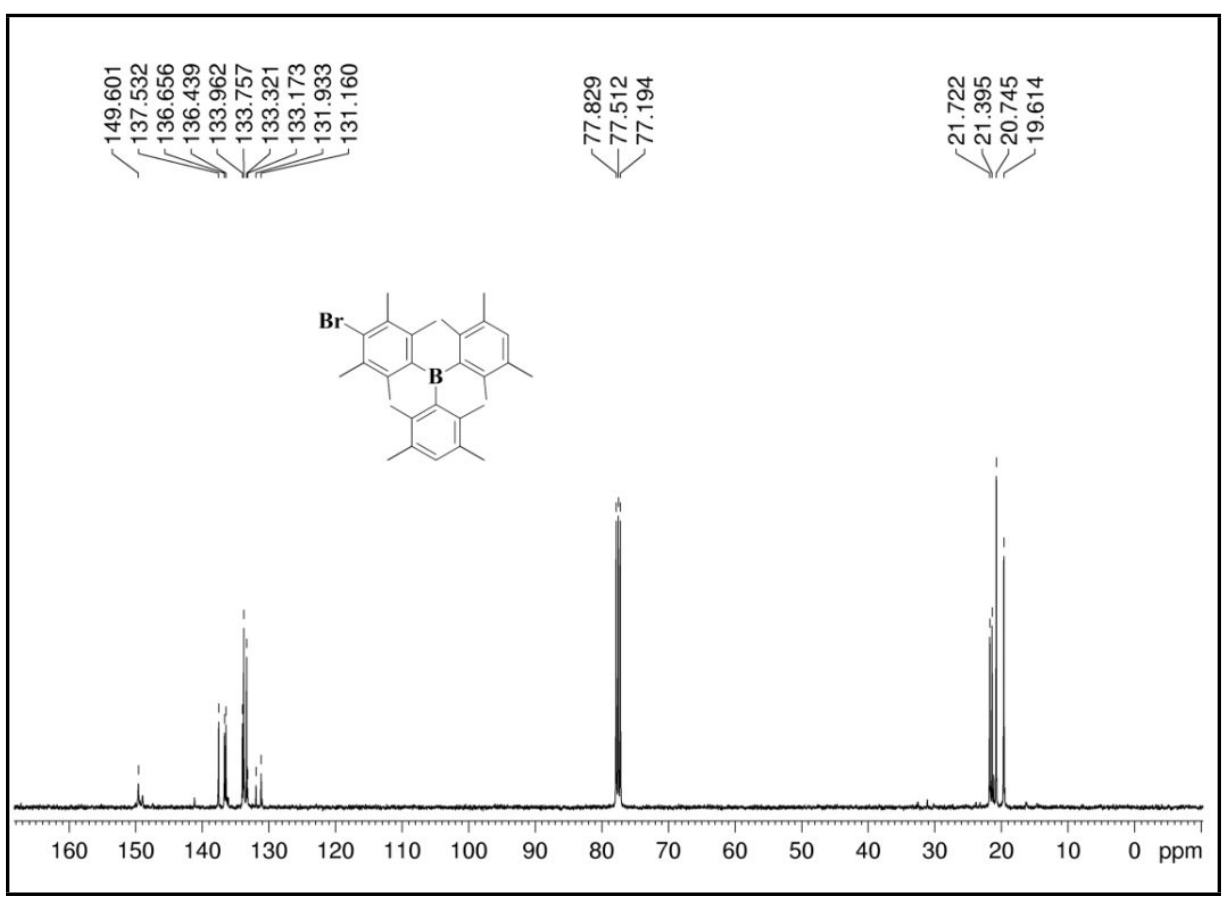

Figure S2. ${ }^{13} \mathrm{C}$ NMR spectrum of $\mathbf{1 a}$ 


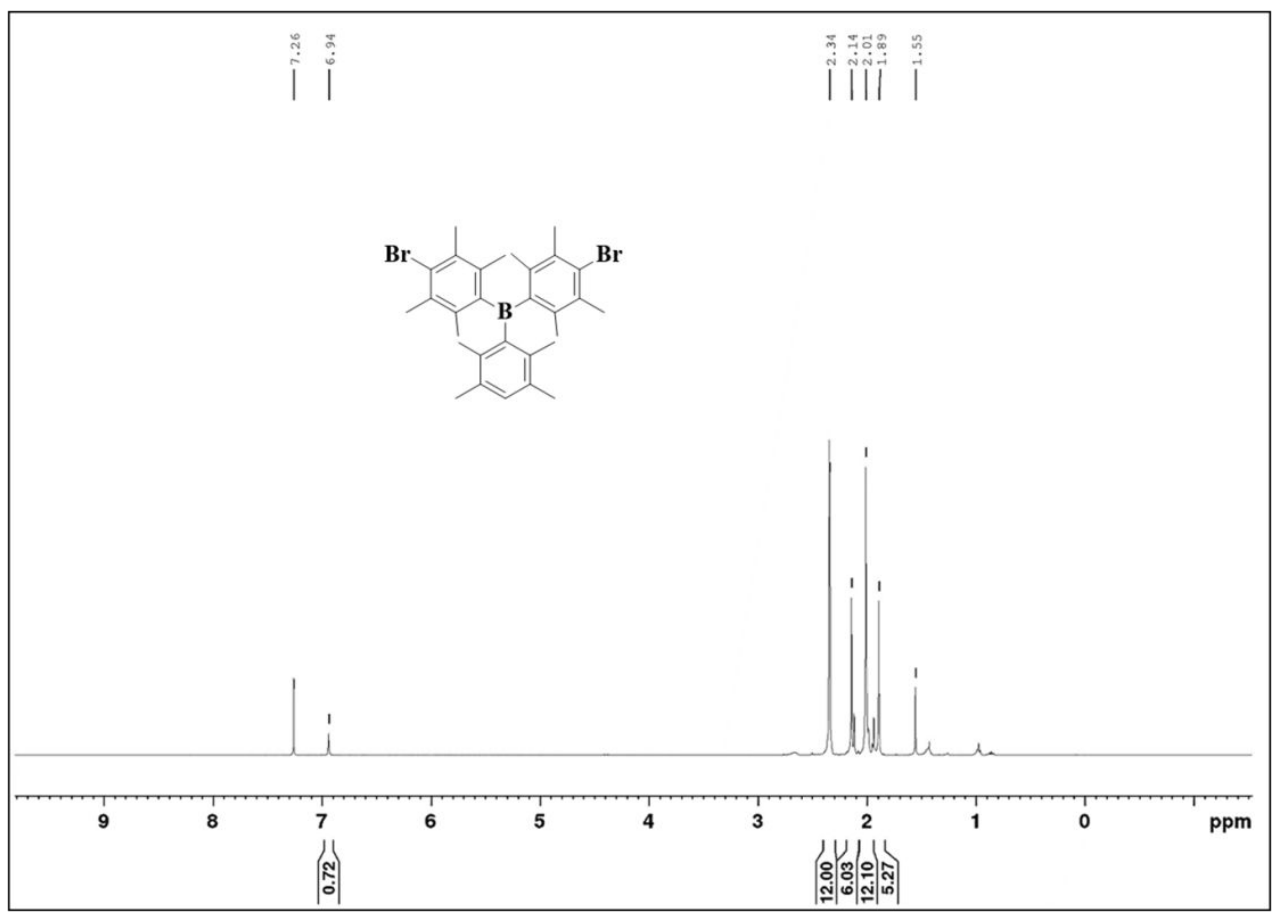

Figure S3. ${ }^{1} \mathrm{H}$ NMR spectrum of $\mathbf{2 a}$

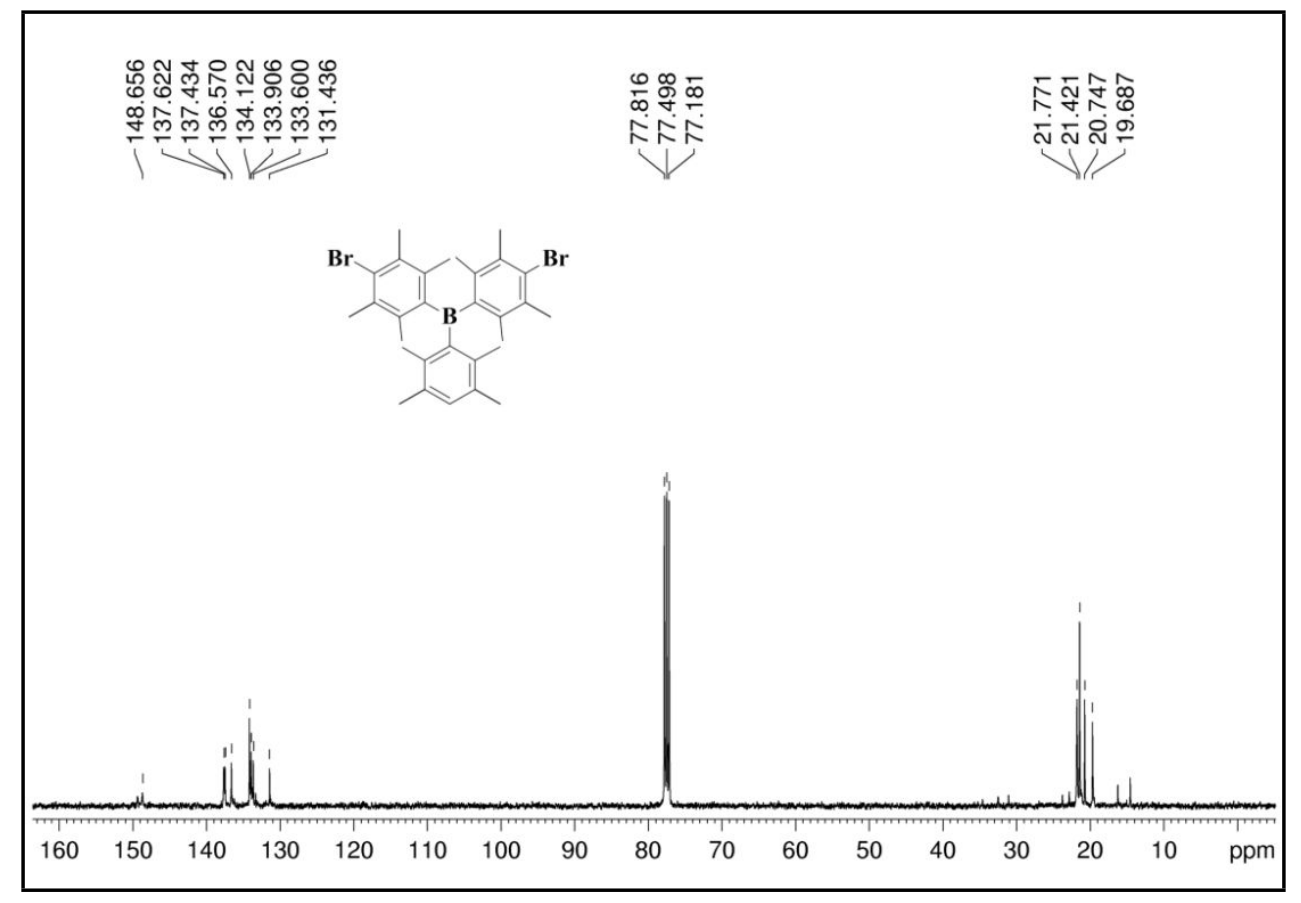

Figure $\mathbf{S 4} \cdot{ }^{13} \mathrm{C}$ NMR spectrum of $\mathbf{2 a}$ 


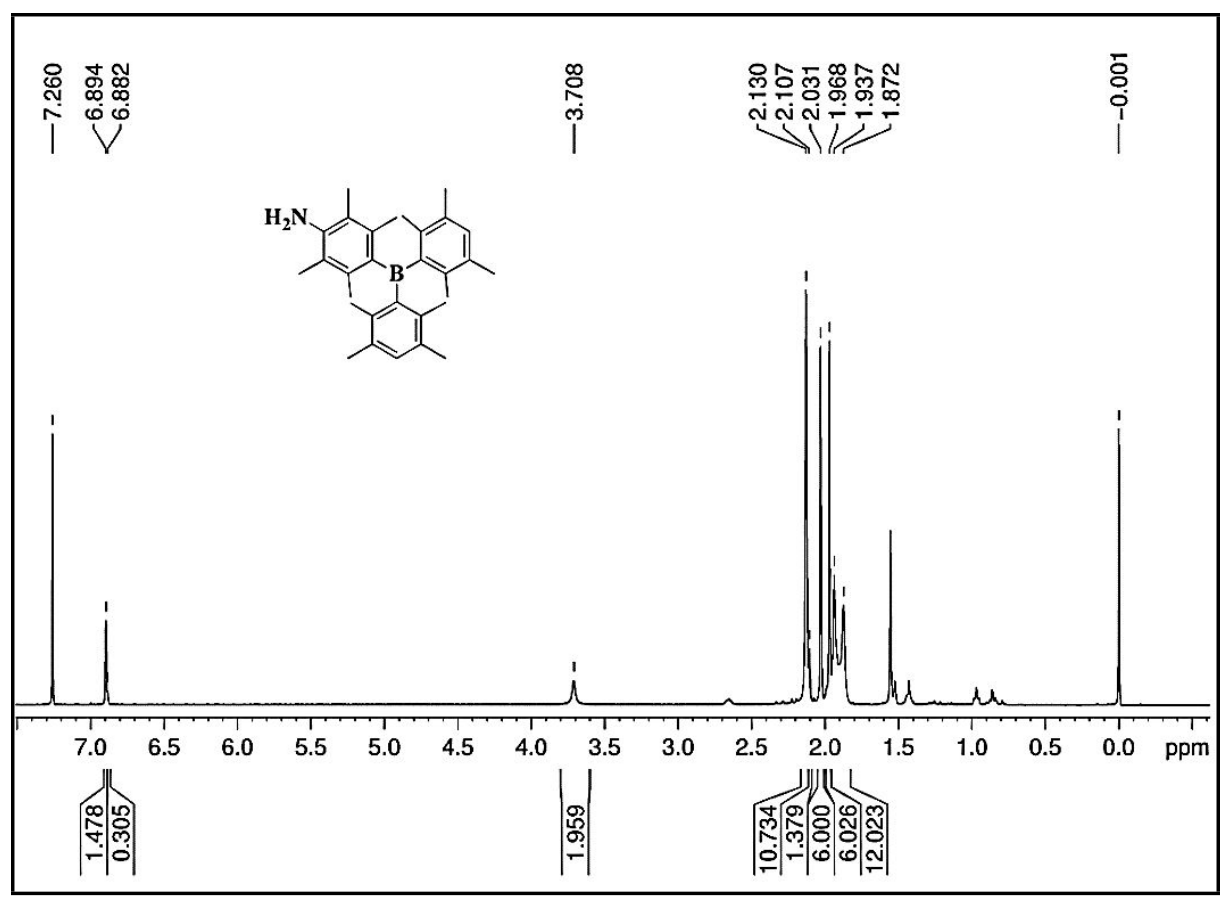

Figure S5. ${ }^{1} \mathrm{H}$ NMR spectrum of $\mathbf{1}$

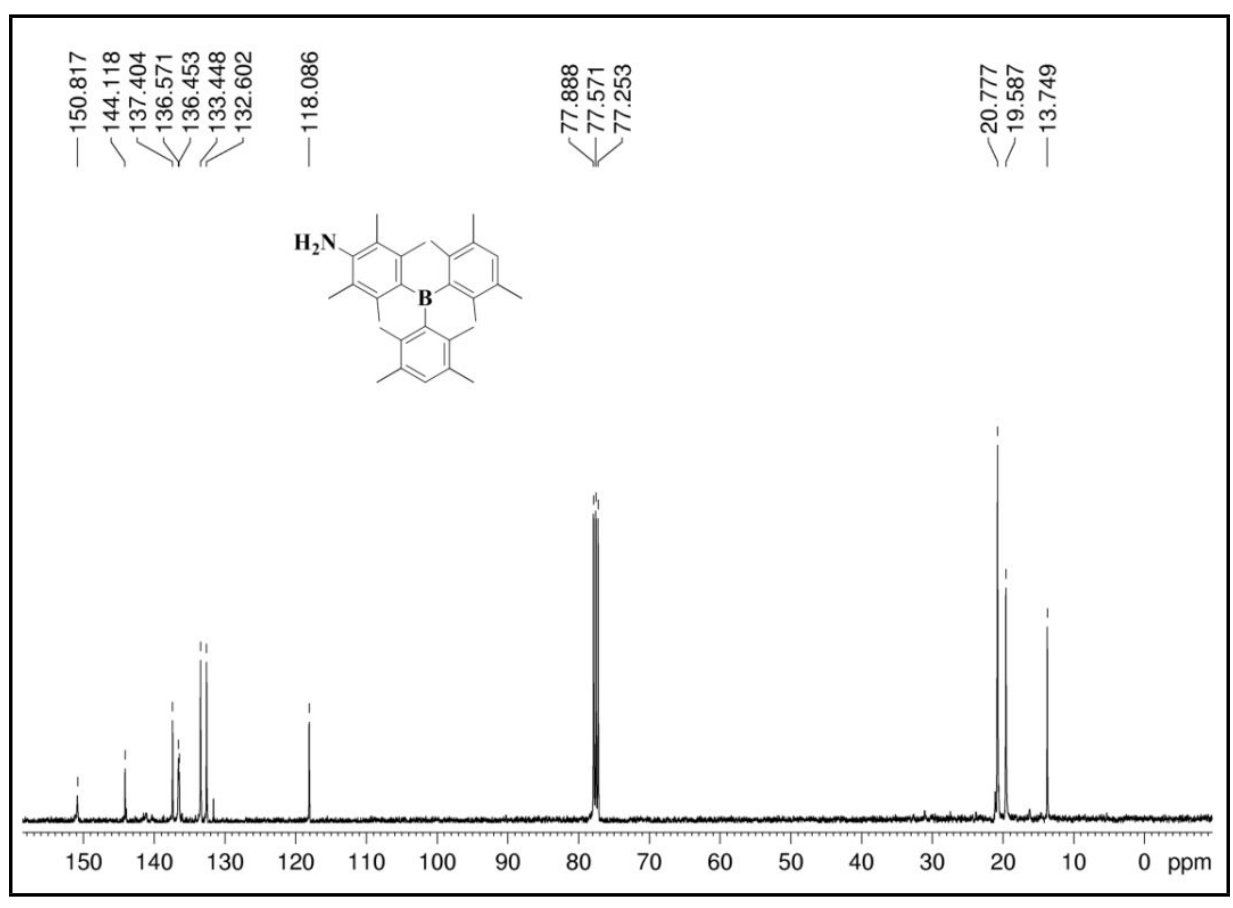

Figure S6. ${ }^{13} \mathrm{C}$ NMR spectrum of $\mathbf{1}$ 


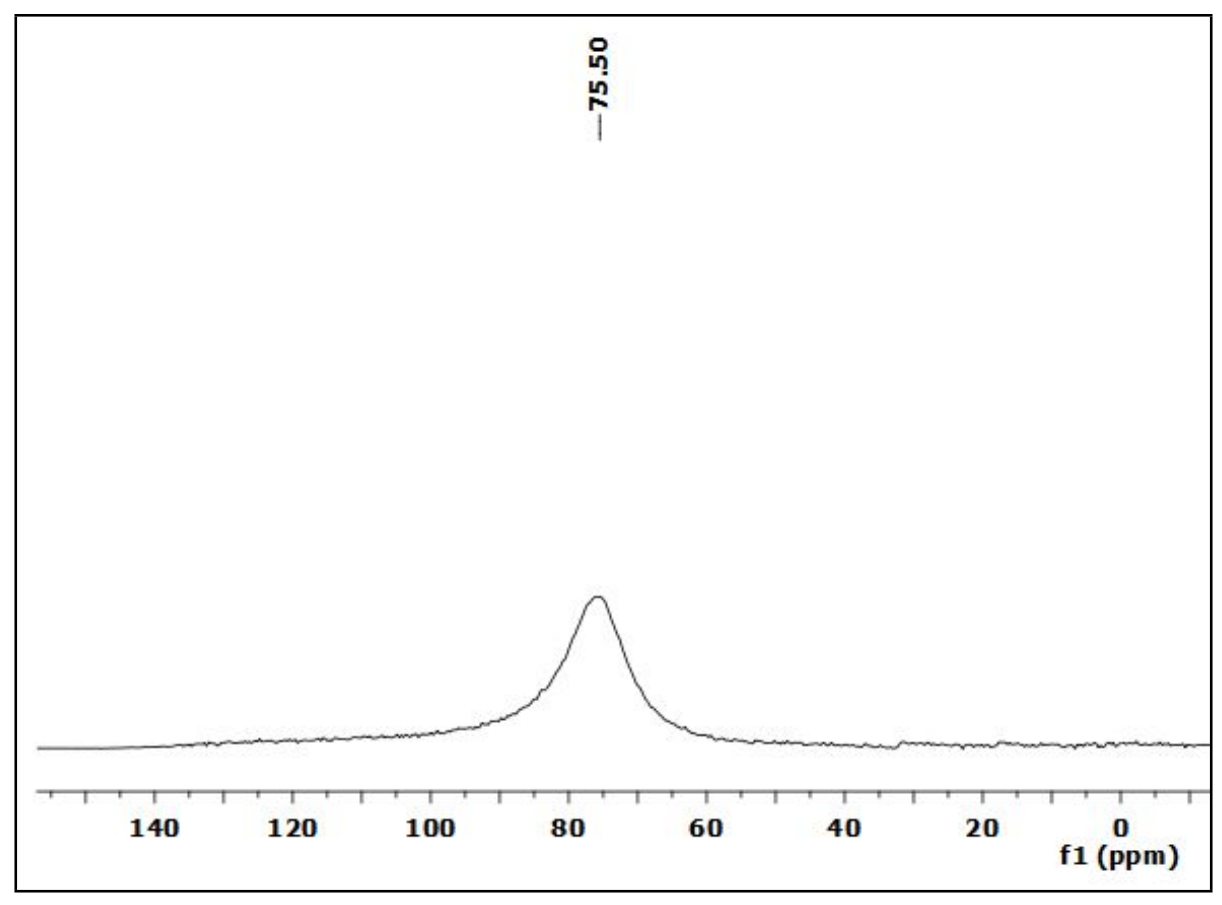

Figure S7. ${ }^{11}$ B NMR spectrum of 1

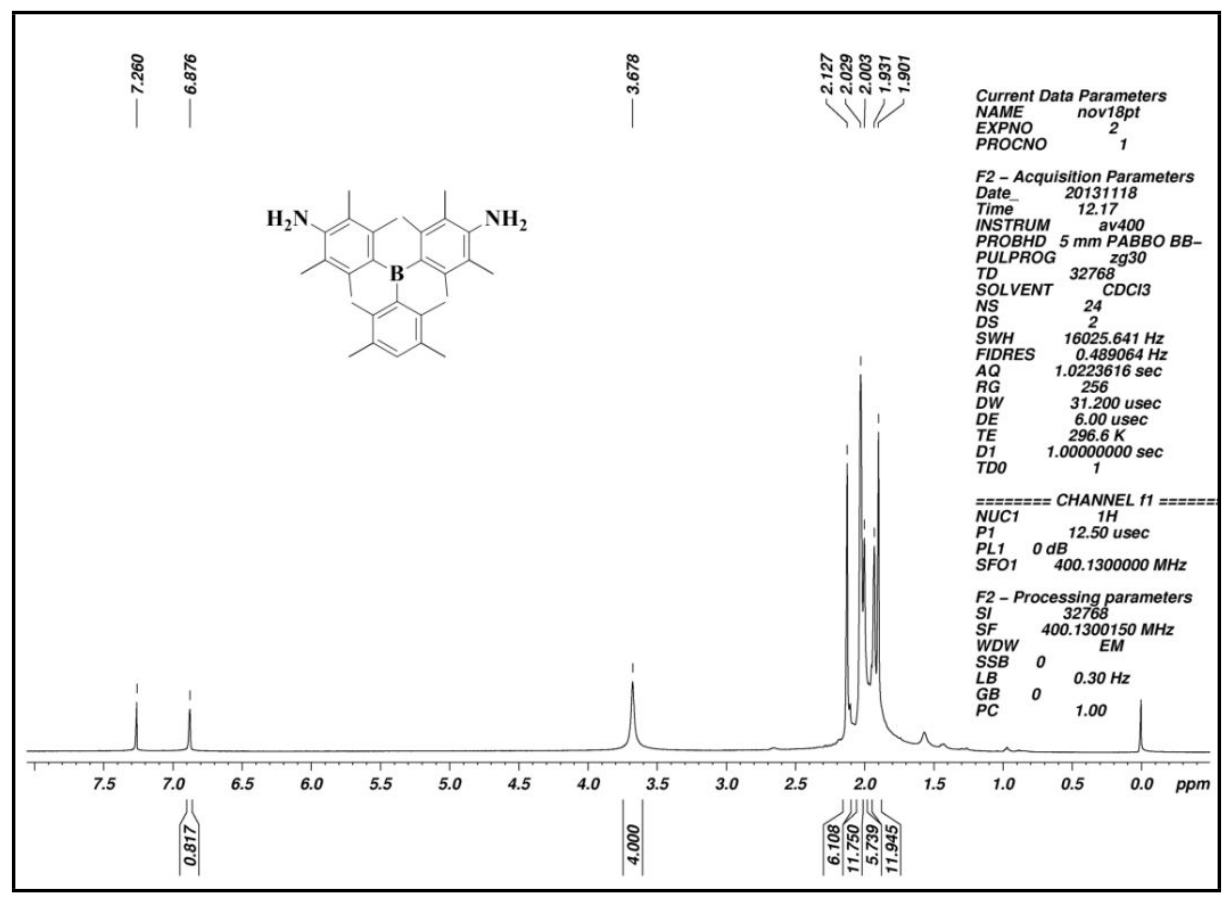

Figure S8. ${ }^{1} \mathrm{H}$ NMR spectrum of $\mathbf{2}$ 


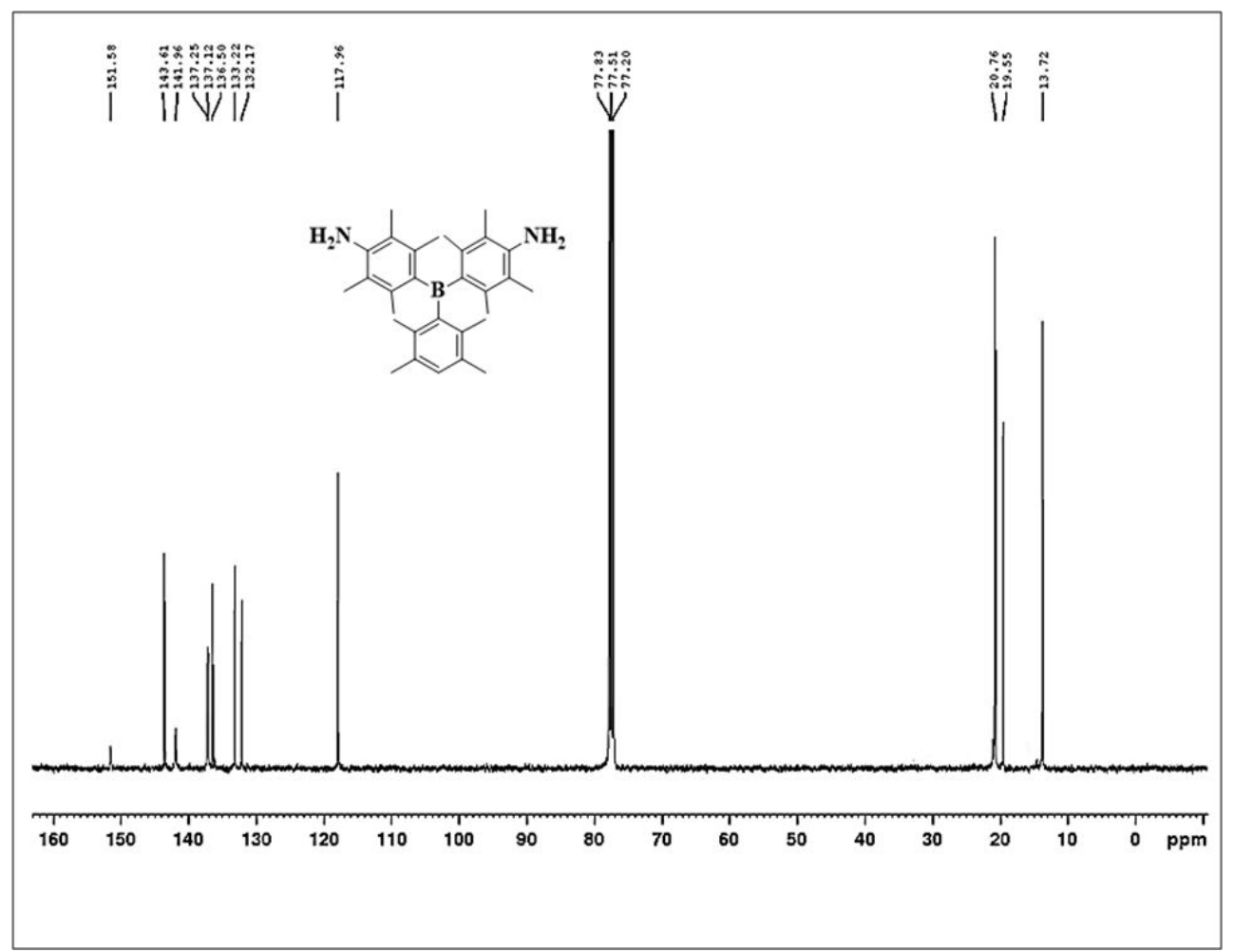

Figure S9. ${ }^{13} \mathrm{C}$ NMR spectrum of 2

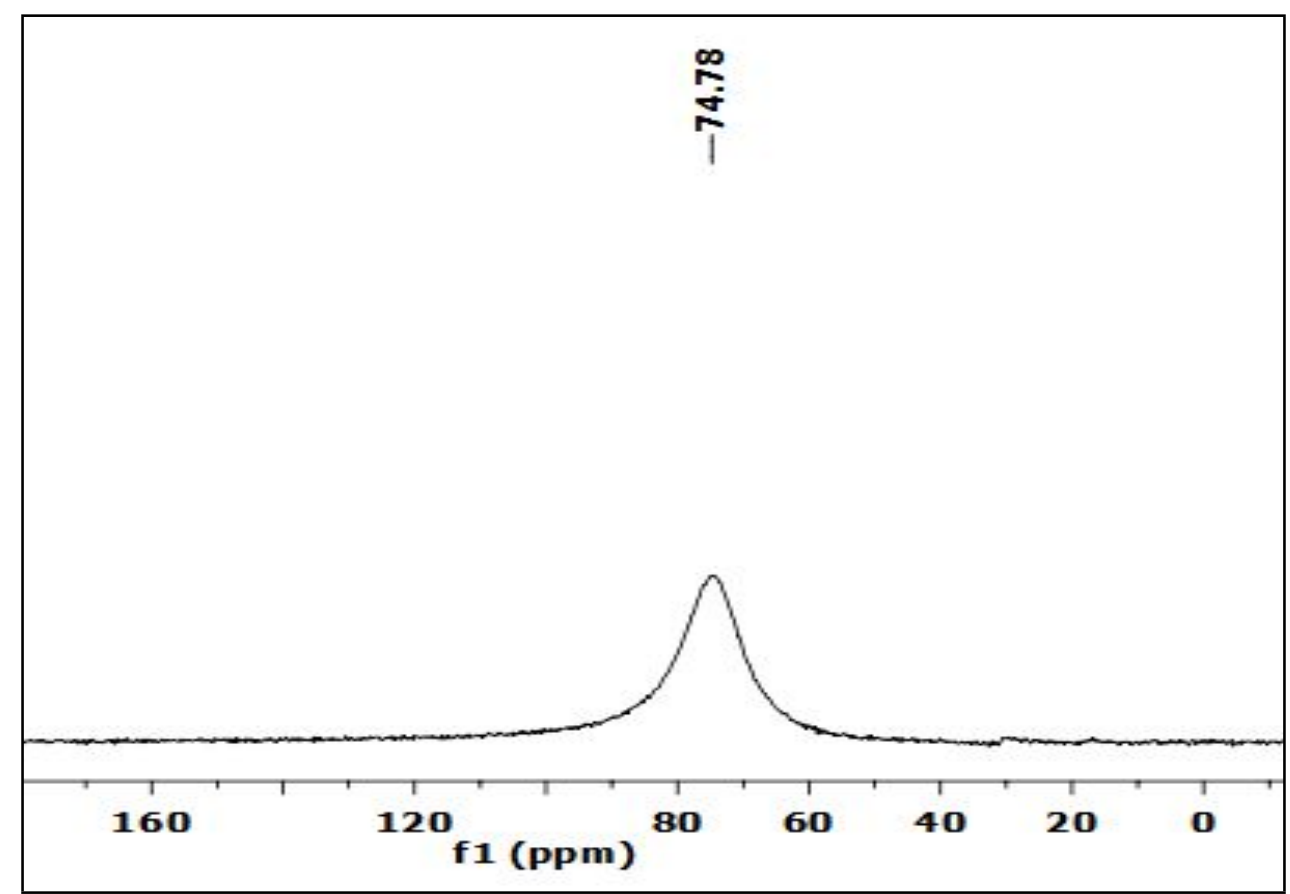

Figure S10. ${ }^{11} \mathrm{~B}$ NMR spectrum of 2 


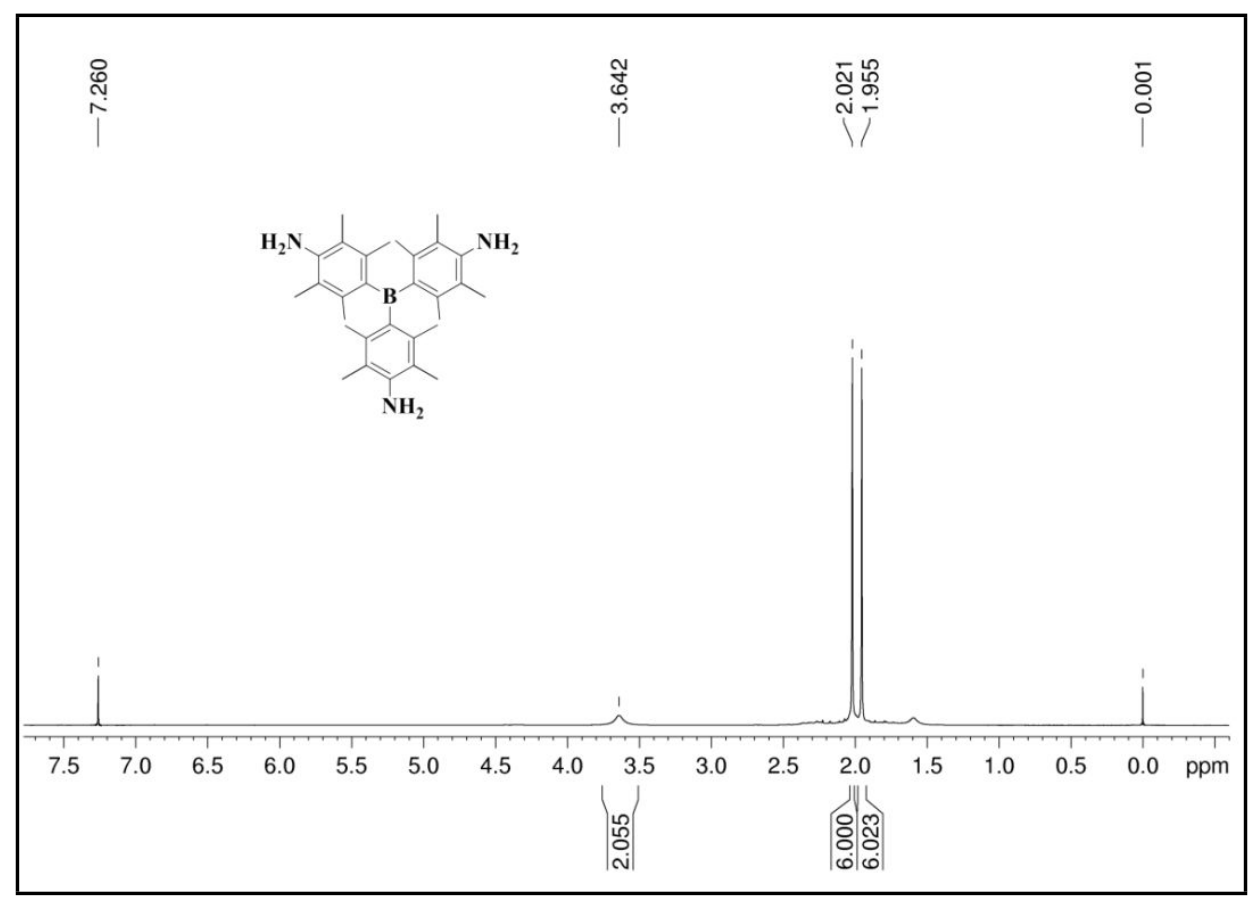

Figure S11. ${ }^{1} \mathrm{H}$ NMR spectrum of 3

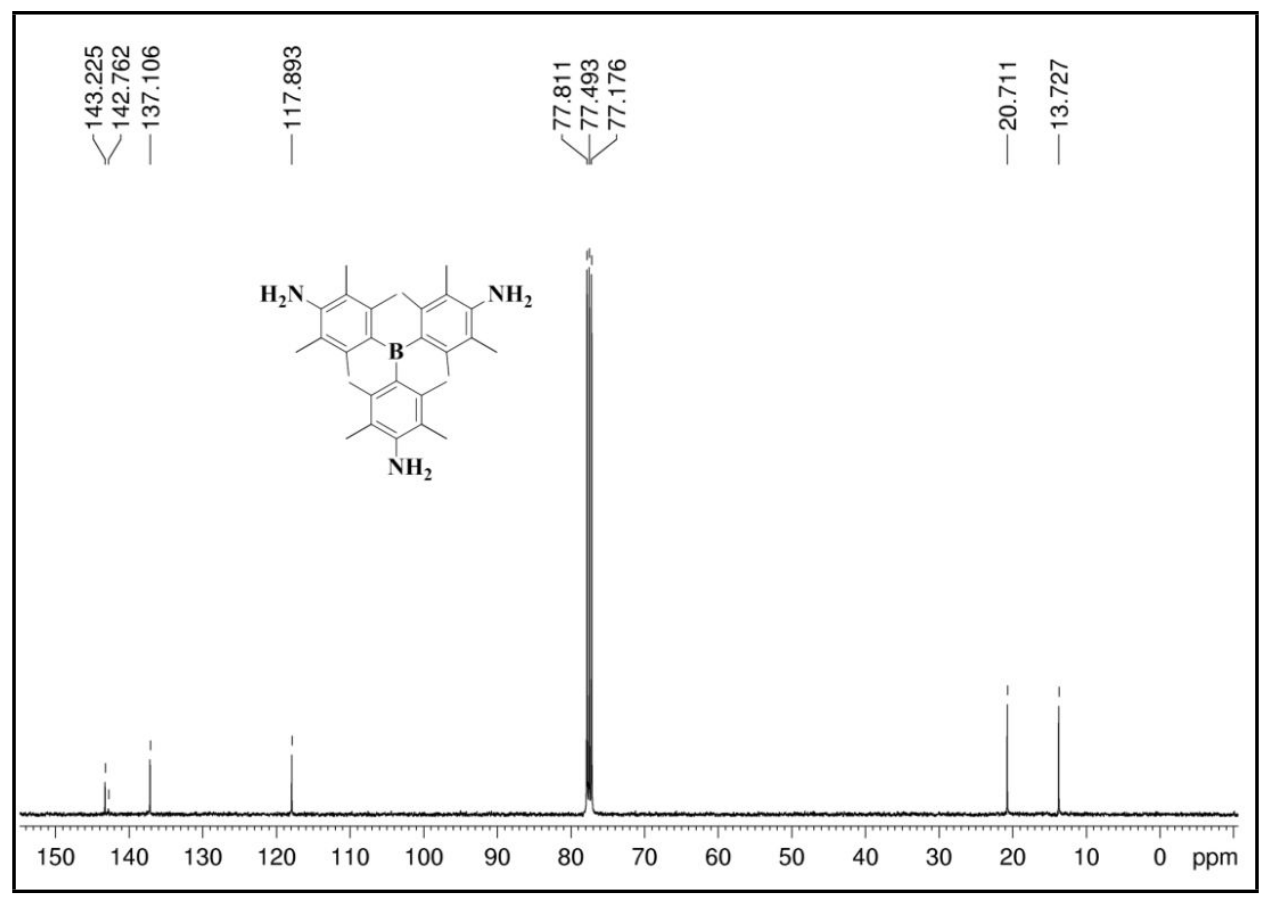

Figure S12. ${ }^{13} \mathrm{C}$ NMR spectrum of $\mathbf{3}$ 


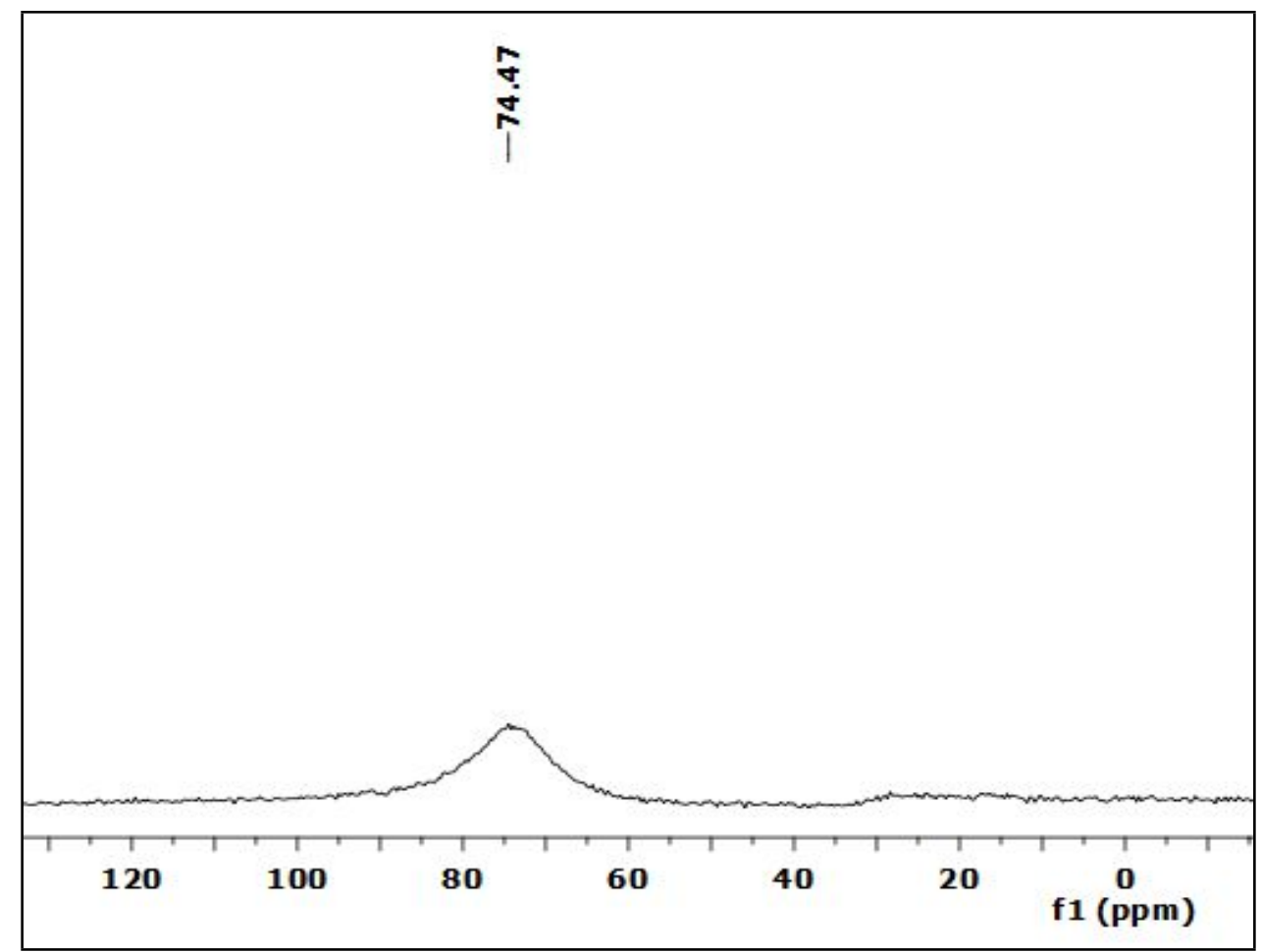

Figure S13. ${ }^{11} \mathrm{~B}$ NMR spectrum of $\mathbf{3}$

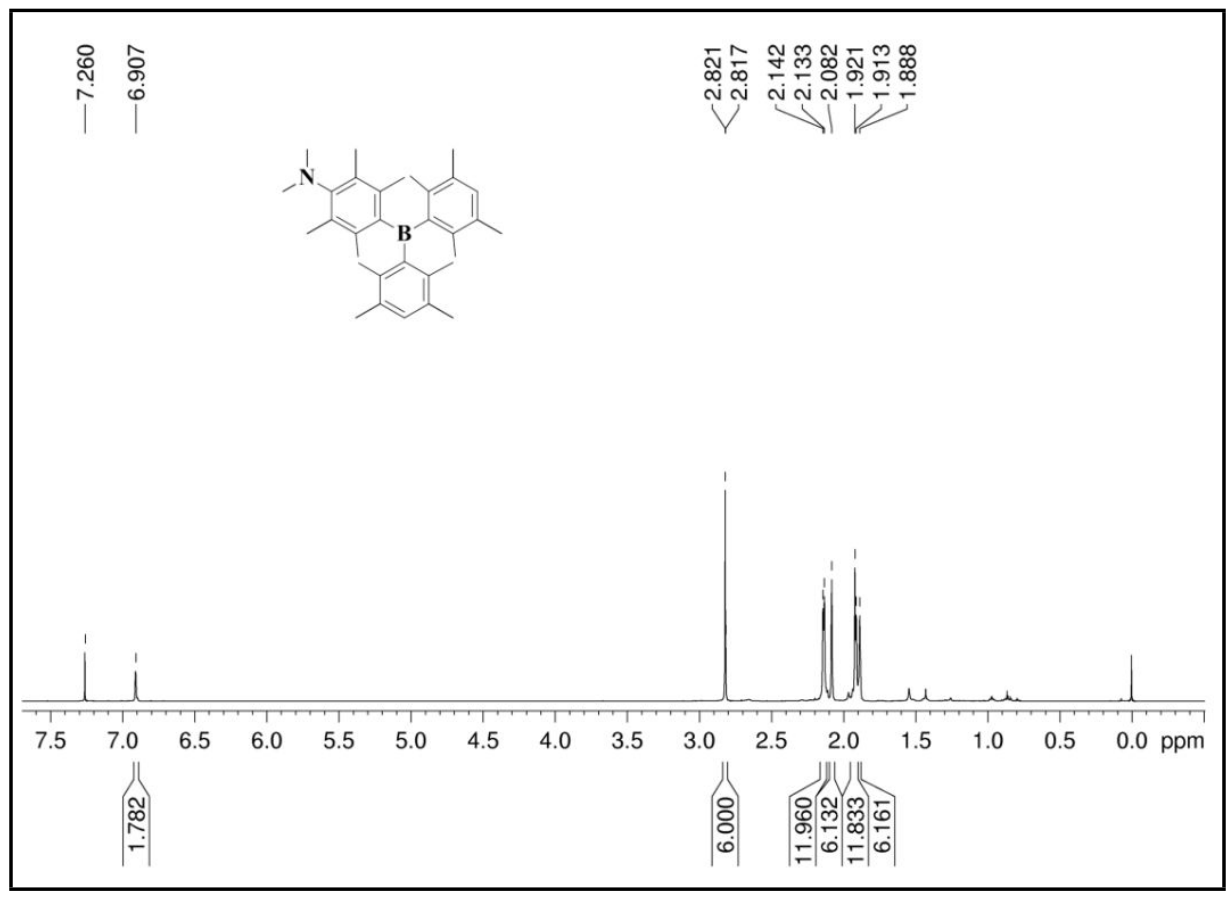

Figure S14. ${ }^{1} \mathrm{H}$ NMR spectrum of 4 


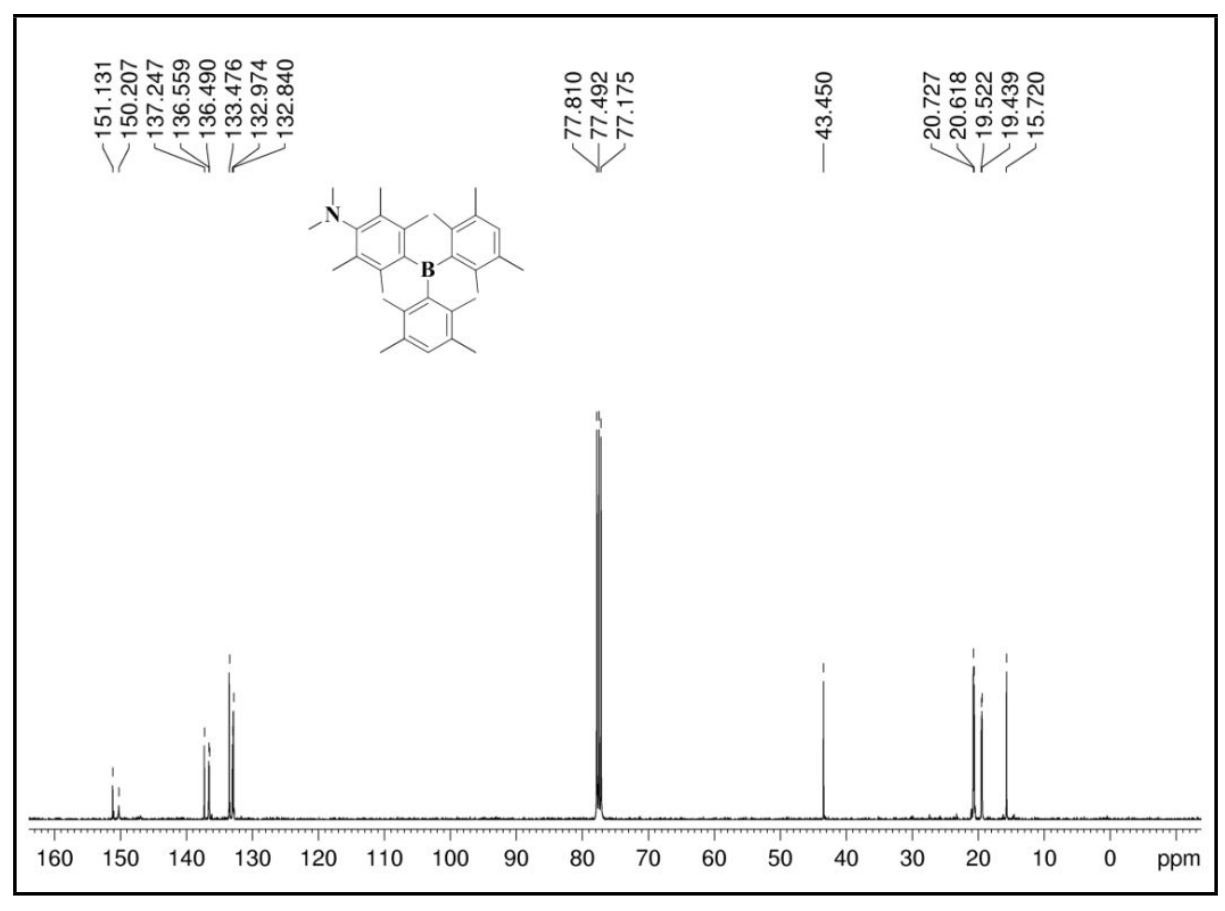

Figure S15. ${ }^{13} \mathrm{C}$ NMR spectrum of $\mathbf{4}$

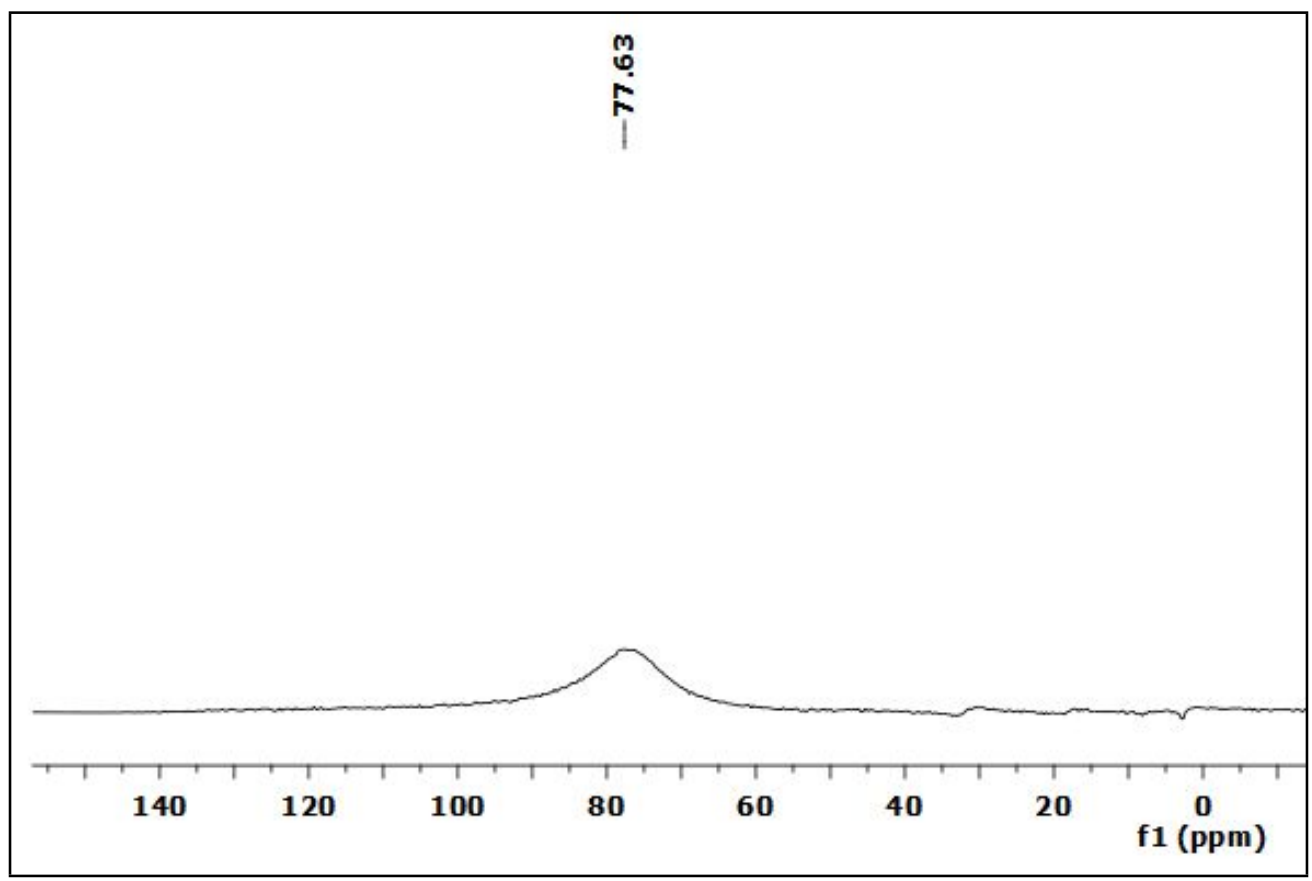

Figure S16. ${ }^{11} \mathrm{~B}$ NMR spectrum of 4 


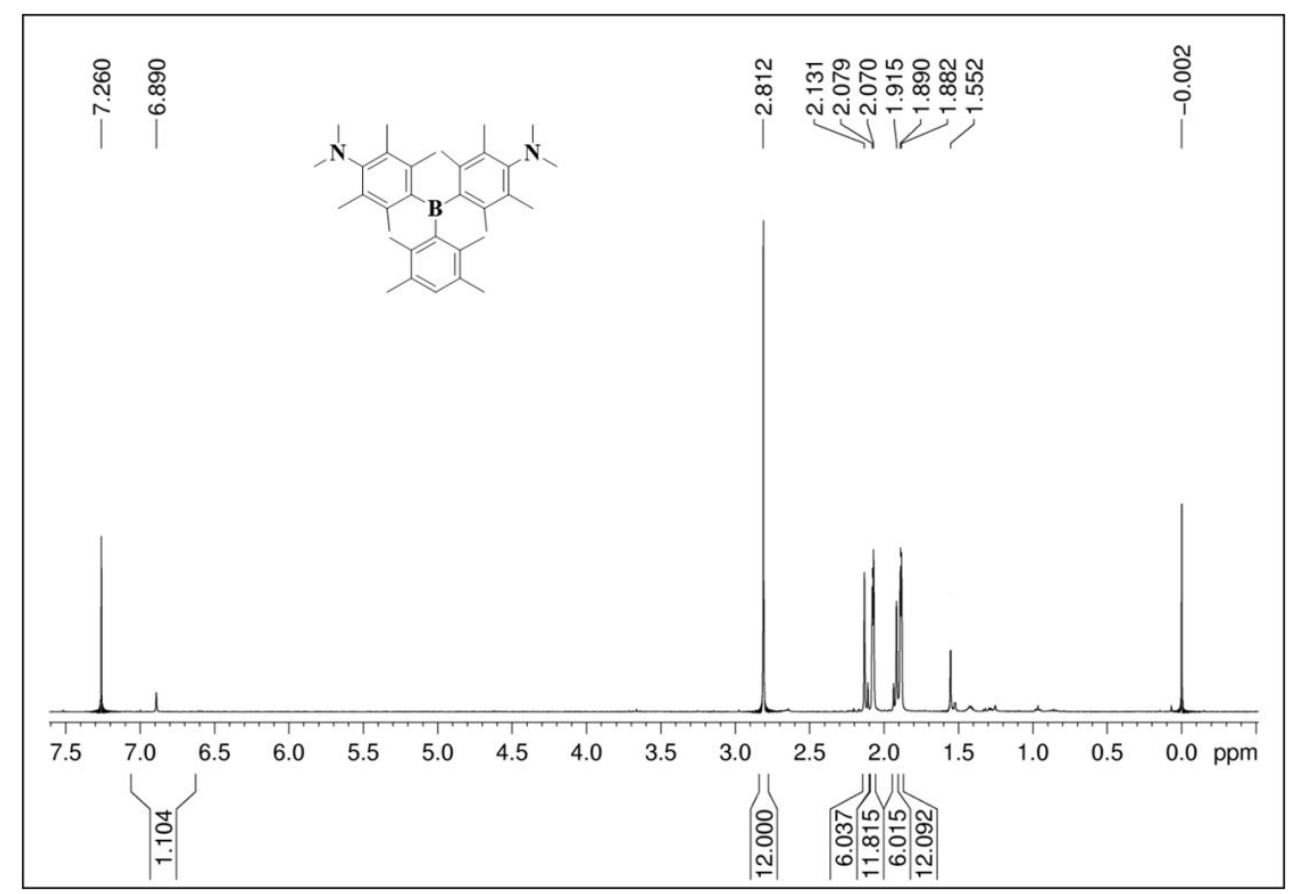

Figure S17. ${ }^{1} \mathrm{H}$ NMR spectrum of 5

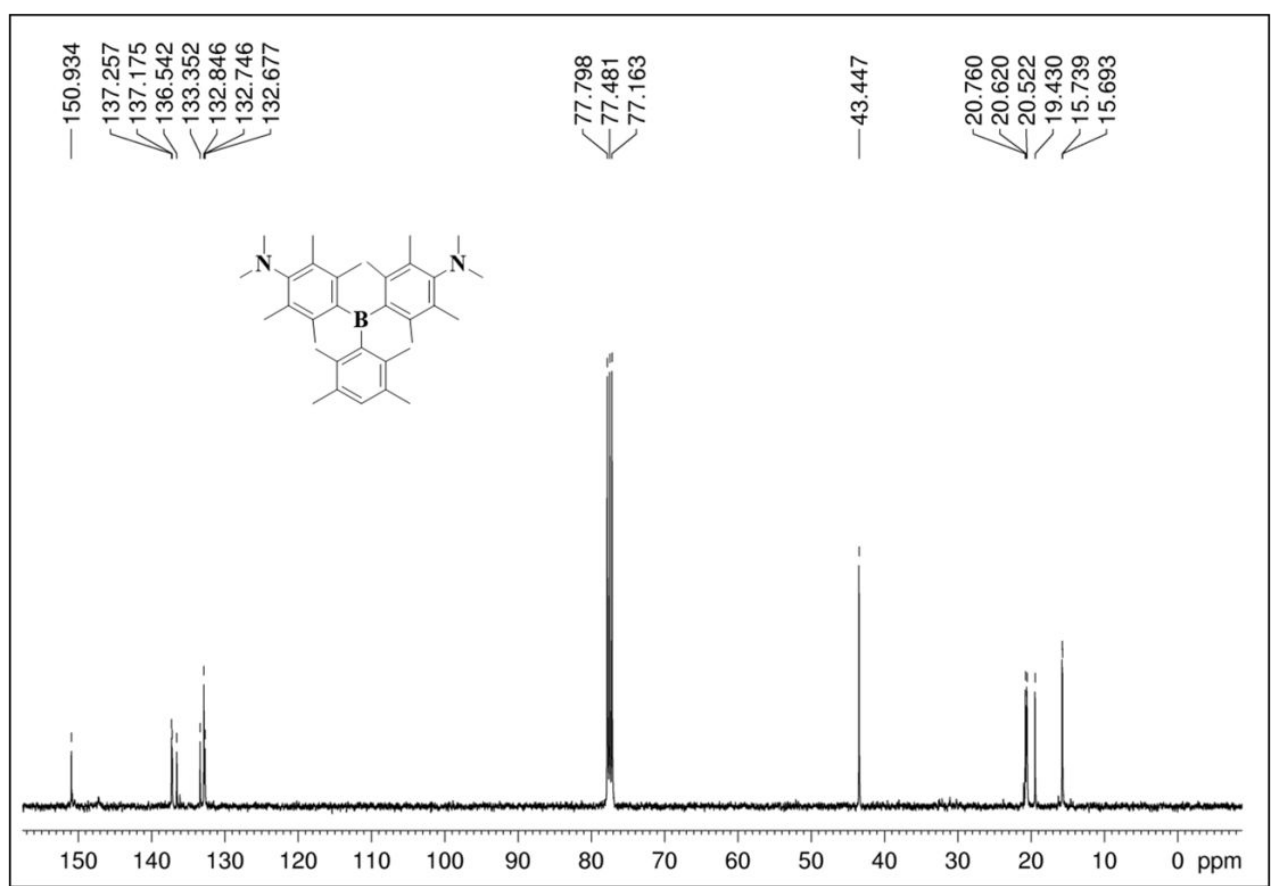

Figure S18. ${ }^{13} \mathrm{C}$ NMR spectrum of 5 


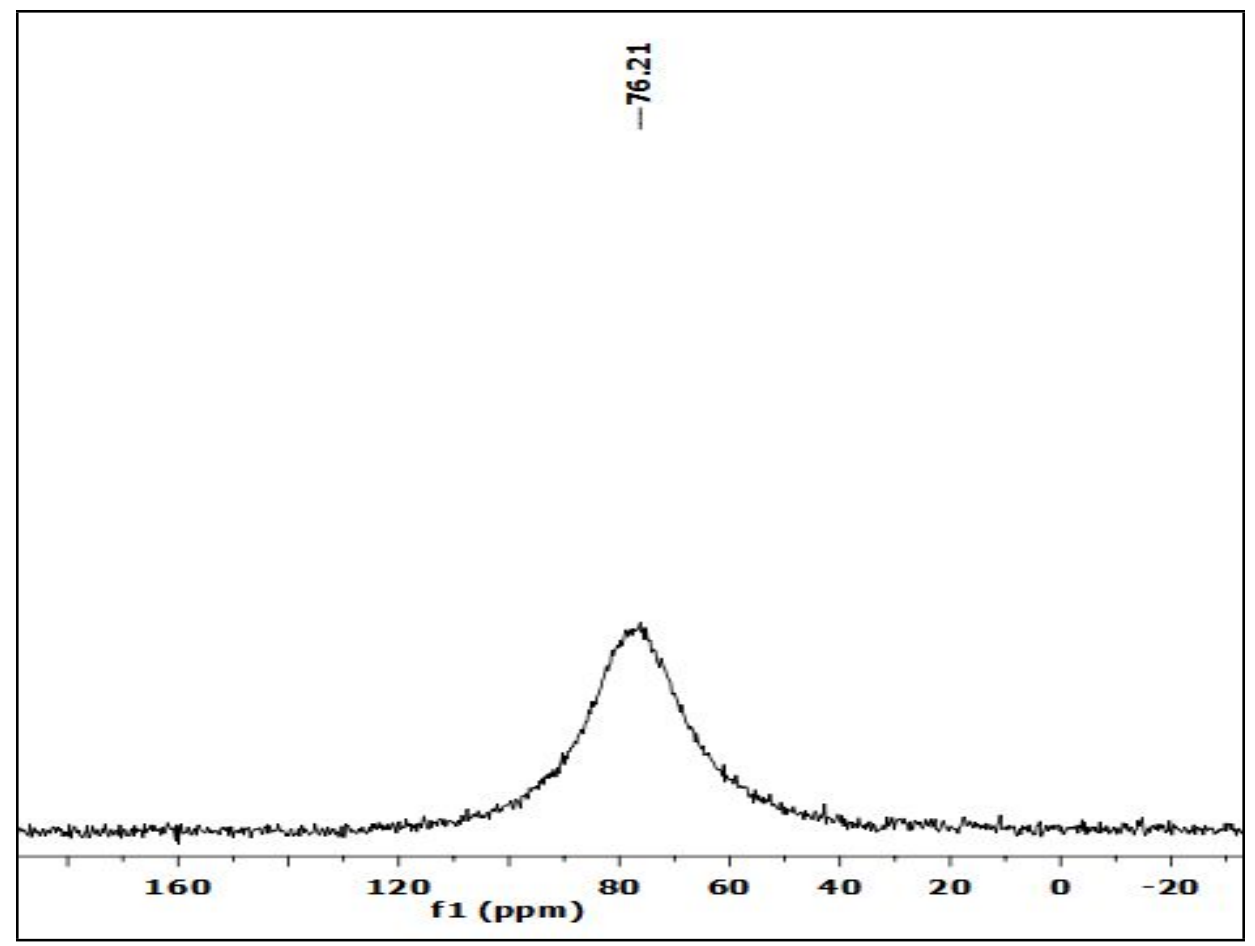

Figure S19. ${ }^{11} \mathrm{~B}$ NMR spectrum of 5

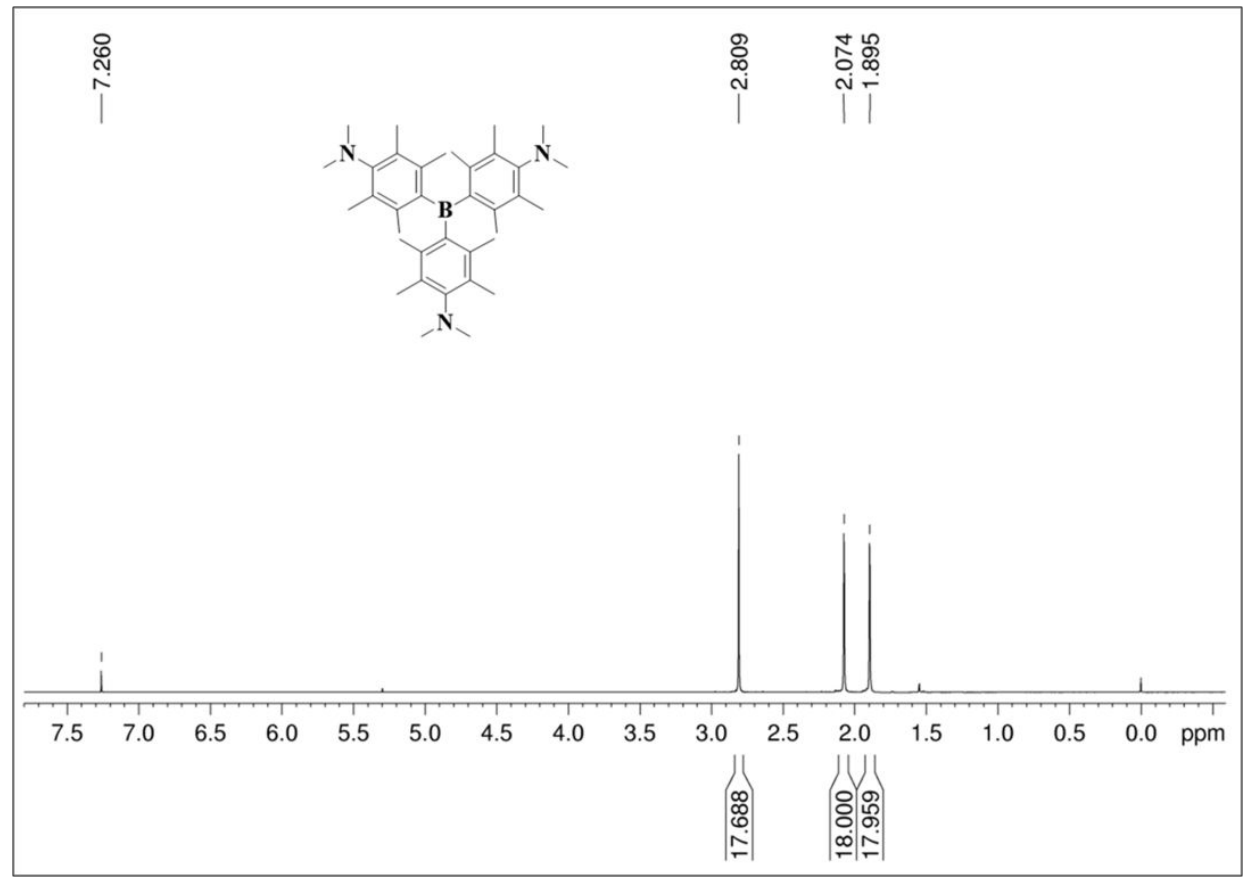

Figure S20. ${ }^{1} \mathrm{H}$ NMR spectrum of $\mathbf{6}$ 


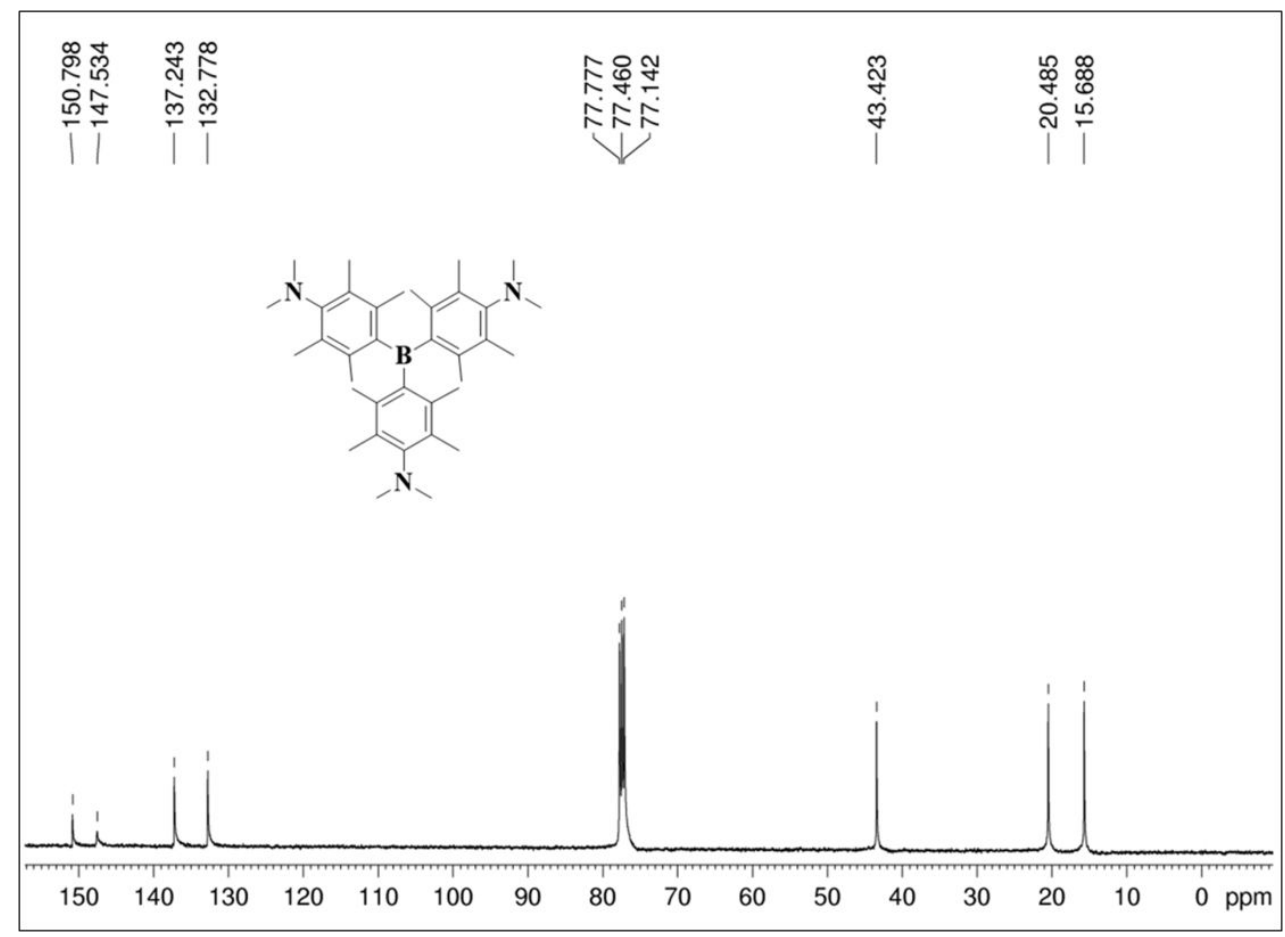

Figure S21. ${ }^{13} \mathrm{C}$ NMR spectrum of 6

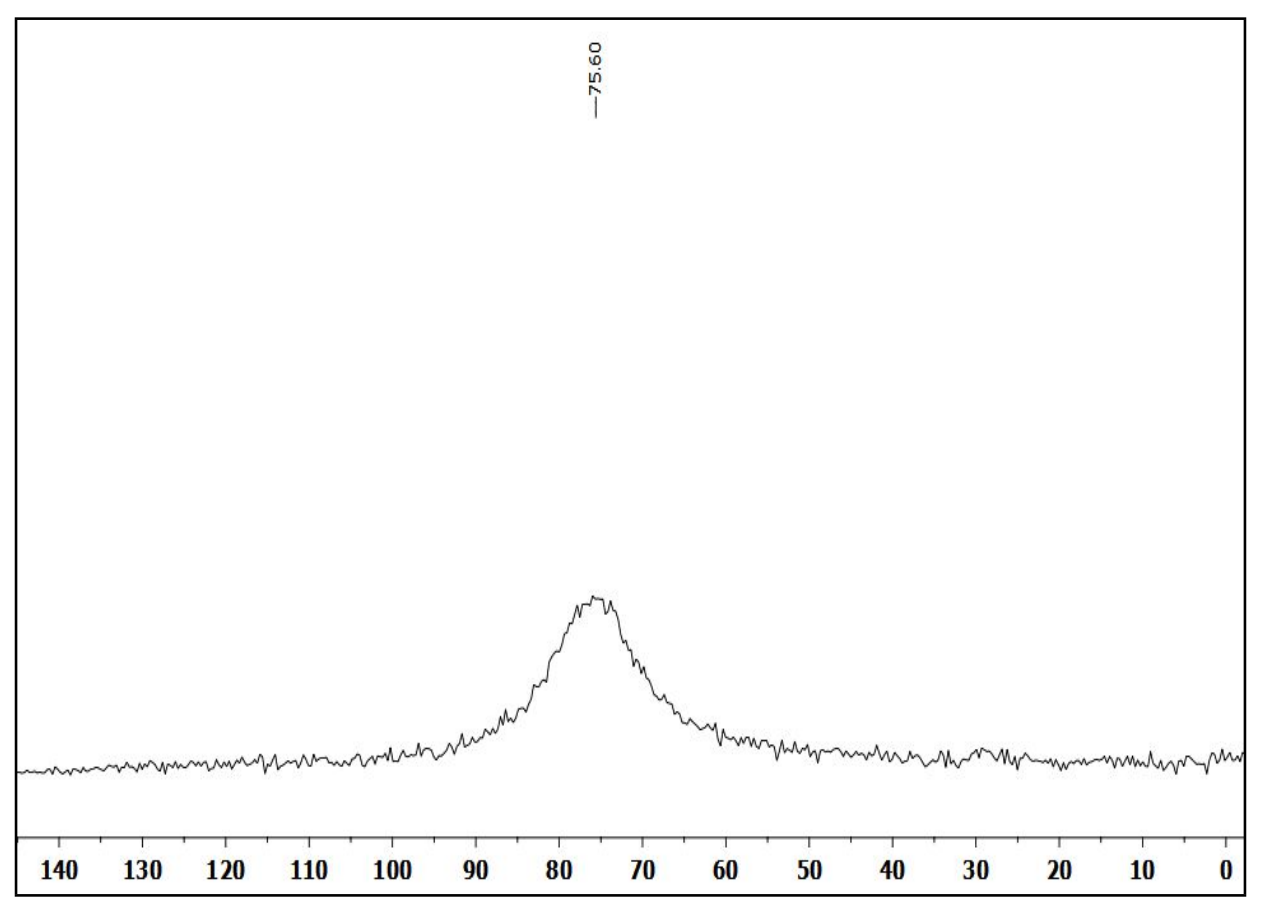

Figure S22. ${ }^{11} \mathrm{~B}$ NMR spectrum of 6 


\section{High Resolution Mass Spectral Characterization}

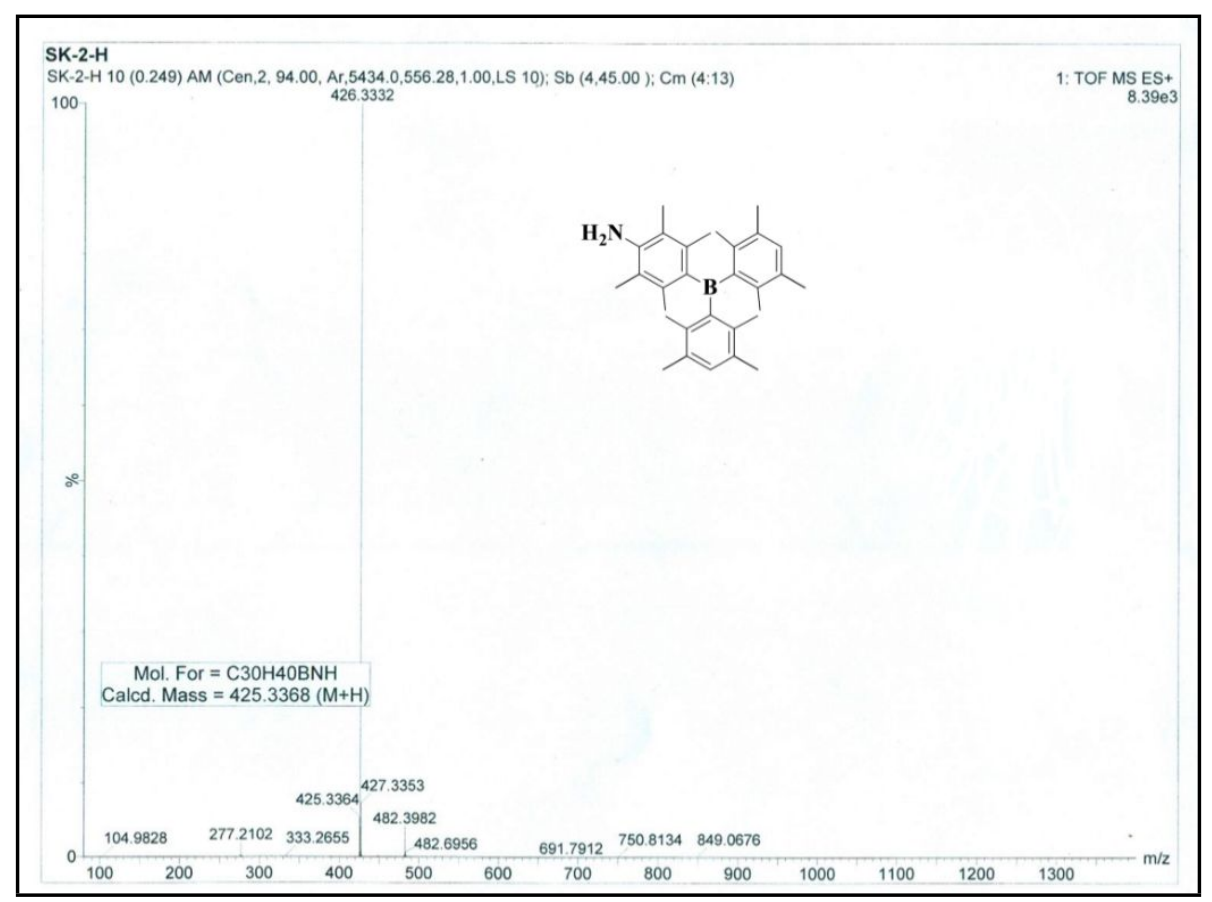

Figure S23. HRMS of 1

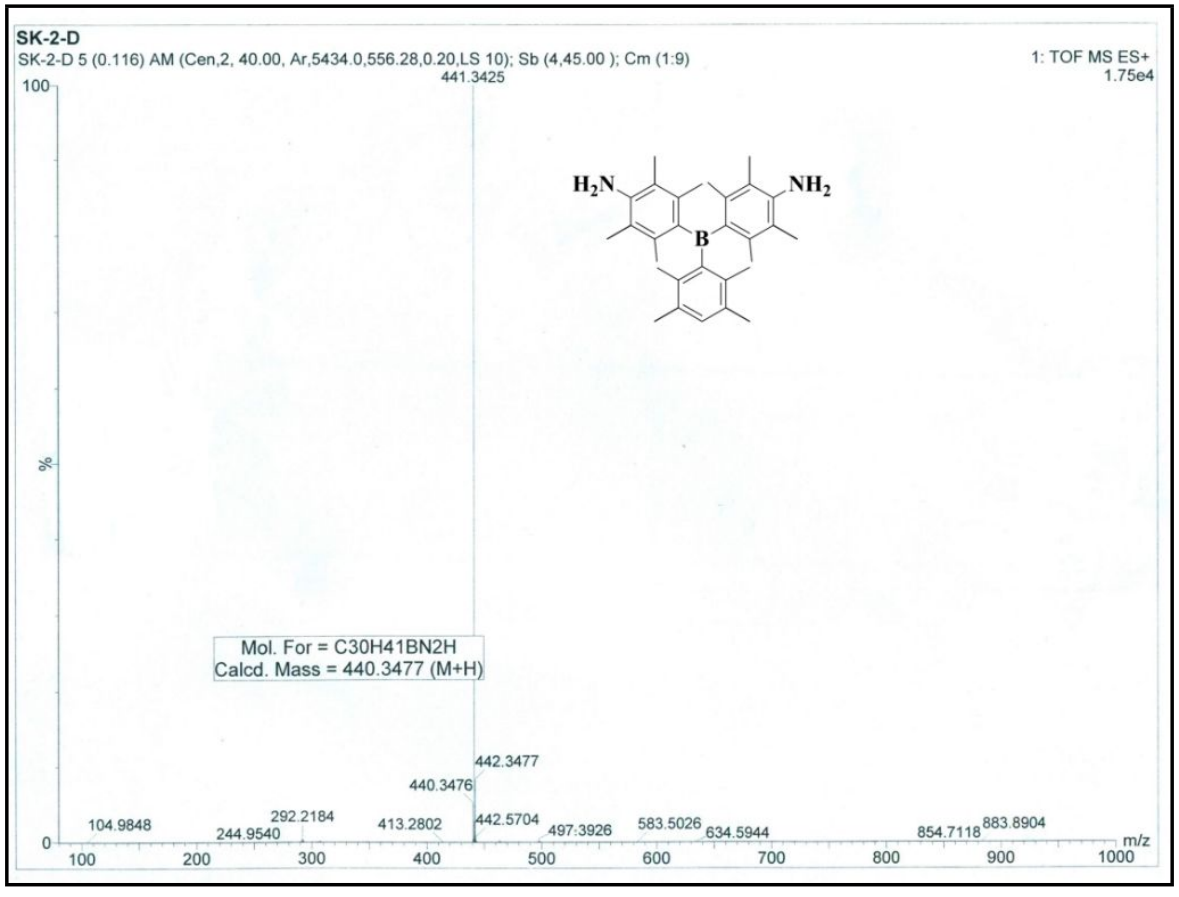

Figure S24. HRMS of 2 


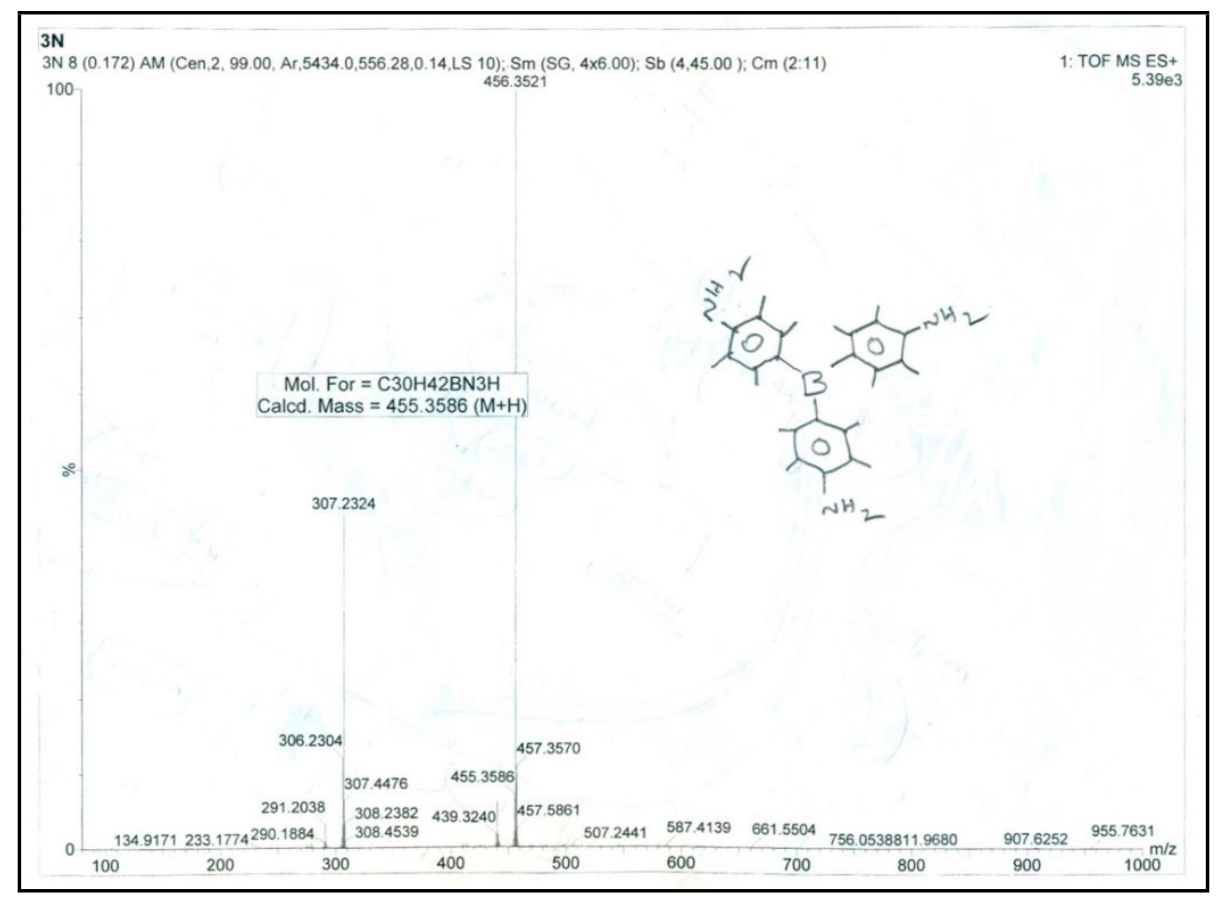

Figure S25. HRMS of $\mathbf{3}$

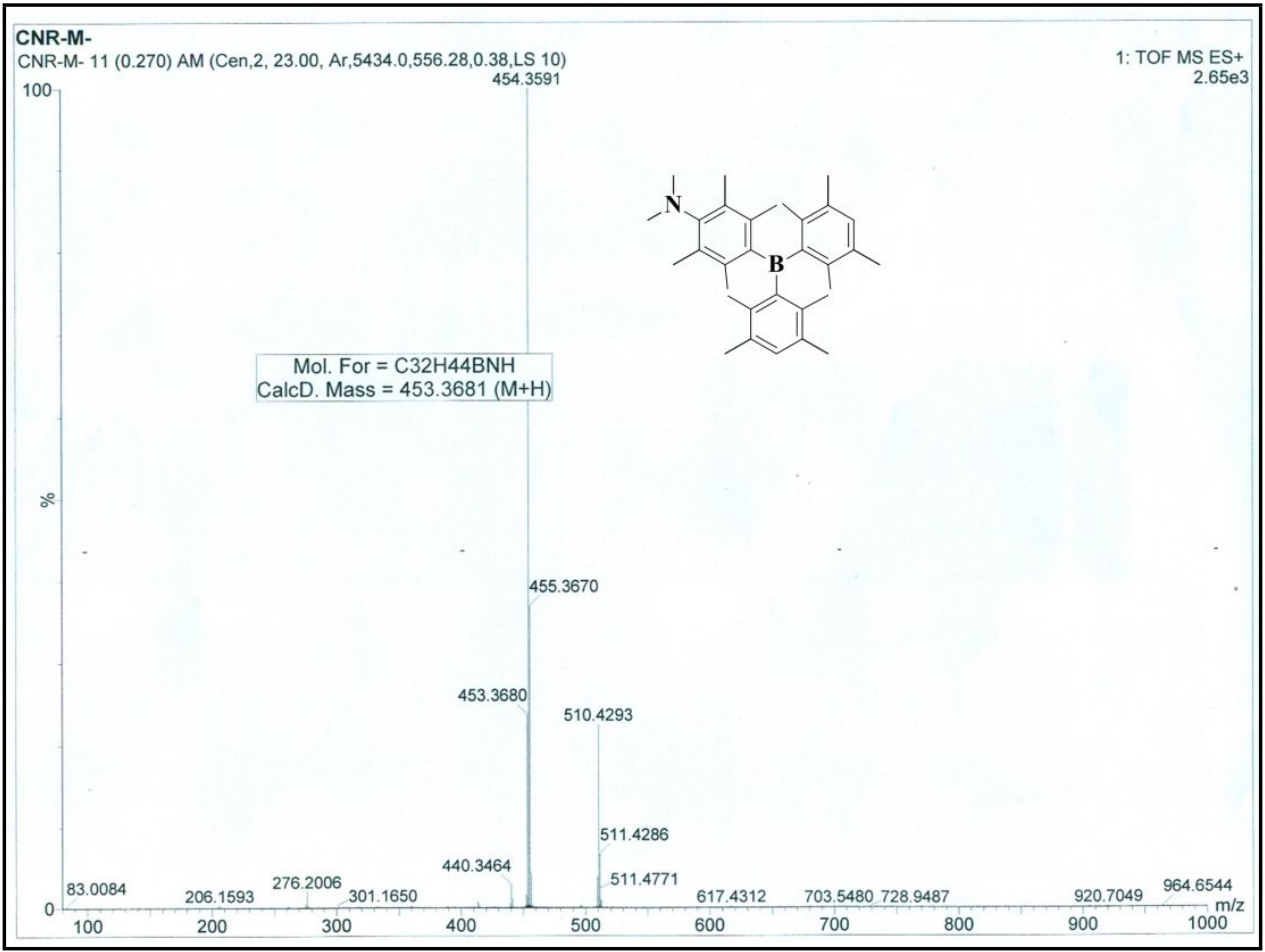

Figure S26. HRMS of 4 


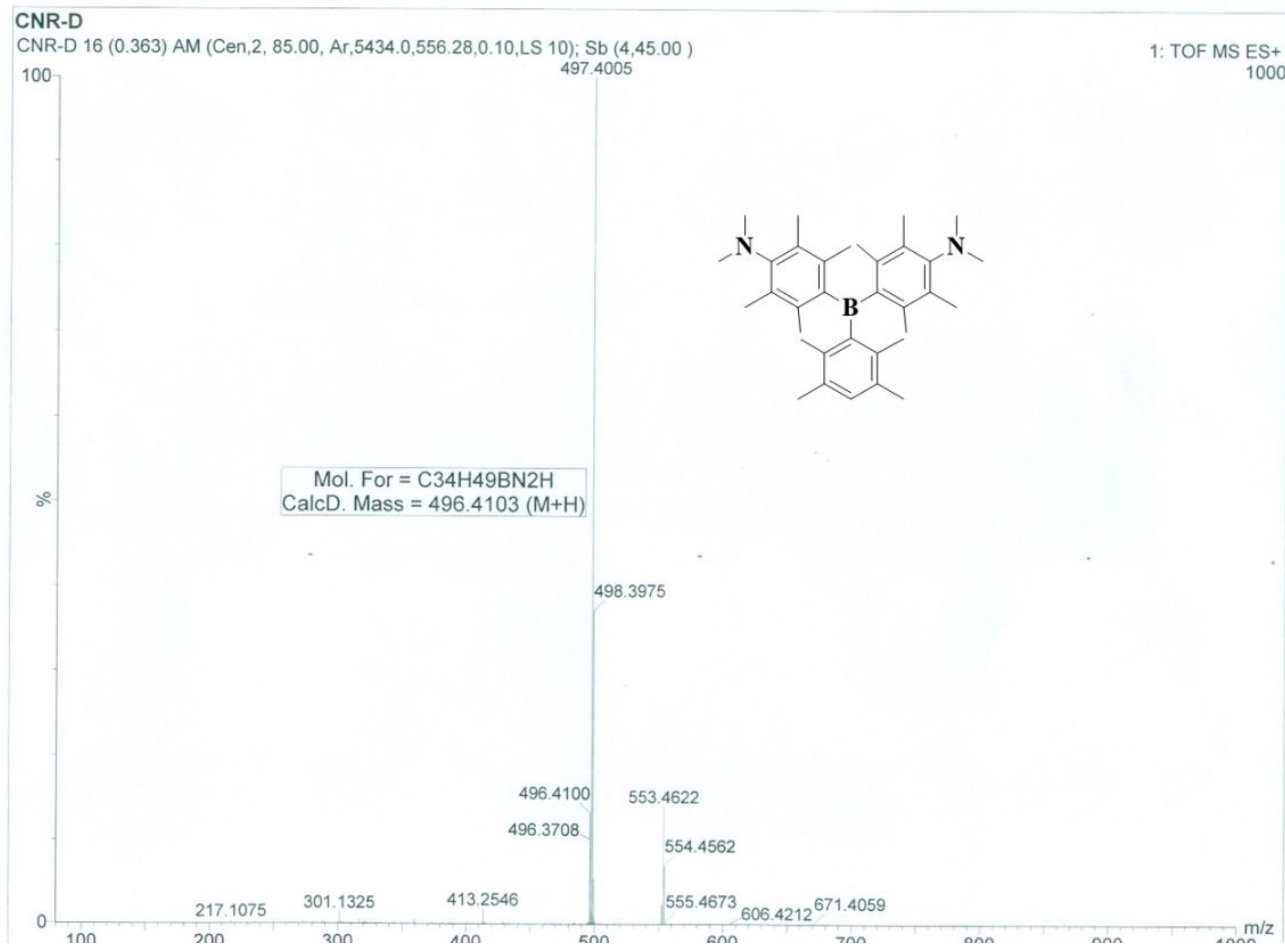

Figure S27. HRMS of 5

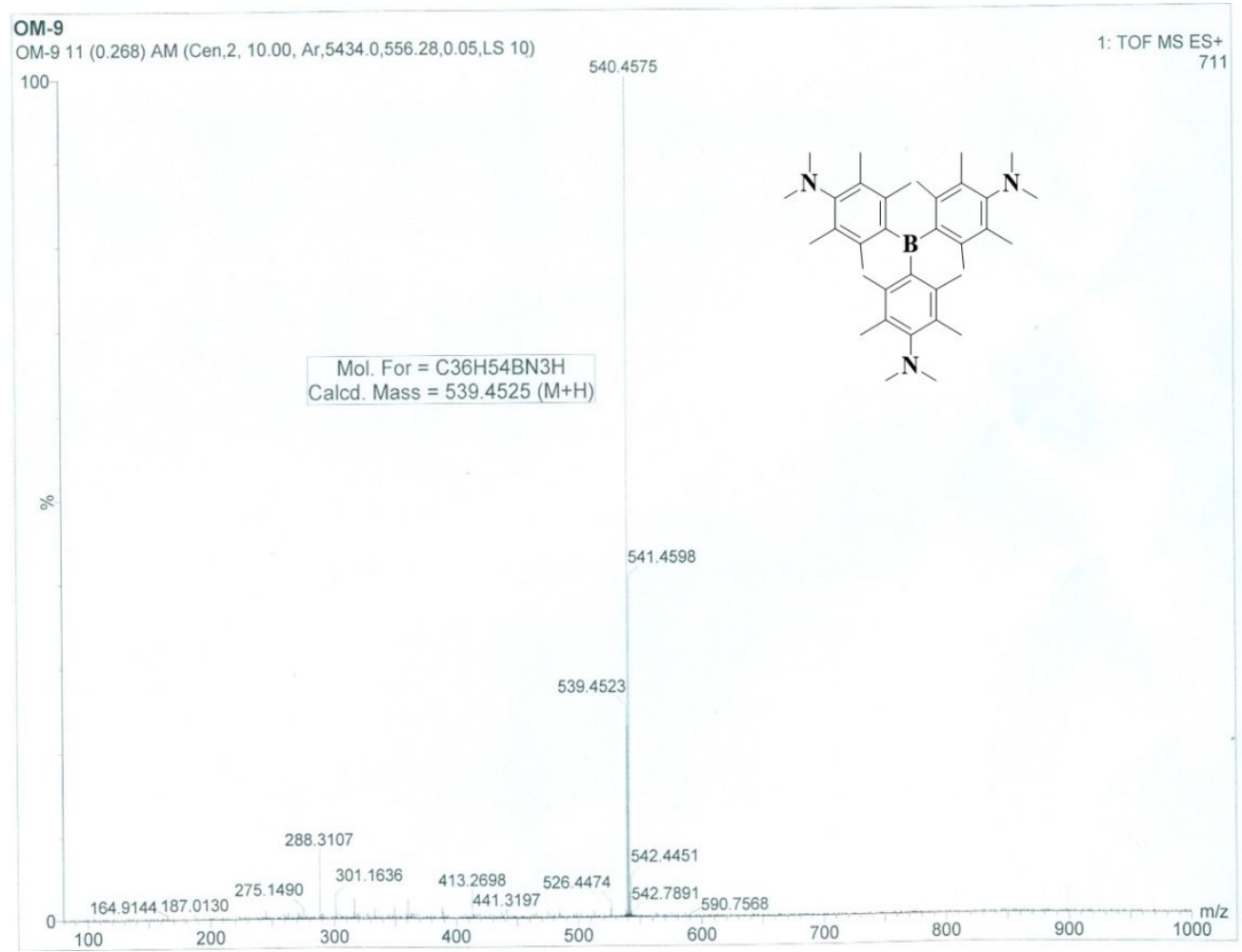

Figure S28. HRMS of $\mathbf{6}$ 


\section{Temperature Dependent ${ }^{1} \mathrm{H}$ NMR Measurements}
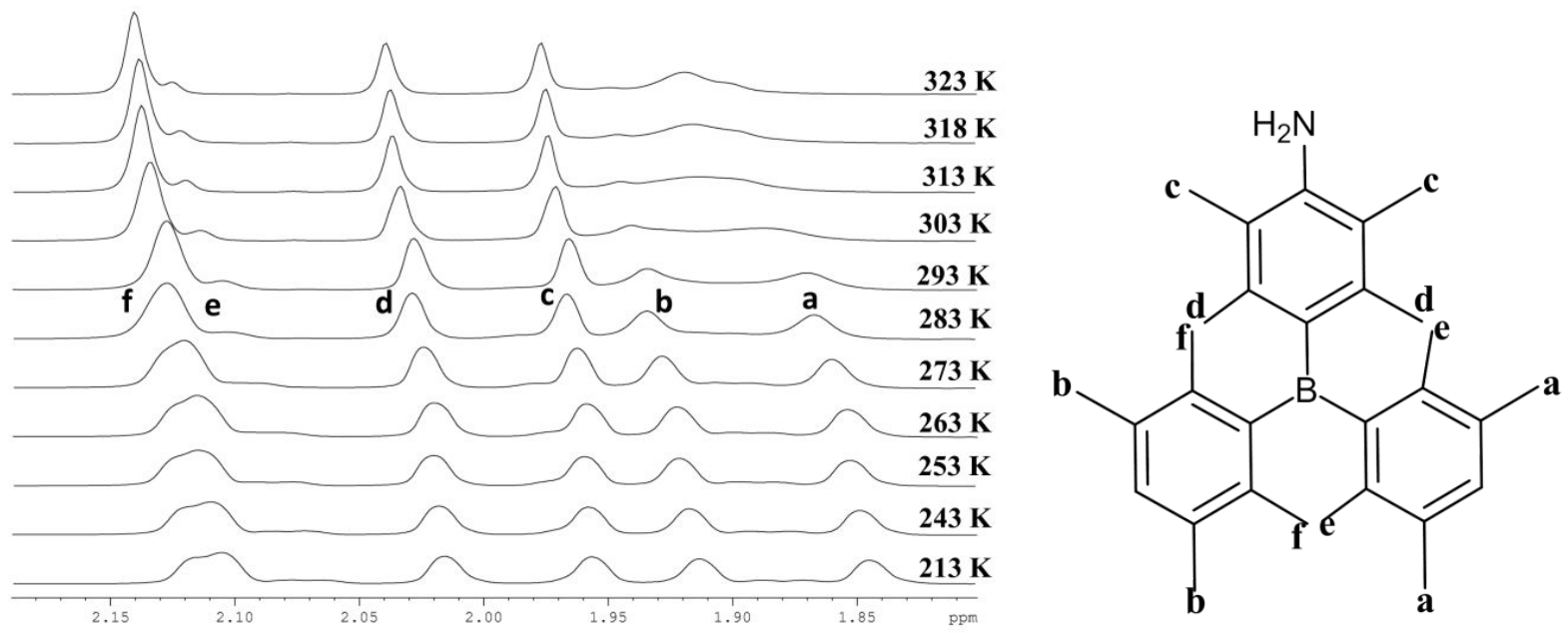

Figure S29. Stack plot of variable temperature ${ }^{1} \mathrm{H}$ NMR spectra of $\mathbf{1}$
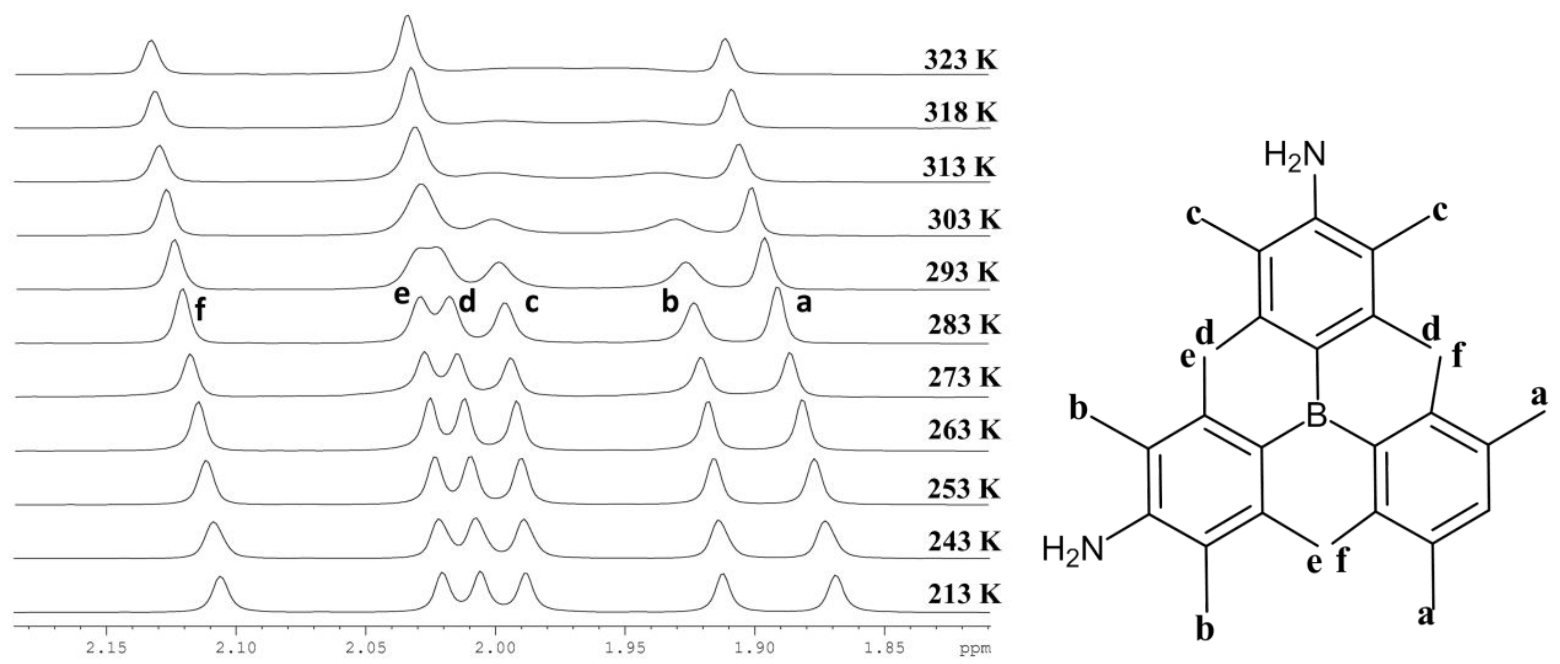

Figure S30. Stack plot of variable temperature ${ }^{1} \mathrm{H}$ NMR spectra of 2 

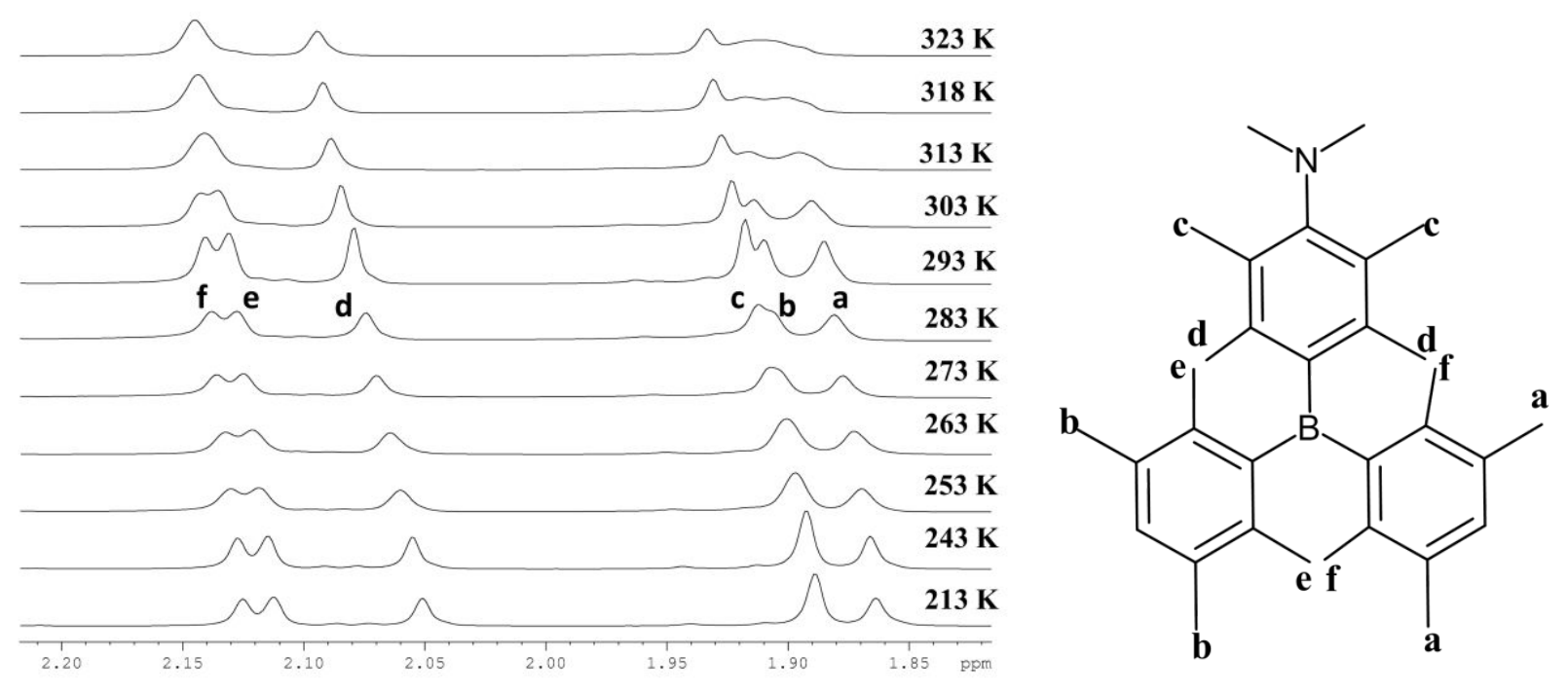

Figure S31. Stack plot of variable temperature ${ }^{1} \mathrm{H}$ NMR spectra of 4
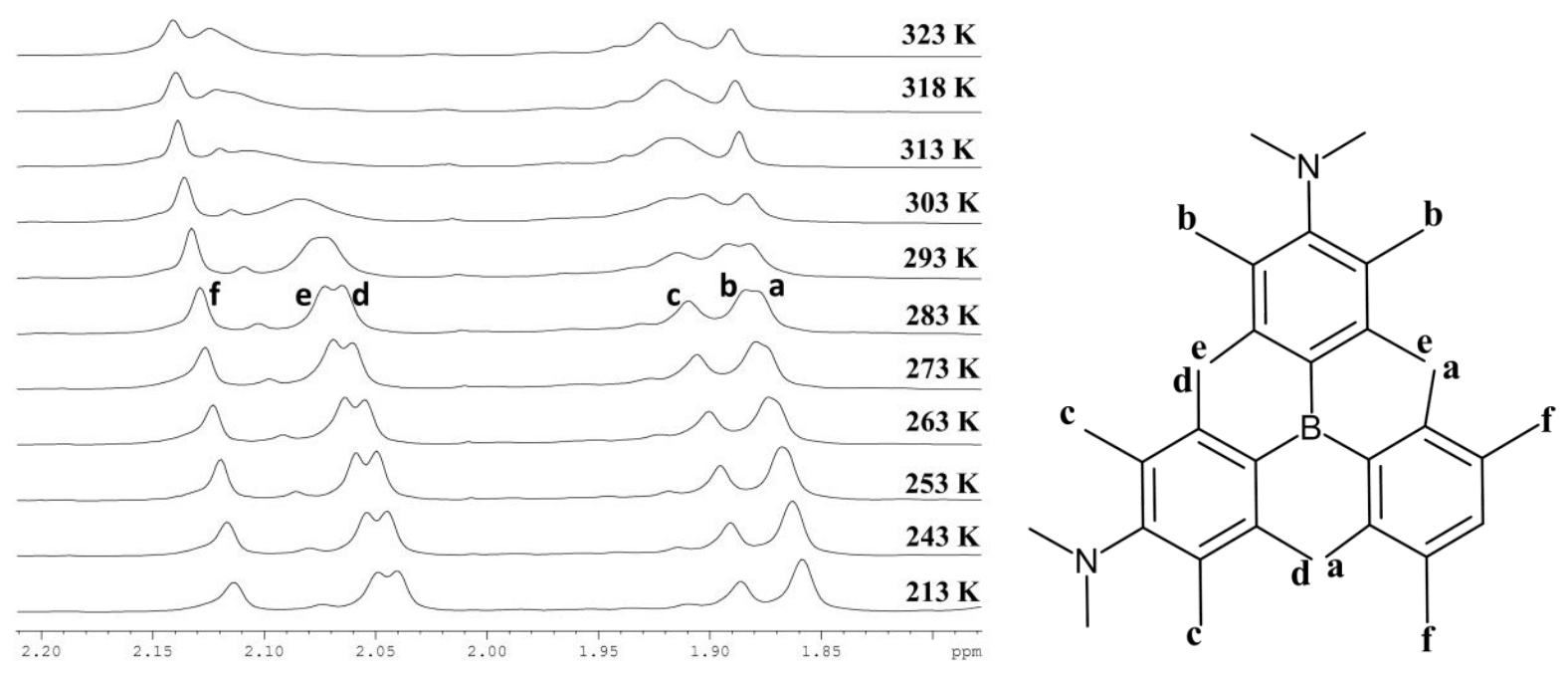

Figure S32. Stack plot of variable temperature ${ }^{1} \mathrm{H}$ NMR spectra of 5 


\section{X-ray Crystallography}
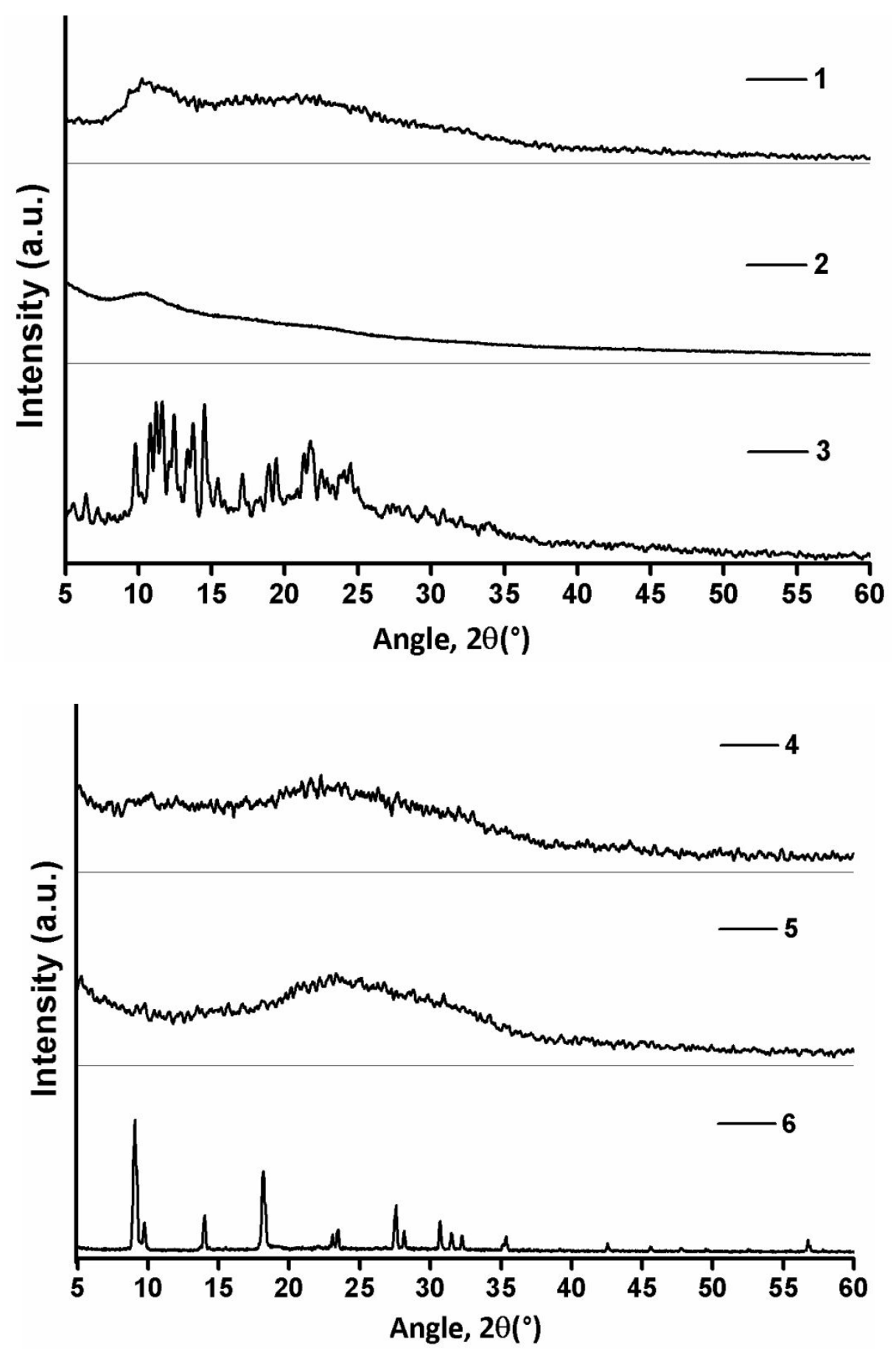

Figure S33. PXRD patterns of 1-3 (top) and 4-6 (bottom) 
Table S1. Crystallographic data of $\mathbf{3}$ and $\mathbf{6}$.

\begin{tabular}{|c|c|c|}
\hline Compound & 3 & 6 \\
\hline Formula & $\mathrm{C}_{30} \mathrm{H}_{42} \mathrm{BN}_{3}$ & $\mathrm{C}_{36} \mathrm{H}_{54} \mathrm{BN}_{3}$ \\
\hline Formula weight & 455.48 & 539.44 \\
\hline System & Triclinic & Monoclinic \\
\hline Space group & $P-1$ & $C 2 / c$ \\
\hline $\mathrm{a}(\AA)$ & $9.398(2)$ & $12.900(1)$ \\
\hline $\mathrm{b}(\AA)$ & $12.856(3)$ & $12.847(1)$ \\
\hline $\mathrm{c}(\AA)$ & $13.331(3)$ & $19.988(2)$ \\
\hline$\alpha /^{\circ}$ & $96.42(2)$ & 90.00 \\
\hline$\beta /^{\circ}$ & $93.84(1)$ & $105.51(6)$ \\
\hline$\gamma /{ }^{\circ}$ & $111.41(1)$ & 90.00 \\
\hline $\mathrm{V} / \AA^{3}$ & $1479.9(5)$ & $3192.2(5)$ \\
\hline Crystal size $(\mathrm{mm})$ & $0.10 \times 0.09 \times 0.07$ & $0.10 \times 0.09 \times 0.080$ \\
\hline $\mathrm{Z}$ & 2 & 8 \\
\hline Density $(\mathrm{gcm}-3)$ & 1.184 & 1.119 \\
\hline Final R $[I>2 s(I)]$ & $\begin{array}{c}\mathrm{R} 1=0.0701, \mathrm{wR} 2= \\
0.1783\end{array}$ & $\begin{array}{c}\mathrm{R} 1=0.0448, \mathrm{wR} 2= \\
0.1351\end{array}$ \\
\hline R (all data) & $\begin{array}{c}\mathrm{R} 1=0.1413, \mathrm{wR} 2= \\
0.2049\end{array}$ & $\begin{array}{c}\mathrm{R} 1=0.0520, \mathrm{wR} 2= \\
0.1296\end{array}$ \\
\hline Collected reflns & 18437 & 26670 \\
\hline Unique reflns & 5073 & 3146 \\
\hline Theta range for data collection $\left({ }^{\circ}\right)$ & 1.5 to 25 & 2.1 to 26.0 \\
\hline Absorption coefficient $\left(\mathrm{mm}^{-1}\right)$ & 0.070 & 0.066 \\
\hline GOOF & 1.009 & 1.045 \\
\hline CCDC No. & 993251 & 993252 \\
\hline
\end{tabular}

${ }^{[\mathrm{a}]} R_{1}=\Sigma|| F_{\mathrm{o}}|-| F_{\mathrm{c}}|| / \Sigma\left|F_{\mathrm{o}}\right|,{ }^{[\mathrm{b}]}{ }_{w} R_{2}=\left[\Sigma\left\{w\left(F_{\mathrm{o}}{ }^{2}-F_{\mathrm{c}}{ }^{2}\right)^{2}\right\} / \Sigma\left\{w\left(F_{\mathrm{o}}{ }^{2}\right)^{2}\right\}\right]^{1 / 2}$ 


\section{Spectroscopic Characterization}
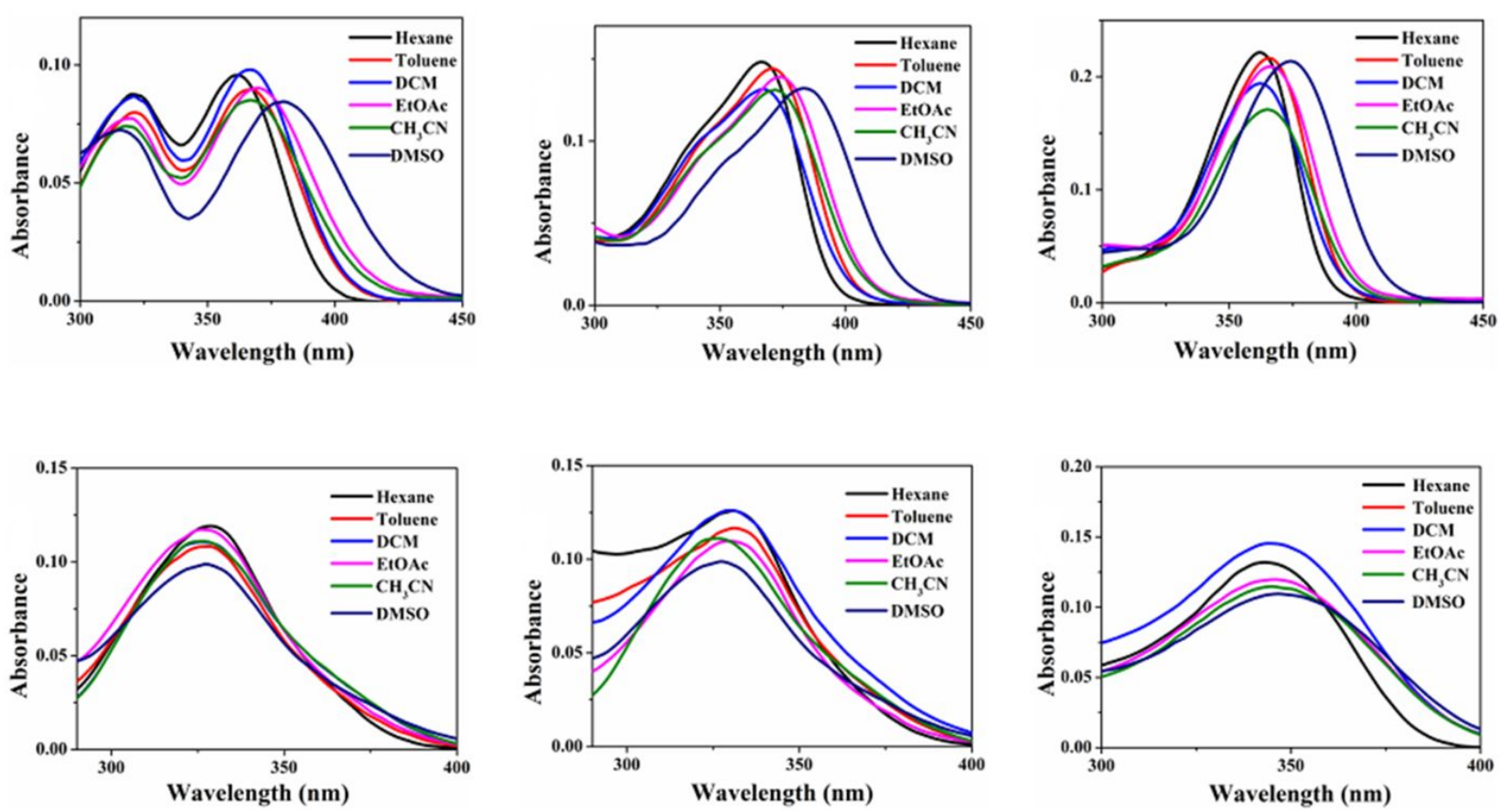

Figure S34. Absorption spectra of 1-3 (top left to right) and 4-6 (bottom left to right) in solvents of different polarity $($ Concentration $=10 \mu \mathrm{M})$ 

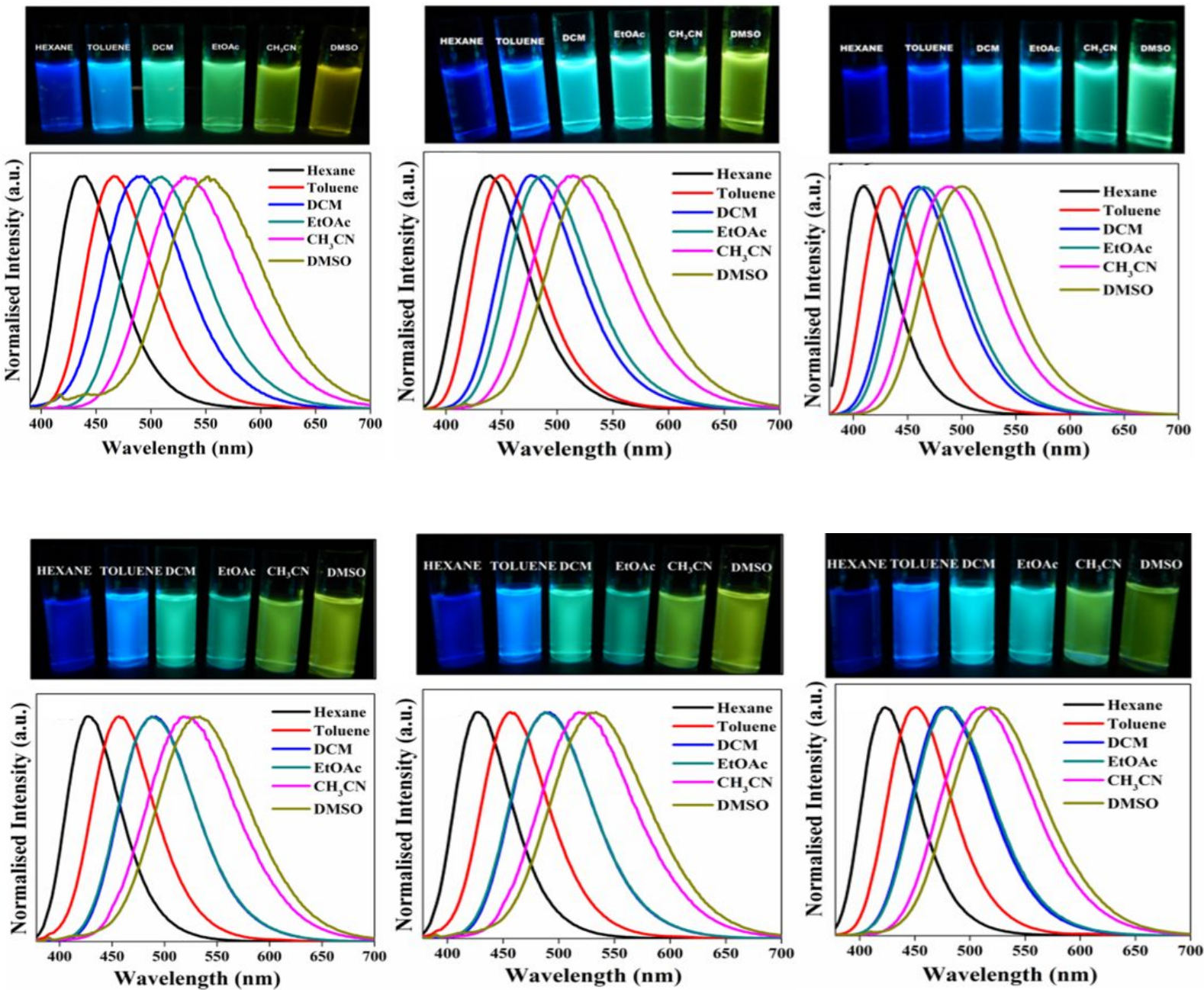

Figure S35. Fluorescence emission spectra of 1-3 (top left to right) and 4-6 (bottom left to right) in solvents of different polarity (Concentration $=10 \mu \mathrm{M}, \lambda_{\mathrm{ex}}=370 \mathrm{~nm}$ for 1-3 and $330 \mathrm{~nm}$ for 4-6). Top inset shows the images of compounds in different solvents under UV light irradation (365 nm). 
Table S2. Photophysical properties of 1-6 in solvents of different polarity (Concentration $=10 \mu \mathrm{M}, \lambda_{\mathrm{ex}}=$ $370 \mathrm{~nm}$ for 1-3 and $330 \mathrm{~nm}$ for 4-6, quantum yield $\Phi_{\mathrm{F}}$ is calculated with respect to anthracene $\mathrm{e}^{\mathrm{ref}}$ ).

\begin{tabular}{|c|c|c|c|c|c|}
\hline Compound No. & Solvent & $\lambda_{\text {abs }}(\mathrm{nm})(\varepsilon)\left(\mathbf{M}^{-1} \mathbf{c m}^{-1}\right)$ & $\lambda_{\mathrm{em}}(\mathbf{n m})$ & Stokes shift $\left(\mathrm{cm}^{-1}\right)$ & $\boldsymbol{\Phi}_{\mathrm{F}}$ \\
\hline \multirow{6}{*}{1} & Hexane & $362(9559), 321$ (8763) & 437 & 4741 & 0.30 \\
\hline & Toluene & $367(8998), 322$ (7965) & 467 & 5834 & 0.52 \\
\hline & $\mathrm{DCM}$ & $362(8604), 321(8147)$ & 490 & 7216 & 0.46 \\
\hline & EtOAc & $371(8984), 320(7728)$ & 508 & 7269 & 0.30 \\
\hline & $\mathrm{CH}_{3} \mathrm{CN}$ & $367(8486), 319(7381)$ & 531 & 8415 & 0.18 \\
\hline & DMSO & $380(8431), 317(7217)$ & 554 & 8265 & 0.12 \\
\hline \multirow{6}{*}{2} & Hexane & $366(14866)$ & 439 & 4543 & 0.20 \\
\hline & Toluene & $371(14427)$ & 450 & 4731 & 0.55 \\
\hline & $\mathrm{DCM}$ & $366(13198)$ & 476 & 6314 & 0.43 \\
\hline & EtOAc & $373(13895)$ & 487 & 6275 & 0.32 \\
\hline & $\mathrm{CH}_{3} \mathrm{CN}$ & $372(13175)$ & 513 & 7388 & 0.22 \\
\hline & DMSO & $383(13304)$ & 529 & 7206 & 0.14 \\
\hline \multirow{6}{*}{3} & Hexane & $362(22157)$ & 409 & 3174 & 0.11 \\
\hline & Toluene & $365(21662)$ & 432 & 4219 & 0.18 \\
\hline & DCM & $362(19433)$ & 460 & 5885 & 0.28 \\
\hline & EtOAc & $366(20931)$ & 465 & 5817 & 0.26 \\
\hline & $\mathrm{CH}_{3} \mathrm{CN}$ & $365(17073)$ & 488 & 6905 & 0.30 \\
\hline & DMSO & $374(21373)$ & 500 & 6737 & 0.46 \\
\hline \multirow{6}{*}{4} & Hexane & $328(11957)$ & 441 & 7812 & 0.15 \\
\hline & Toluene & $327(10825)$ & 457 & 8699 & 0.28 \\
\hline & DCM & $326(11110)$ & 488 & 10183 & 0.36 \\
\hline & EtOAc & 327 (11764) & 488 & 10089 & 0.20 \\
\hline & $\mathrm{CH}_{3} \mathrm{CN}$ & $326(11093)$ & 519 & 11407 & 0.13 \\
\hline & DMSO & $326(9881)$ & 530 & 11806 & 0.20 \\
\hline \multirow{6}{*}{5} & Hexane & $330(12721)$ & 428 & 6938 & 0.11 \\
\hline & Toluene & 331 (11628) & 456 & 8281 & 0.23 \\
\hline & DCM & $330(12787)$ & 485 & 9684 & 0.32 \\
\hline & EtOAc & $330(10943)$ & 487 & 9769 & 0.20 \\
\hline & $\mathrm{CH}_{3} \mathrm{CN}$ & $331(10706)$ & 518 & 10906 & 0.14 \\
\hline & DMSO & $331(12616)$ & 525 & 11163 & 0.20 \\
\hline \multirow{6}{*}{6} & Hexane & $343(13222)$ & 423 & 5513 & 0.19 \\
\hline & Toluene & $344(12508)$ & 451 & 6896 & 0.25 \\
\hline & DCM & $345(14562)$ & 477 & 8021 & 0.38 \\
\hline & EtOAc & $344(12508)$ & 478 & 8149 & 0.28 \\
\hline & $\mathrm{CH}_{3} \mathrm{CN}$ & 344 (11378) & 511 & 9500 & 0.24 \\
\hline & DMSO & 348 (10939) & 520 & 9504 & 0.41 \\
\hline
\end{tabular}


Table S3. Ground state and excited state dipole moments of 1-6

\begin{tabular}{|c|c|c|c|c|c|c|}
\hline & $\mathbf{1}$ & $\mathbf{2}$ & $\mathbf{3}$ & $\mathbf{4}$ & $\mathbf{5}$ & $\mathbf{6}$ \\
\hline$\mu_{g}$ & 2.13 & 0.65 & 1.55 & 0.51 & 0.17 & 0.60 \\
\hline$\mu_{e}$ & 13.23 & 13.13 & 13.94 & 15.0 & 15.2 & 15.7 \\
\hline$\Delta \mu_{e g}$ & 11.1 & 12.48 & 12.39 & 14.49 & 15.03 & 15.1 \\
\hline
\end{tabular}

$\left(\mu_{g}=\right.$ ground state dipole moment obtained from DFT calculations, $\mu_{e}=$ excited state dipole moment and $\Delta \mu_{e g}=$ change in dipole moment estimated from Lippert-Mataga plots).

Table S4. Photophysical properties of compounds 1-6 in solvents of different polarity $\left(\lambda_{\mathrm{ex}}=370 \mathrm{~nm}\right.$ for 1-3 and $330 \mathrm{~nm}$ for 4-6)

\begin{tabular}{|c|c|c|c|c|c|}
\hline & & $\lambda_{\mathrm{em}}(\mathrm{nm})$ & $\tau / \mathrm{ns}$ & $\chi^{2}$ & $\Phi_{\mathrm{F}}$ \\
\hline \multirow[t]{3}{*}{1} & Hexane & 437 & $\begin{array}{l}\tau_{1}=4.86(25 \%) \\
\tau_{2}=6.74(75 \%)\end{array}$ & 1.2 & 0.30 \\
\hline & DCM & 490 & $\tau=12.45(100 \%)$ & 1.2 & 0.46 \\
\hline & DMSO & 554 & $\tau=12.10(100 \%)$ & 1.0 & 0.12 \\
\hline \multirow[t]{3}{*}{2} & Hexane & 439 & $\begin{array}{l}\tau_{1}=3.26(97 \%) \\
\tau_{2}=8.50(3 \%)\end{array}$ & 1.1 & 0.20 \\
\hline & DCM & 476 & $\tau=8.31(100 \%)$ & 1.1 & 0.14 \\
\hline & DMSO & 529 & $\tau=8.50(100 \%)$ & 1.2 & \\
\hline \multirow[t]{3}{*}{3} & Hexane & 409 & $\tau=1.69(100 \%)$ & 1.00 & 0.11 \\
\hline & DCM & 460 & $\tau=6.05(100 \%)$ & 1.2 & 0.28 \\
\hline & DMSO & 500 & $\tau=12.28(100 \%)$ & 1.2 & 0.46 \\
\hline \multirow[t]{3}{*}{4} & Hexane & 441 & $\begin{array}{l}\tau_{1}=3.45(66 \%) \\
\tau_{2}=6.52(34 \%)\end{array}$ & 1.1 & 0.15 \\
\hline & DCM & 488 & $\begin{array}{l}\tau_{1}=10.89(94 \%) \\
\tau_{2}=18.63(6 \%)\end{array}$ & 1.1 & 0.36 \\
\hline & DMSO & 530 & $\begin{array}{l}\tau_{1}=8.74(17 \%) \\
\tau_{2}=19.03(83 \%)\end{array}$ & 1.2 & 0.20 \\
\hline \multirow[t]{3}{*}{5} & Hexane & 423 & $\begin{array}{l}\tau_{1}=2.89(96) \% \\
\tau_{2}=8.49(4 \%)\end{array}$ & 1.1 & 0.11 \\
\hline & DCM & 485 & $\tau=10.83(100 \%)$ & 1.2 & 0.32 \\
\hline & DMSO & 525 & $\tau=17.46(100 \%)$ & 1.1 & 0.20 \\
\hline \multirow[t]{3}{*}{6} & Hexane & 423 & $\tau=2.38(100 \%)$ & 1.0 & 0.19 \\
\hline & DCM & 477 & $\tau=9.99(100 \%)$ & 1.0 & 0.38 \\
\hline & DMSO & 520 & $\tau=16.86(100 \%)$ & 1.2 & 0.41 \\
\hline
\end{tabular}




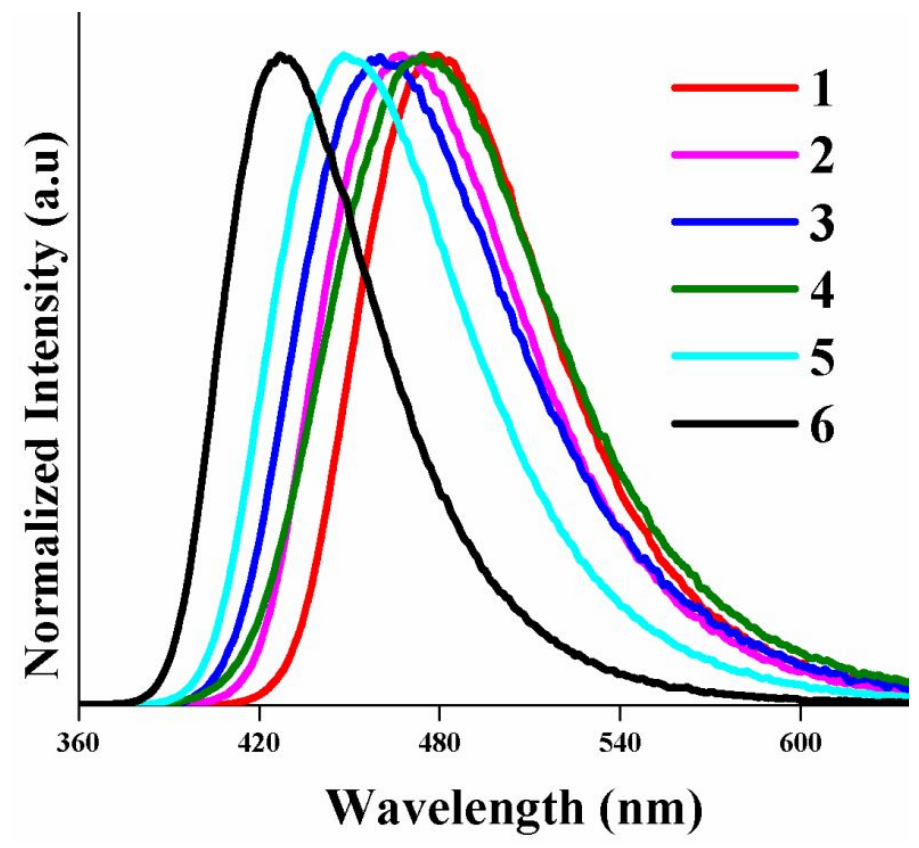

Figure S36. Fluorescence emission spectra of 1-6 in the solid state $\left(\lambda_{\text {ex }}=370 \mathrm{~nm}\right.$ for $\mathbf{1}, \mathbf{2}$ and $\mathbf{3}$ and 330 $\mathrm{nm}$ for 4, 5 and 6; $\boldsymbol{\lambda}_{\mathrm{em}}=470 \mathrm{~nm}(\mathbf{1}), 467 \mathrm{~nm}(\mathbf{2}), 457 \mathrm{~nm}(\mathbf{3}), 474 \mathrm{~nm}(\mathbf{3}) 448 \mathrm{~nm}(\mathbf{5})$ and $\left.427 \mathrm{~nm}(\mathbf{6})\right)$.

Table S5. Photophysical properties of compounds 1-6 in the solid state $\left(\lambda_{\mathrm{ex}}=370 \mathrm{~nm}\right.$ for $\mathbf{1 - 3}$ and $330 \mathrm{~nm}$ for 4-6)

\begin{tabular}{|l|c|l|c|c|c|c|}
\hline & $\lambda_{\mathrm{em}}$ & $\tau(\mathrm{ns})$ & $\chi^{2}$ & $\begin{array}{c}\phi_{\mathrm{F}} \\
(\%)\end{array}$ & $\begin{array}{c}\mathrm{k}_{\mathrm{r}} \\
\left(* 10^{6} \mathrm{~S}^{-1}\right)\end{array}$ & $\begin{array}{c}\mathrm{k}_{\mathrm{nr}} \\
\left(* 10^{6} \mathrm{~S}^{-1}\right)\end{array}$ \\
\hline $\mathbf{1}$ & 470 & $\begin{array}{l}\tau_{1}=0.92(20 \%) \\
\tau_{2}=3.15(60 \%) \\
\tau_{3}=8.34(20 \%)\end{array}$ & 1.08 & 28 & 74.82 & 192.41 \\
\hline $\mathbf{2}$ & 467 & $\begin{array}{l}\tau_{1}=1.01(33 \%) \\
\tau_{2}=3.39(59 \%) \\
\tau_{3}=11.70(8 \%)\end{array}$ & 1.25 & 10 & 30.58 & 275.23 \\
\hline $\mathbf{3}$ & 457 & $\begin{array}{l}\tau_{1}=1.54(87 \%) \\
\tau_{2}=4.05(13 \%)\end{array}$ & 1.26 & 8 & 42.78 & 491.98 \\
\hline $\mathbf{4}$ & 474 & $\begin{array}{l}\tau_{1}=1.78(25 \%) \\
\tau_{2}=6.21(62 \%) \\
\tau_{3}=16.25(13 \%)\end{array}$ & 1.18 & 29 & 45.24 & 110.76 \\
\hline $\mathbf{5}$ & 448 & $\begin{array}{l}\tau_{1}=2.12(28 \%) \\
\tau_{2}=5.42(58 \%) \\
\tau_{3}=13.53(14 \%)\end{array}$ & 1.08 & 19 & 33.75 & 143.87 \\
\hline $\mathbf{6}$ & 427 & $\begin{array}{l}\tau_{1}=1.54(87 \%) \\
\tau_{2}=4.05(13 \%)\end{array}$ & 1.26 & 10 & 53.48 & 481.28 \\
\hline
\end{tabular}



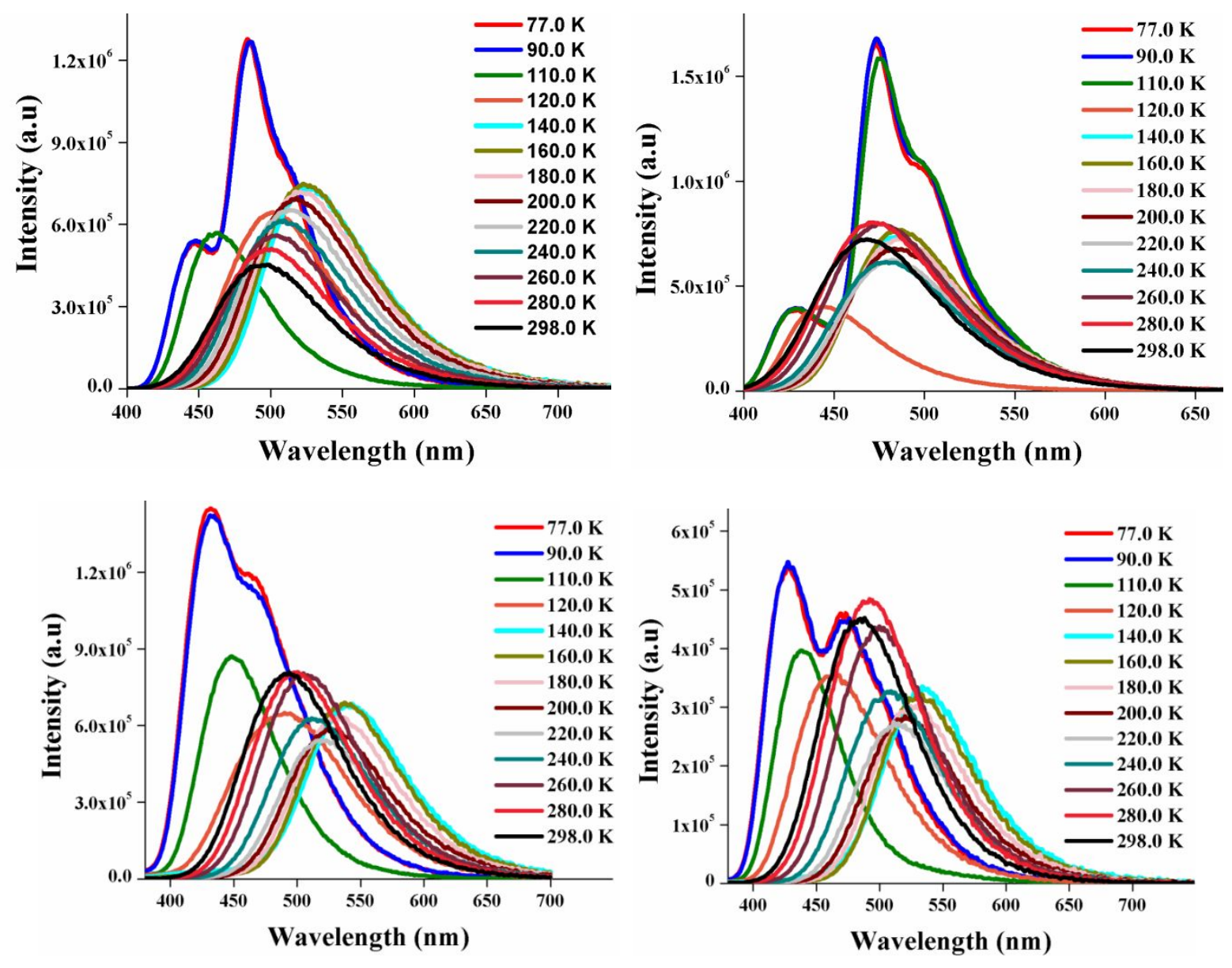

Figure S37. Temperature dependent emission spectra of $\mathbf{2}$ (top left), 3 (top right), 5 (bottom left) and 6 (bottom right) under an inert atmosphere of $\mathrm{N}_{2}$; (solvent: 2 - MeTHF, Concentration $=10 \mu \mathrm{M}, \lambda_{\mathrm{ex}}=370$ $\mathrm{nm}$ for $\mathbf{2}$ and $\mathbf{3}$ and $330 \mathrm{~nm}$ for $\mathbf{5}$ and $\mathbf{6}$ ).
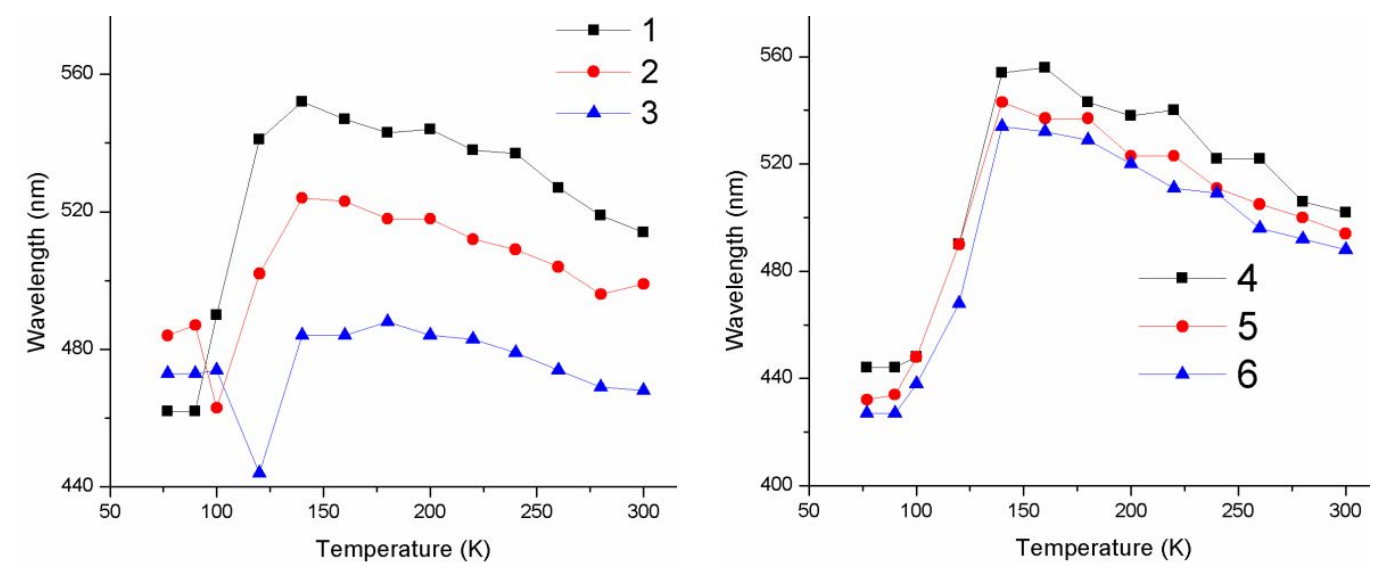

Figure S38. Plot of emission wavelength as a function of temperature in 1-3 (left) and 4-6 (right); (solvent: 2- MeTHF, Concentration $=10 \mu \mathrm{M}, \lambda_{\mathrm{ex}}=370 \mathrm{~nm}$ for 1-3 and $330 \mathrm{~nm}$ for 4-6). 

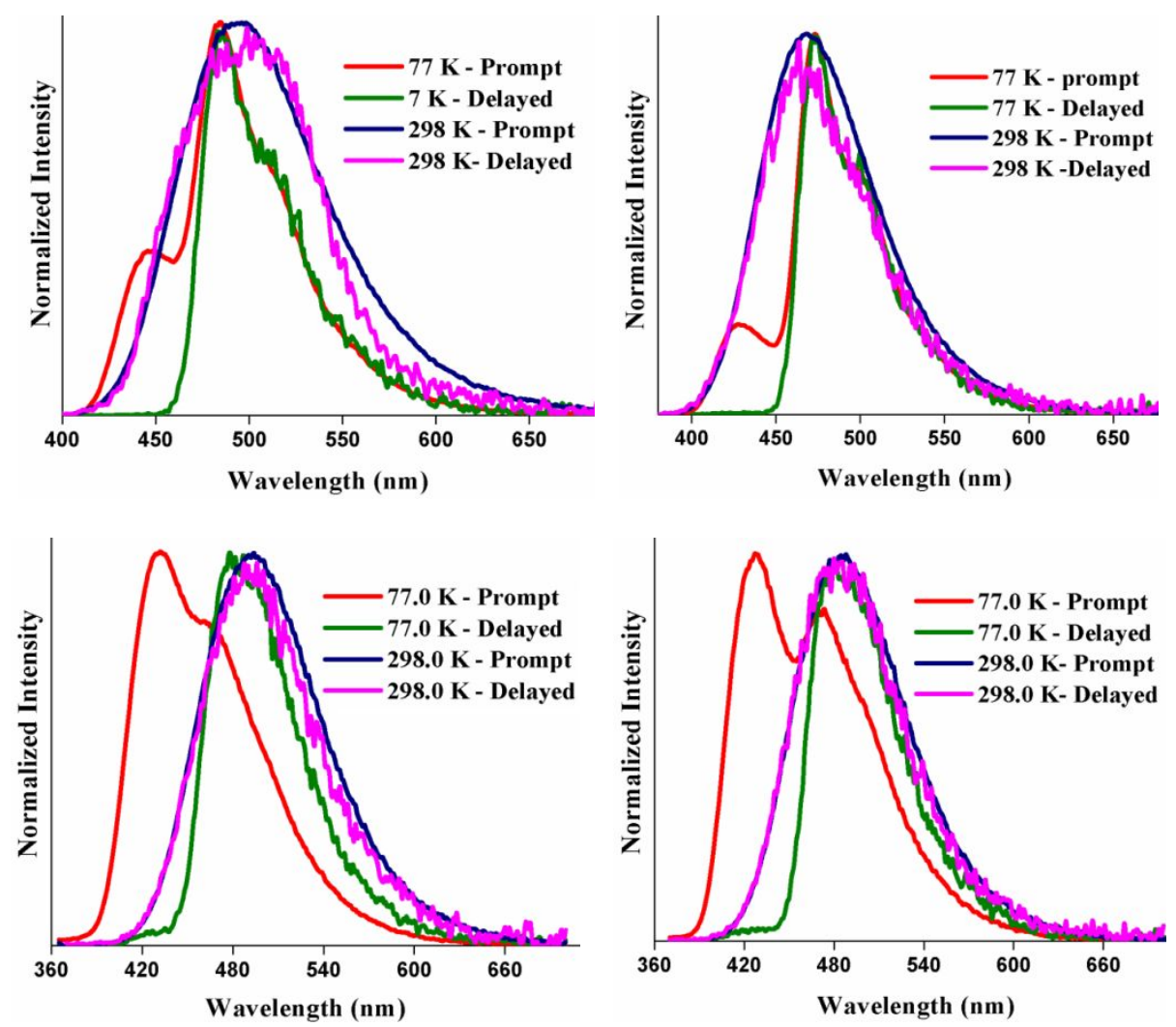

Figure S39. Prompt and delayed emission spectra of $\mathbf{2}$ (top left), 3 (top right), 5 (bottom left) and 6 (bottom right) at $77 \mathrm{~K}$ and $298 \mathrm{~K}$ under an inert atmosphere of $\mathrm{N}_{2}$; (solvent: 2- MeTHF, Concentration = $10 \mu \mathrm{M}, \lambda_{\mathrm{ex}}=370 \mathrm{~nm}$ for $\mathbf{2}$ and $\mathbf{3}$ and $330 \mathrm{~nm}$ for $\mathbf{5}$ and $\left.\mathbf{6}\right)$.

Table S6. Photophysical properties of 1-6 at $298 \mathrm{~K}$ and $77 \mathrm{~K}$ under an inert atmosphere of $\mathrm{N}_{2}$ (solvent: 2MeTHF, Concentration $=10 \mu \mathrm{M}, \lambda_{\mathrm{ex}}=370 \mathrm{~nm}$ for $\mathbf{1 - 3}$ and $330 \mathrm{~nm}$ for 4-6)

\begin{tabular}{|c|c|c|c|c|c|c|c|}
\hline & Temp. & $\begin{array}{c}\lambda_{\mathrm{em}} \\
\text { (Prompt) }\end{array}$ & $\tau_{\text {fluorescence }}(\mathrm{ns})$ & $\chi^{2}$ & $\begin{array}{c}\lambda_{\mathrm{em}} \\
\text { (Delayed) }\end{array}$ & $\tau_{\mathrm{DF} / \text { phosphorescence }}$ & $\chi^{2}$ \\
\hline \multirow[t]{2}{*}{1} & $298 \mathrm{~K}$ & 520 & 12.86 & 1.05 & 520 & $\begin{array}{l}\tau_{1}=4.26 \mu \mathrm{s}(84 \%) \\
\tau_{2}=9.78 \mu \mathrm{s}(16 \%)\end{array}$ & 1.13 \\
\hline & $77 \mathrm{~K}$ & 462 & 13.40 & 1.18 & 503 & $957.74 \mathrm{~ms}$ & 1.18 \\
\hline \multirow[t]{2}{*}{2} & $298 \mathrm{~K}$ & 495 & 7.98 & 1.03 & 495 & $\begin{array}{l}\tau_{1}=5.42 \mu \mathrm{s}(83 \%) \\
\tau_{2}=9.15 \mu \mathrm{s}(17 \%)\end{array}$ & 1.01 \\
\hline & $77 \mathrm{~K}$ & 485 & $\begin{array}{l}\tau_{1}=3.98(82 \%) \\
\tau_{2}=13.60(18 \%)\end{array}$ & 0.98 & 485 & $666.24 \mathrm{~ms}$ & 1.17 \\
\hline \multirow[t]{2}{*}{3} & $298 \mathrm{~K}$ & 465 & 4.88 & 1.15 & 465 & $\begin{array}{l}\tau_{1}=5.00 \mu \mathrm{s}(97 \%) \\
\tau_{2}=16.68 \mu \mathrm{s}(3 \%)\end{array}$ & 0.94 \\
\hline & $77 \mathrm{~K}$ & 473 & 1.96 & 1.12 & 473 & $494.45 \mathrm{~ms}$ & 1.19 \\
\hline \multirow[t]{2}{*}{4} & $298 \mathrm{~K}$ & 500 & 9.77 & 1.10 & 500 & $5.70 \mu \mathrm{s}$ & 1.03 \\
\hline & $77 \mathrm{~K}$ & 444 & $\begin{array}{l}\tau_{1}=6.23(25 \%) \\
\tau_{1}=13.22(75 \%)\end{array}$ & 1.00 & 476 & $0.928 \mathrm{Sec}$ & 1.16 \\
\hline \multirow[t]{2}{*}{5} & $298 \mathrm{~K}$ & 490 & 9.23 & 1.08 & 490 & $5.21 \mu \mathrm{s}$ & 1.01 \\
\hline & $77 \mathrm{~K}$ & 432 & $\begin{array}{l}\tau_{1}=5.08(51 \%) \\
\tau_{2}=10.94(49 \%)\end{array}$ & 1.02 & 490 & $795.23 \mathrm{~ms}$ & 1.20 \\
\hline \multirow[t]{2}{*}{6} & $298 \mathrm{~K}$ & 488 & 8.31 & 1.05 & 488 & $5.19 \mu \mathrm{s}$ & 0.99 \\
\hline & $77 \mathrm{~K}$ & 427 & $\begin{array}{l}\tau_{1}=4.65(70 \%) \\
\tau_{2}=10.40(30 \%)\end{array}$ & 1.13 & 488 & $665.50 \mathrm{~ms}$ & 1.19 \\
\hline
\end{tabular}



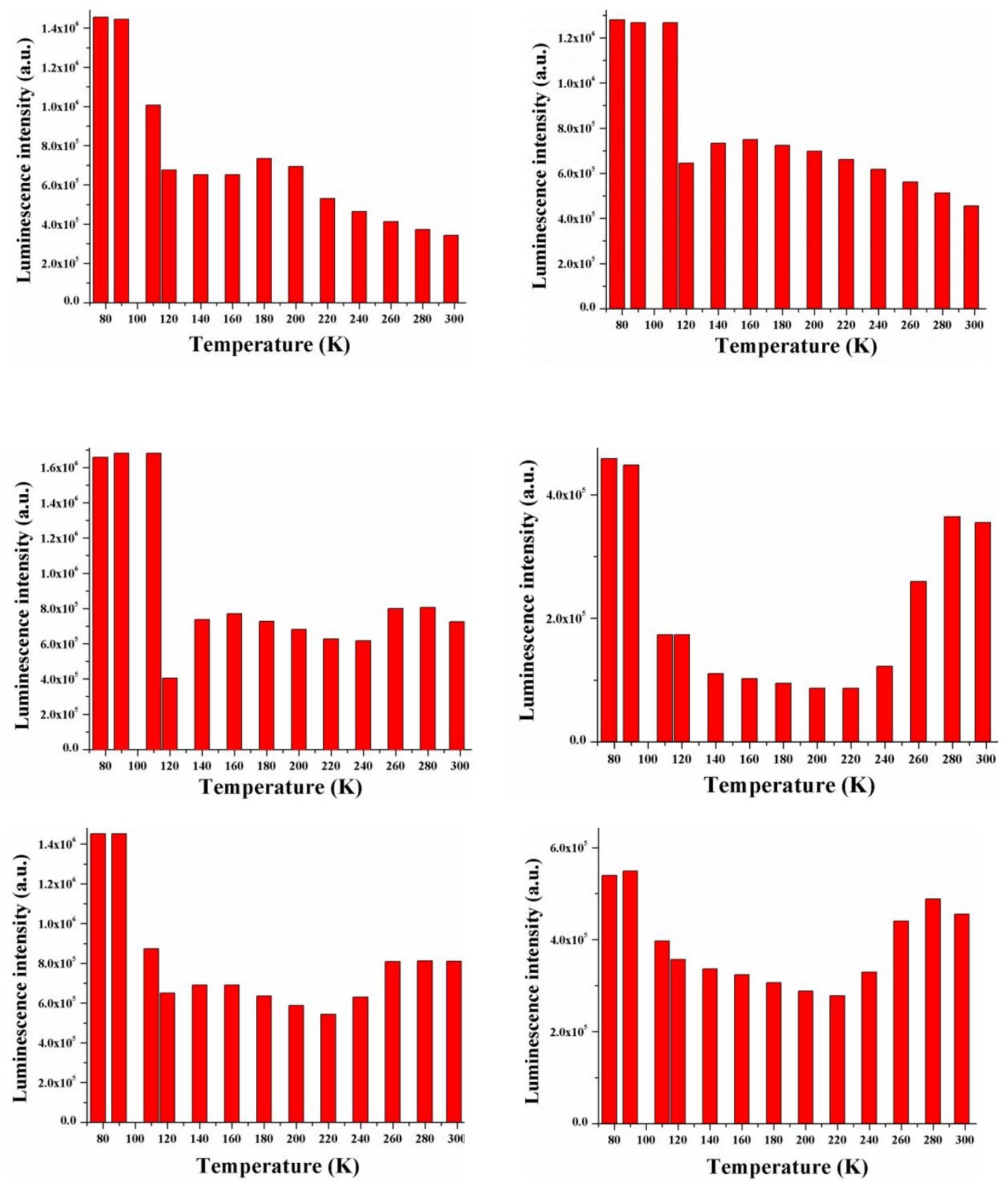

Figure S40. Variation of emission intensity as a function of temperature of $\mathbf{1}$ (top left), $\mathbf{2}$ (top right), $\mathbf{3}$ (middle left), 4 (middle right), 5 (bottom left) and $\mathbf{6}$ (bottom right) under an inert atmosphere of $\mathrm{N}_{2}$; (solvent: 2- MeTHF, Concentration $=10 \mu \mathrm{M}, \lambda_{\mathrm{ex}}=370 \mathrm{~nm}$ for $\mathbf{1}, \mathbf{2}$ and $\mathbf{3}$ and $330 \mathrm{~nm}$ for 4, 5 and $\mathbf{6}$ ). 

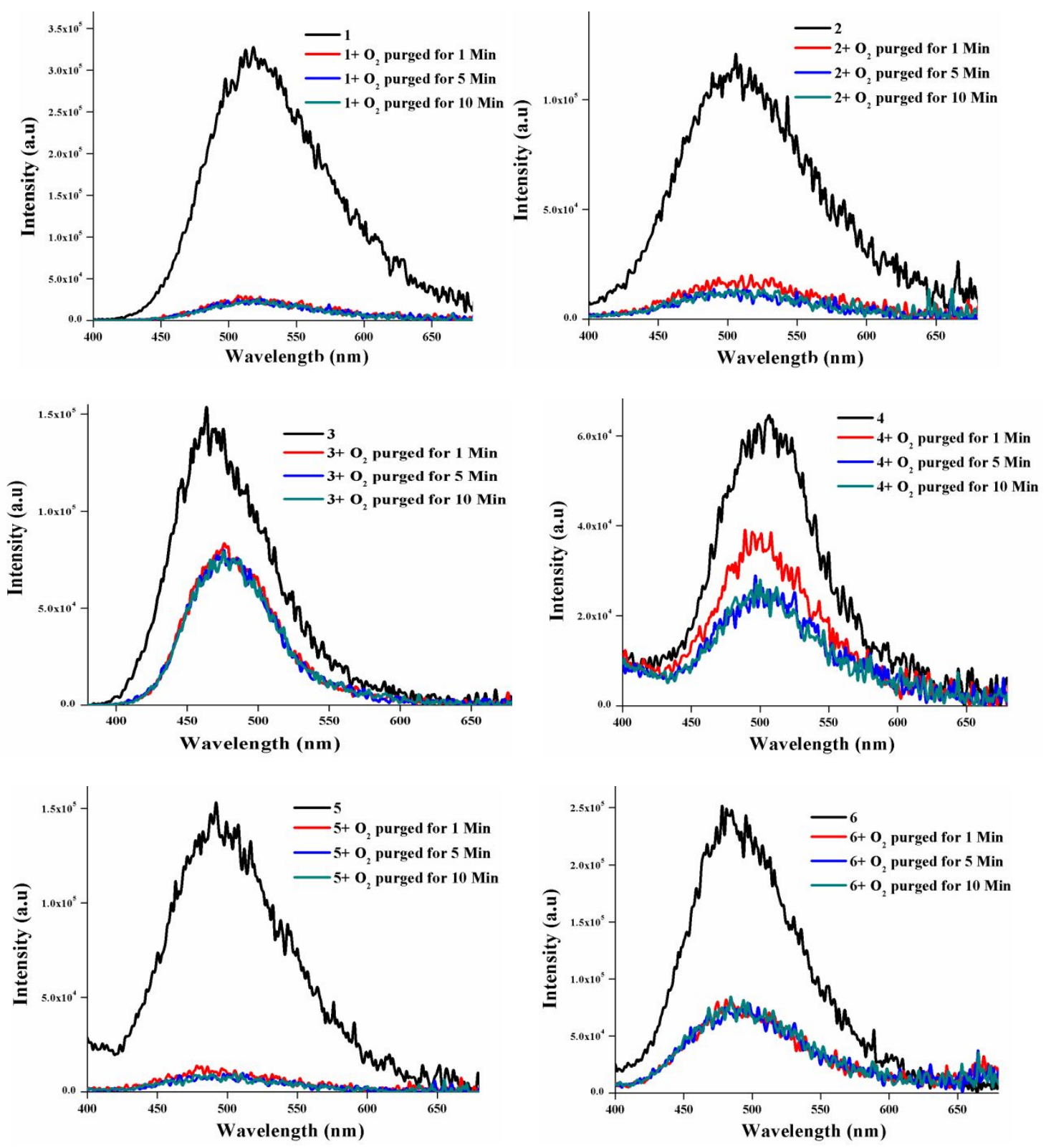

Figure S41. Delayed emission spectra of compounds 1 (top left), 2 (top right), 3 (middle left), 4 (middle right), 5 (bottom left) and $\mathbf{6}$ (bottom right) under an inert atmosphere of $\mathrm{N}_{2}$ and in presence of oxygen at $298 \mathrm{~K}$ (solvent: 2 - MeTHF, Concentration $=10 \mu \mathrm{M}, \lambda_{\mathrm{ex}}=370 \mathrm{~nm}$ for $\mathbf{1 - 3}$ and $330 \mathrm{~nm}$ for $\mathbf{4}-\mathbf{6}$ ). 

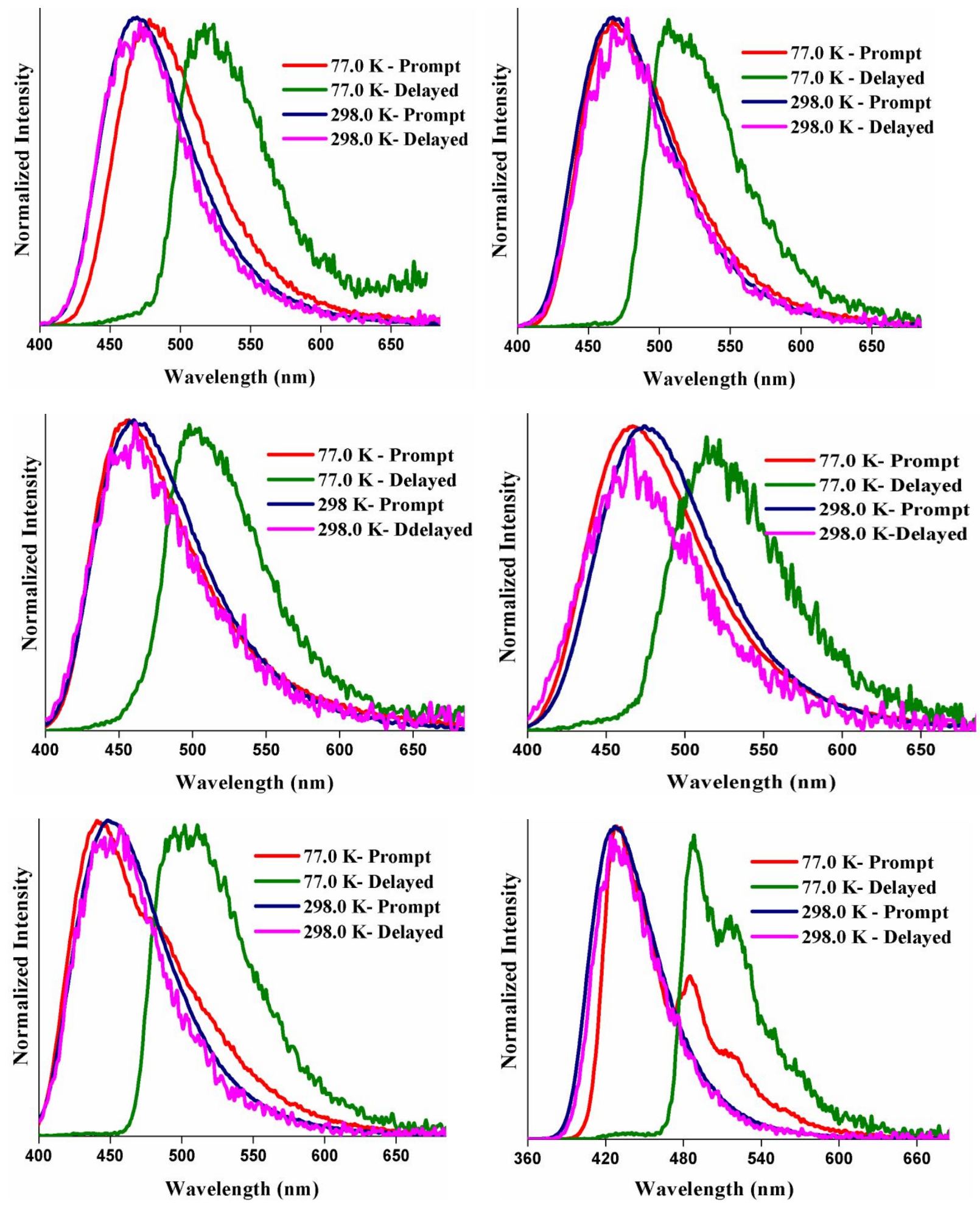

Figure S42. Prompt and delayed emission spectra of compounds 1 (top left), 2 (top right), 3 (middle left), 4 (middle right), 5 (bottom left) and 6 (bottom right) at $77 \mathrm{~K}$ and $298 \mathrm{~K}$ in the solid state under an inert atmosphere of $\mathrm{N}_{2} ;\left(\lambda_{\mathrm{ex}}=370 \mathrm{~nm}\right.$ for $\mathbf{1 , 2}$ and $\mathbf{3}$ and $330 \mathrm{~nm}$ for 4, 5 and $\left.\mathbf{6}\right)$. 

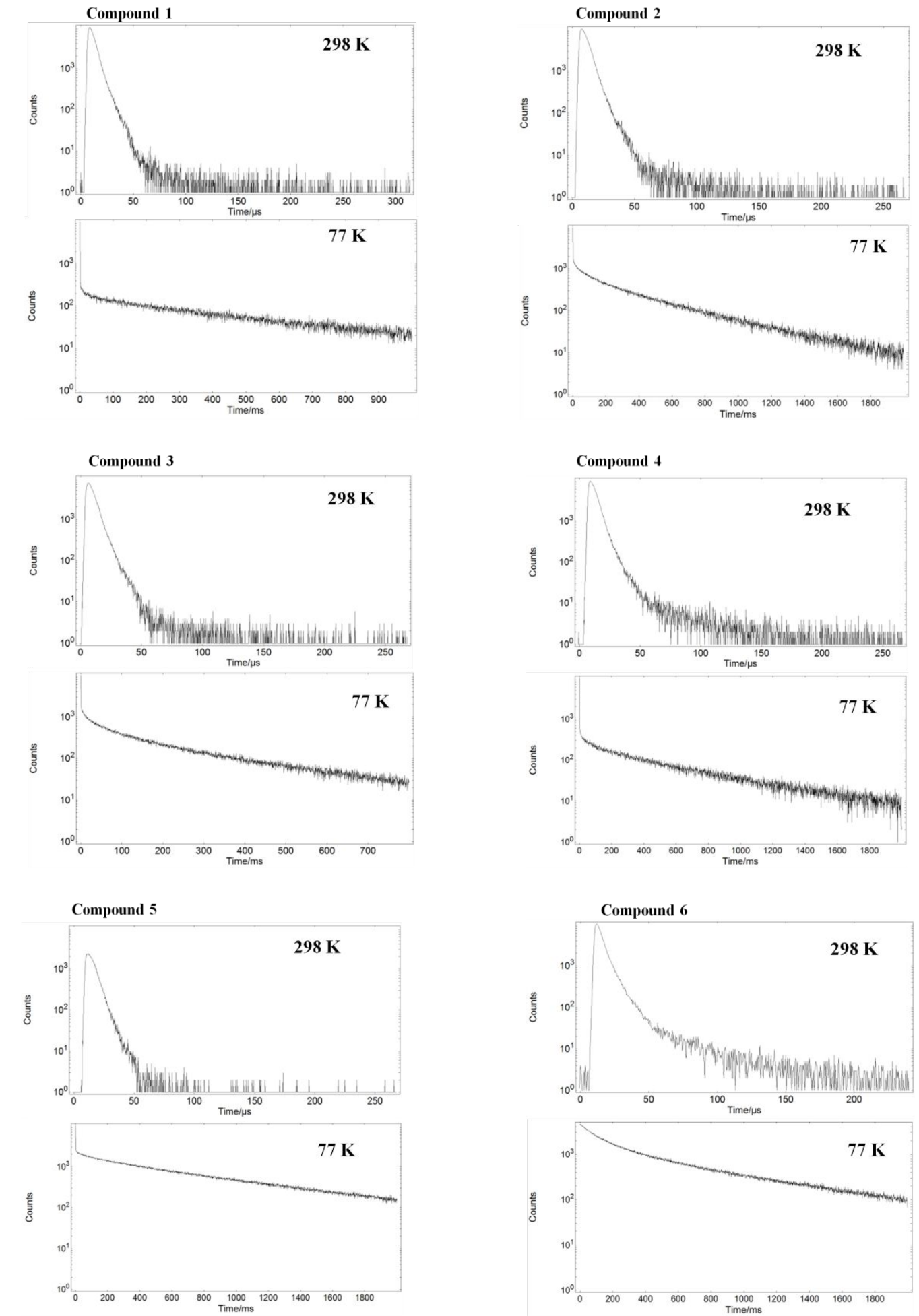

Figure S43. Delayed emission decay curves of 1-6 at $77 \mathrm{~K}$ and $298 \mathrm{~K}$ in the solid state under an inert atmosphere of $\mathrm{N}_{2}\left(\lambda_{\mathrm{ex}}=370 \mathrm{~nm}\right.$ for $\mathbf{1}, 2$ and 3 and $330 \mathrm{~nm}$ for 4, 5 and $\left.\mathbf{6}\right)$. 
Table S7. Photophysical properties of compounds 1-6 at $298 \mathrm{~K}$ and $77 \mathrm{~K}$ in the solid state under inert atmosphere of $\mathrm{N}_{2}\left(\lambda_{\mathrm{ex}}=370 \mathrm{~nm}\right.$ for 1-3 and $330 \mathrm{~nm}$ for 4-6)

\begin{tabular}{|c|c|c|c|c|c|c|c|}
\hline & Temp. & $\begin{array}{c}\lambda_{\mathrm{em}} \\
\text { (Prompt) }\end{array}$ & $\tau_{\text {fluorescence }}(\mathrm{ns})$ & $x^{2}$ & $\begin{array}{c}\lambda_{\mathrm{em}} \\
\text { (Delayed) }\end{array}$ & $\tau_{\mathrm{DF} / \text { phosphorescence }}$ & $\chi^{2}$ \\
\hline \multirow[t]{2}{*}{1} & $298 \mathrm{~K}$ & 470 & $\begin{array}{l}\tau_{1}=0.92(20 \%) \\
\tau_{2}=3.15(60 \%) \\
\tau_{3}=8.34(20 \%)\end{array}$ & 1.08 & 470 & $\begin{array}{l}\tau_{1}=4.71 \mu \mathrm{s}(91 \%) \\
\tau_{2}=10.96 \mu \mathrm{s}(9 \%)\end{array}$ & 1.00 \\
\hline & $77 \mathrm{~K}$ & 478 & $\begin{array}{l}\tau_{1}=2.10(17 \%) \\
\tau_{2}=7.01(61 \%) \\
\tau_{3}=15.63(22 \%)\end{array}$ & 1.18 & 517 & $329.33 \mathrm{~ms}$ & 1.23 \\
\hline \multirow[t]{2}{*}{2} & $298 \mathrm{~K}$ & 467 & $\begin{array}{l}\tau_{1}=1.01(33 \%) \\
\tau_{2}=3.39(59 \%) \\
\tau_{3}=11.70(8 \%)\end{array}$ & 1.25 & 467 & $\begin{array}{l}\tau_{1}=4.67 \mu \mathrm{s}(96 \%) \\
\tau_{1}=14.25 \mu \mathrm{s}(4 \%)\end{array}$ & 1.08 \\
\hline & $77 \mathrm{~K}$ & 467 & $\begin{array}{l}\tau_{1}=1.46(21 \%) \\
\tau_{2}=4.30(57 \%) \\
\tau_{3}=10.45(22 \%)\end{array}$ & 1.23 & 506 & $\begin{array}{l}\tau_{1}=74.25 \mathrm{~ms}(16 \%) \\
\tau_{2}=402.63 \mathrm{~ms}(84 \%)\end{array}$ & 1.16 \\
\hline \multirow[t]{2}{*}{3} & $298 \mathrm{~K}$ & 457 & $\begin{array}{l}\tau_{1}=0.62(56 \%) \\
\tau_{2}=2.23(39 \%) \\
\tau_{3}=7.62(5 \%)\end{array}$ & 1.11 & 457 & $\begin{array}{l}\tau_{1}=5.05 \mu \mathrm{s}(97 \%) \\
\tau_{2}=16.37 \mu \mathrm{s}(3 \%)\end{array}$ & 1.01 \\
\hline & $77 \mathrm{~K}$ & 457 & $\begin{array}{l}\tau_{1}=0.87(40 \%) \\
\tau_{2}=2.72(54 \%) \\
\tau_{3}=8.60(6 \%)\end{array}$ & 1.22 & 498 & $\begin{array}{l}\tau_{1}=31.64 \mathrm{~ms}(16 \%) \\
\tau_{2}=200.49 \mathrm{~ms}(84 \%)\end{array}$ & 1.22 \\
\hline \multirow[t]{2}{*}{4} & $298 \mathrm{~K}$ & 474 & $\begin{array}{l}\tau_{1}=1.78(25 \%) \\
\tau_{2}=6.21(62 \%) \\
\tau_{3}=16.25(13 \%)\end{array}$ & 1.18 & 474 & $\begin{array}{l}\tau_{1}=4.68 \mu \mathrm{s}(96 \%) \\
\tau_{2}=23.31 \mu \mathrm{s}(4 \%)\end{array}$ & 1.11 \\
\hline & $77 \mathrm{~K}$ & 467 & $\begin{array}{l}\tau_{1}=1.95(22 \%) \\
\tau_{2}=6.09(59 \%) \\
\tau_{3}=14.48(9 \%)\end{array}$ & 1.09 & 522 & $\begin{array}{l}\tau_{1}=52.58 \mathrm{~ms}(8 \%) \\
\tau_{2}=453.03 \mathrm{~ms}(92 \%)\end{array}$ & 1.17 \\
\hline \multirow[t]{2}{*}{5} & $298 \mathrm{~K}$ & 448 & $\begin{array}{l}\tau_{1}=2.12(28 \%) \\
\tau_{2}=5.42(58 \%) \\
\tau_{3}=13.53(14 \%)\end{array}$ & 1.08 & 448 & $5.57 \mu \mathrm{s}$ & 0.99 \\
\hline & $77 \mathrm{~K}$ & 441 & $\begin{array}{l}\tau_{1}=2.32(23 \%) \\
\tau_{2}=6.30(65 \%) \\
\tau_{3}=14.62(12 \%)\end{array}$ & 1.12 & 502 & $\begin{array}{l}\tau_{1}=84.23 \mathrm{~ms}(4 \%) \\
\tau_{2}=723.13 \mathrm{~ms}(96 \%)\end{array}$ & 1.12 \\
\hline \multirow[t]{3}{*}{6} & $298 \mathrm{~K}$ & 427 & $\begin{array}{l}\tau_{1=1.54(87 \%)} \\
\tau_{2}=4.05(13 \%)\end{array}$ & 1.26 & 427 & $\begin{array}{l}\tau_{1}=5.03 \mu \mathrm{s}(84 \%) \\
\tau_{2}=23.45 \mu \mathrm{s}(16 \%)\end{array}$ & 1.23 \\
\hline & \multirow[t]{2}{*}{$77 \mathrm{~K}$} & \multirow[t]{2}{*}{427} & \multirow[t]{2}{*}{$\begin{array}{l}\tau_{1}=2.50(68 \%) \\
\tau_{2}=35.20(32 \%)\end{array}$} & \multirow[t]{2}{*}{1.13} & 488 & $\begin{array}{l}\tau_{1}=117.68 \mathrm{~ms}(30 \%) \\
\tau_{2}=567.95 \mathrm{~ms}(70 \%)\end{array}$ & 1.22 \\
\hline & & & & & 515 & $\begin{array}{l}\tau_{1}=112.26 \mathrm{~ms}(25 \%) \\
\tau_{2}=580.90 \mathrm{~ms}(75 \%)\end{array}$ & 1.24 \\
\hline
\end{tabular}



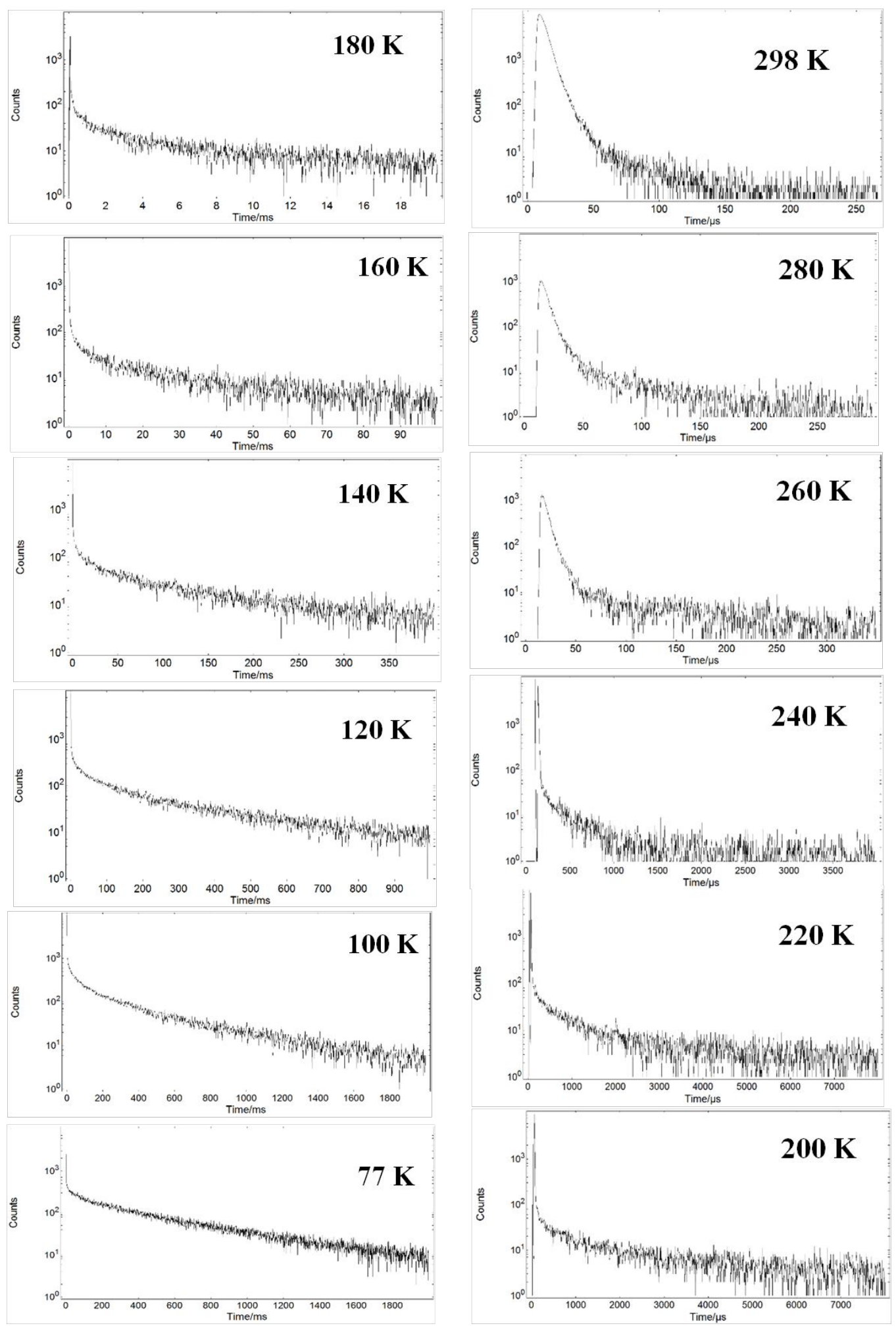

Figure S44. Delayed emission decay curves of compound $\mathbf{4}$ at different temperatures in the solid state an under inert atmosphere of $\mathrm{N}_{2}\left(\lambda_{\mathrm{ex}}=330 \mathrm{~nm}\right)$. 

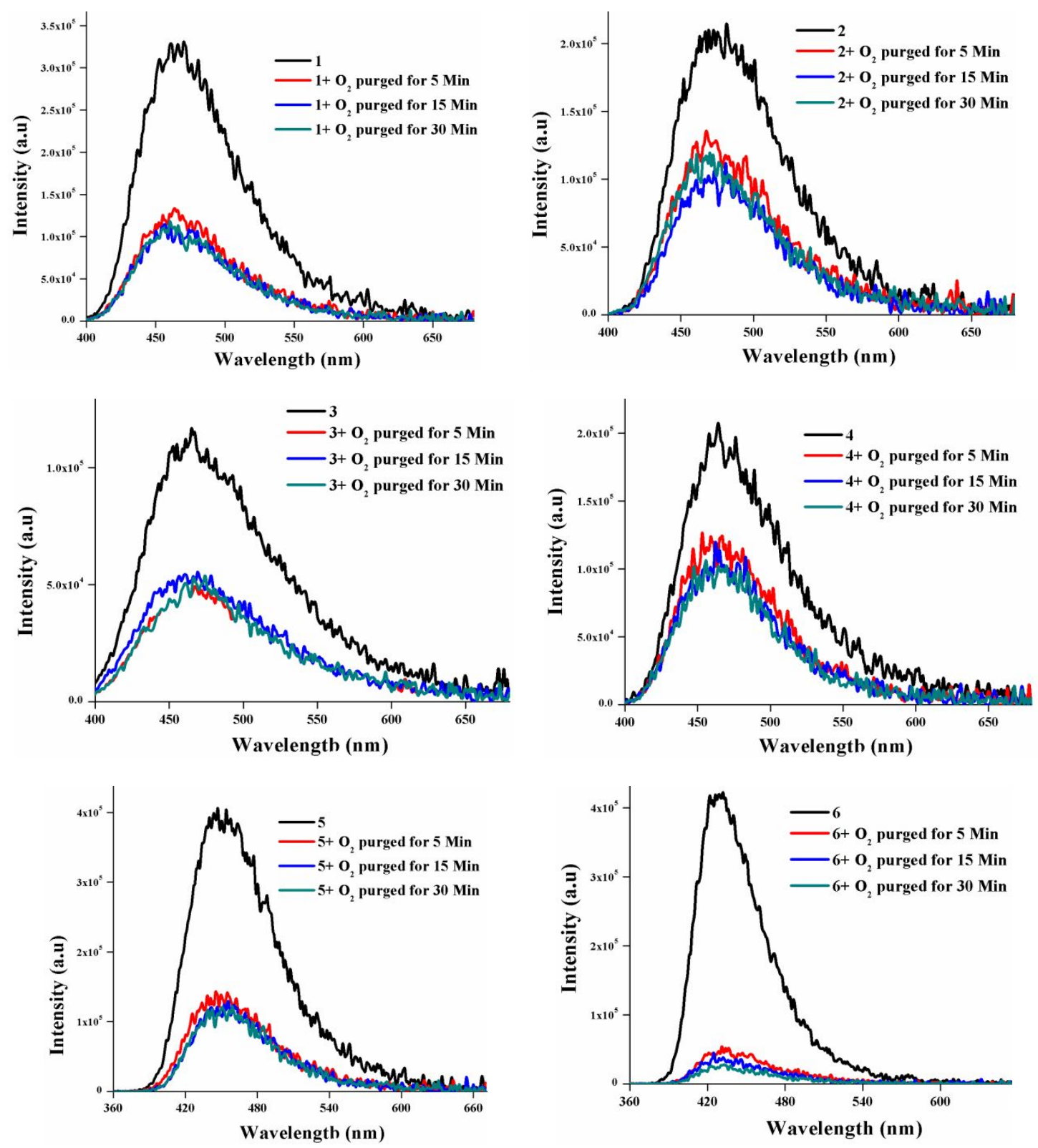

Figure S45. Delayed emission spectra of compounds 1 (top left), 2 (top right), 3 (middle left), 4 (middle right), 5 (bottom left) and $\mathbf{6}$ (bottom right) under an inert atmosphere of $\mathrm{N}_{2}$ and in presence of oxygen at $298 \mathrm{~K}$ in the solid state $\left(\lambda_{\mathrm{ex}}=370 \mathrm{~nm}\right.$ for $\mathbf{1 - 3}$ and $330 \mathrm{~nm}$ for $\left.\mathbf{4}-\mathbf{6}\right)$. 

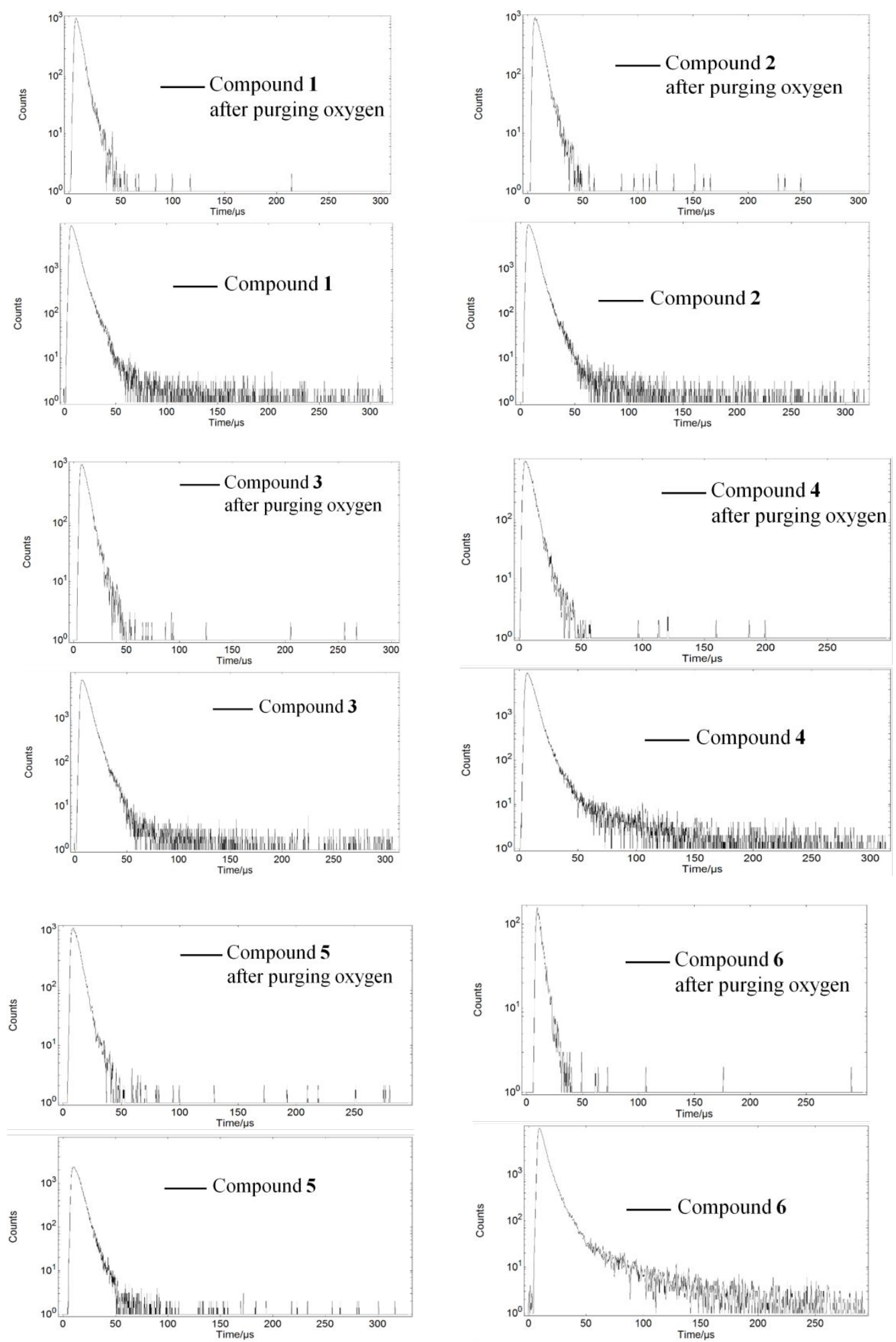

Figure S46. Delayed emission decay profiles of compounds 1-6 at different temperatures in the solid state under an inert atmosphere of $\mathrm{N}_{2}$ and in presence of oxygen at $298 \mathrm{~K}$ in the solid state $\left(\lambda_{\mathrm{ex}}=370 \mathrm{~nm}\right.$ for 13 and $330 \mathrm{~nm}$ for $4-6)$. 

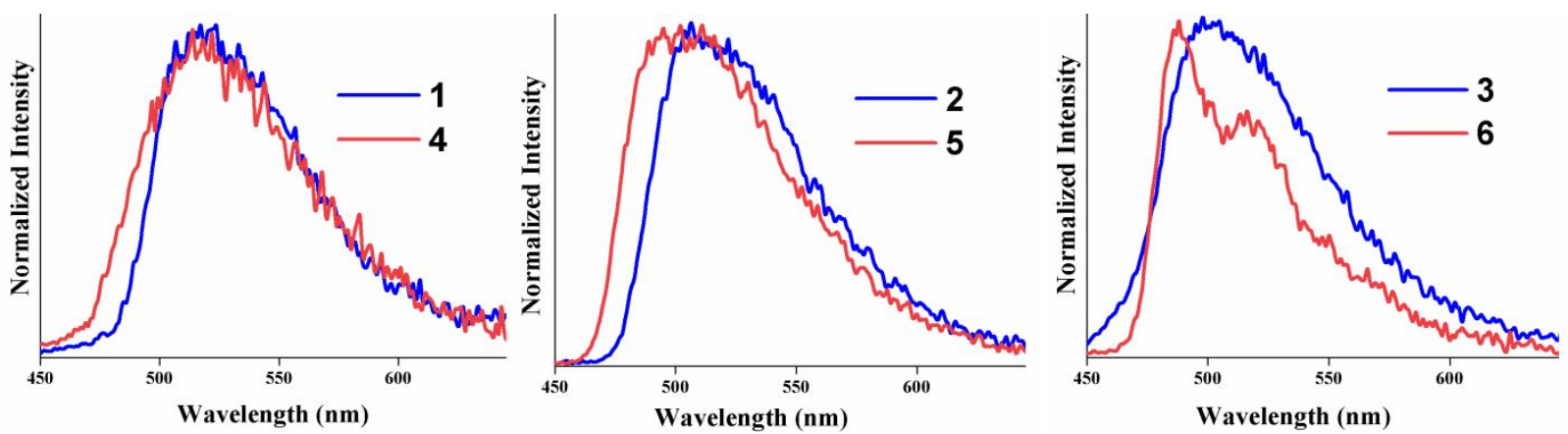

Figure S47. Comparison of phosphorescence emission spectra of $-\mathrm{NH}_{2}$ and corresponding $-\mathrm{NMe}_{2}$ derivatives in the solid state at $77 \mathrm{~K}, \mathbf{1}$ and $\mathbf{4}$ (top left), $\mathbf{2}$ and $\mathbf{5}$ (middle) and $\mathbf{3}$ and $\mathbf{6}$ (right) under an inert atmosphere of $\mathrm{N}_{2} ;\left(\lambda_{\mathrm{ex}}=370 \mathrm{~nm}\right.$ for $\mathbf{1}, \mathbf{2}$ and $\mathbf{3}$ and $330 \mathrm{~nm}$ for $\mathbf{4 , 5}$ and $\left.\mathbf{6}\right)$.

Table S8. Singlet $\left(\mathrm{S}_{1}\right)$ and triplet $\left(\mathrm{T}_{1}\right)$ energies and the energy gap $\left(\Delta \mathrm{E}_{\mathrm{ST}}\right)$ between them determined from (i) experimental peak positions of prompt fluorescence and phosphorescence $(77 \mathrm{~K})$ in solution and solid thin film state and iii) theoretical calculations (DFT/TD-DFT/B3LYP/6-31G (d)).

\begin{tabular}{|c|c|c|c|c|c|c|c|c|c|c|}
\hline & \multicolumn{6}{|c|}{ Experiment } & \multirow{2}{*}{\multicolumn{4}{|c|}{ Theory (vacuum) }} \\
\hline & \multicolumn{3}{|c|}{ Solution } & \multicolumn{3}{|c|}{ Solid state } & & & & \\
\hline & $S_{1}(e V)$ & $T_{1}(e V)$ & $\Delta E_{S T}(e V)$ & $S_{1}(e V)$ & $T_{1}(e V)$ & $\begin{array}{c}\Delta \mathbf{E}_{\text {ST }} \\
(\mathrm{eV})\end{array}$ & $S_{1}(e V)$ & $T_{1}(e V)$ & $\Delta E_{S T}(e V)$ & $f$ \\
\hline 1 & 2.6894 & 2.4796 & 0.209 & 2.5992 & 2.3843 & 0.2149 & 3.3692 & 2.8084 & 0.5608 & 0.159 \\
\hline 2 & 2.7737 & 2.5302 & 0.243 & 2.6492 & 2.4310 & 0.2182 & 3.3254 & 2.8478 & 0.4776 & 0.183 \\
\hline 3 & 2.8968 & 2.6267 & 0.270 & 2.7070 & 2.4796 & 0.2274 & 3.4082 & 2.9086 & 0.4996 & 0.184 \\
\hline 4 & 2.7924 & 2.6435 & 0.148 & 2.6549 & 2.3843 & 0.2706 & 3.3621 & 3.0187 & 0.3434 & 0.055 \\
\hline 5 & 2.8700 & 2.5938 & 0.276 & 2.8050 & 2.4600 & 0.3450 & 3.3179 & 2.9964 & 0.3215 & 0.089 \\
\hline 6 & 2.8833 & 2.5937 & 0.289 & 2.8968 & 2.5406 & 0.3562 & 3.2873 & 2.9687 & 0.3186 & 0.091 \\
\hline
\end{tabular}




\section{Theoretical Calculations}

Table S9. Summary of the excited state electronic transitions obtained from the TD-DFT calculations.

\begin{tabular}{|c|c|c|c|c|c|}
\hline Compound & Excited State & $\mathrm{E} / \mathrm{eV}$ & $\mathrm{E} / \mathrm{nm}$ & $\bar{f}$ & $\begin{array}{l}\text { Dominant transitions } \\
\text { (percent contribution) }\end{array}$ \\
\hline \multirow{3}{*}{1} & 1 & 3.37 & 368 & 0.1597 & HOMO ->LUMO (95\%) \\
\hline & 2 & 3.80 & 326 & 0.0083 & HOMO-1 ->LUMO (98\%) \\
\hline & 3 & 3.83 & 324 & 0.0121 & HOMO-2 ->LUMO (97\%) \\
\hline \multirow[b]{2}{*}{2} & 1 & 3.32 & 373 & 0.1832 & HOMO ->LUMO (98\%) \\
\hline & 2 & 3.50 & 354 & 0.0968 & HOMO-1 ->LUMO (95\%) \\
\hline \multirow[b]{2}{*}{3} & 1 & 3.32 & 373 & 0.1834 & HOMO ->LUMO (98\%) \\
\hline & 2 & 3.43 & 362 & 0.2016 & HOMO-1 ->LUMO (95\%) \\
\hline \multirow{3}{*}{4} & 1 & 3.36 & 369 & 0.0548 & HOMO ->LUMO (95\%) \\
\hline & 2 & 3.70 & 334 & 0.0122 & HOMO-1 ->LUMO (97\%) \\
\hline & 3 & 3.72 & 332 & 0.0109 & HOMO-2 ->LUMO (98\%) \\
\hline \multirow{4}{*}{5} & 1 & 3.32 & 374 & 0.0895 & HOMO ->LUMO (95\%) \\
\hline & 2 & 3.36 & 368 & 0.0217 & HOMO-1 ->LUMO (95\%) \\
\hline & 3 & 3.68 & 336 & 0.0097 & HOMO-2 ->LUMO (97\%) \\
\hline & 4 & 3.68 & 336 & 0.0140 & HOMO-2 ->LUMO (97\%) \\
\hline \multirow{4}{*}{6} & 1 & 3.28 & 377 & 0.0912 & HOMO ->LUMO (95\%) \\
\hline & 2 & 3.32 & 373 & 0.0787 & $\begin{array}{l}\text { HOMO-2 ->LUMO (95\%) } \\
\text { HOMO-1 ->LUMO (18\%) }\end{array}$ \\
\hline & 3 & 3.35 & 369 & 0.0010 & $\begin{array}{c}\text { HOMO-2 ->LUMO (18\%) } \\
\text { HOMO-1 ->LUMO (72\%) } \\
\text { HOMO ->LUMO (7\%) }\end{array}$ \\
\hline & 4 & 3.64 & 339 & 0.0114 & HOMO-2 ->LUMO (95\%) \\
\hline
\end{tabular}




\section{References}

1. Dawson, W. R.; Windsor, M. W., Fluorescence yields of aromatic compounds. J. Phys. Chem. 1968, 72 (9), 3251-3260.

2. a) SAINT-NT, Version 6.04; Bruker AXS: Madison, WI, 2001; b) SHELXTL-NT, Version 6.10; Bruker AXS: Madison, WI, 2000

3. a) Becke, A. D., Density-functional thermochemistry. III. The role of exact exchange. J. Chem. Phys 1993, 98 (7), 5648-5652; b) Frisch, M. J.; Trucks, G. W.; Schlegel, H. B.; Scuseria, G. E.; Robb, M. A.; Cheeseman, J. R.; Scalmani, G.; Barone, V.; Mennucci, B.; Petersson, G. A.; Nakatsuji, H.; Caricato, M.; X. Li., Hratchian H. P, Izmaylov A. F., Bloino J., Zheng. G., Sonnenberg. J. L., Hada. M., Ehara. M., Toyota. K., Fukuda, R.; Hasegawa, J.; Ishida, M.; Nakajima, T.; Honda, Y.; Kitao, O.; Nakai, H.; Vreven, T.; Montgomery, J. A.; Jr., Peralta, J. E.; Ogliaro, F.; Bearpark, M.; Heyd, J. J.; Brothers, E.; Kudin, K. N.; Staroverov, V. N.; Kobayashi, R.; Normand, J.; Raghavachari, K.; Rendell, A.; Burant, J. C.; Iyengar, S. S.; Tomasi, J.; Cossi, M.; Rega, N.; Millam, J. M.; Klene, M.; Knox, J. E.; Cross, J. B.; Bakken, V.; Adamo, C.; Jaramillo, J.; Gomperts, R.; Stratmann, R. E.; Yazyev, O.; Austin, A. J.; Cammi, R.; Pomelli, C.; Ochterski, J. W.; Martin, R. L.; Morokuma, K.; Zakrzewski, V. G.; Voth, G. A.; Salvador, P.; Dannenberg, J. J.; Dapprich, S.; Daniels O. Farkas, A. D.; Foresman, J. B.; Ortiz, J. V.; Cioslowski, J.; Fox, D. J. Gaussian 09, Revision A.02, Gaussian, Inc., Wallingford, CT, 2009; c) Becke, A. D., Densityfunctional exchange-energy approximation with correct asymptotic behavior. Phys. Rev. A. 1988, 38 (6), 3098-3100; d) Lee, C.; Yang, W.; Parr, R. G., Development of the Colle-Salvetti correlation-energy formula into a functional of the electron density. Phys. Rev. B. 1988, 37 (2), 785-789.

4. Liu, Y.; Xu, X.; Zheng, F.; Cui, Y., Chiral Octupolar Metal-Organoboron NLO Frameworks with $(14,3)$ Topology. Angew. Chem. Int. Ed. 2008, 47 (24), 4538-4541. 